\title{
PROPUESTAS DE ENSEÑANZA DE LA FORMULACIÓN QUÍMICA EN MANUALES DE 3 Y 4 DE ESO
}

Máster Universitario de Profesorado de Educación Secundaria Obligatoria, Bachillerato, Formación Profesional y Enseñanza de Idiomas

Trabajo Fin de Máster - Junio de 2013

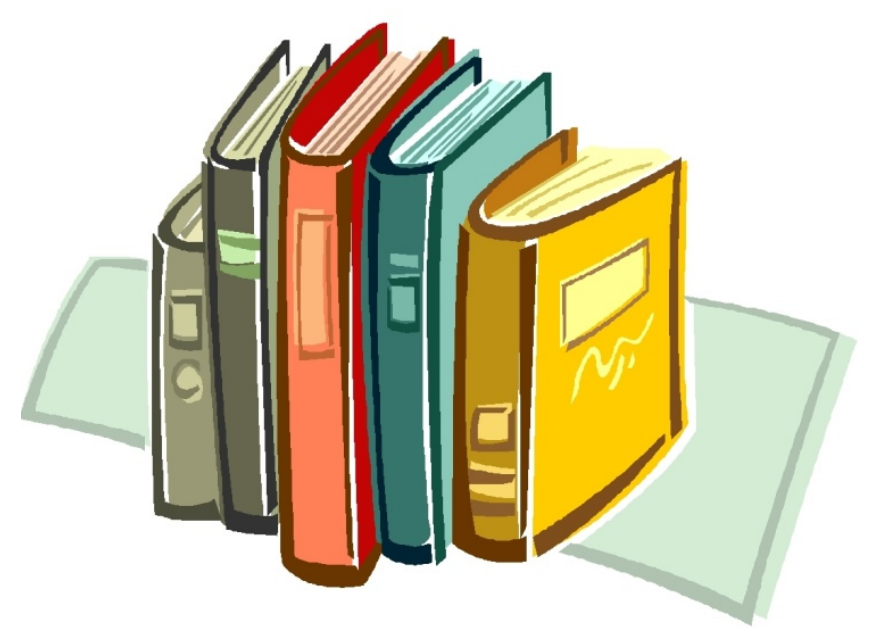

Alumno: Almudena Linares Casado

Profesor Supervisor: Manuel Fernández González 


\section{Formulación}

\section{Química}

\section{Inorgánica}

\section{Resumen:}

Al inicio de cualquier curso elemental de química encontramos, entre otros contenidos, la formulación y nomenclatura de los compuestos químicos inorgánicos.

Aprender a formular es un contenido básico para introducir al alumno en el mundo de la química y, por tanto, prepararlo para comprender desarrollos posteriores de la disciplina. Así se consigue la familiarización con el lenguaje científico de esta rama de la ciencia y conocemos, además, el significado que encierra.

En nuestro trabajo hemos analizado la exposición y el tratamiento de la temática indicada en los libros de texto de $3^{\circ}$ y $4^{\circ}$ ESO, ya que son en estos cursos donde se inicia el estudio de la formulación química. Hemos tenido en cuenta lo descrito en el Decreto de Enseñanzas Mínimas de esta etapa.

El estudio de los manuales ha arrojado unos resultados que hemos analizado y discutido, para posteriormente extraer las correspondientes conclusiones. 


\section{ÍNDICE}

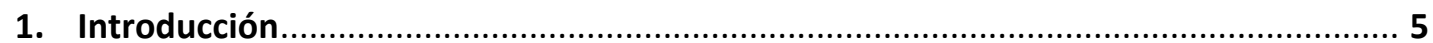

1.1 La importancia de la formulación en la enseñanza de la química. Dificultades de enseñanza y aprendizaje ............................................................................................. 5

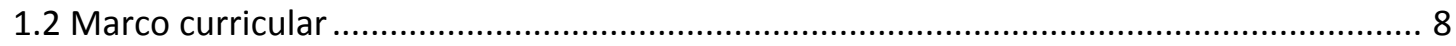

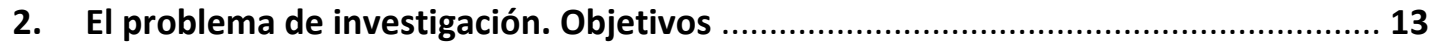

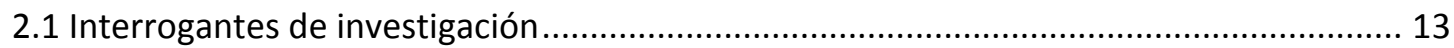

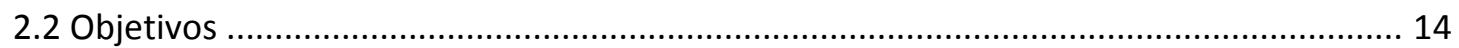

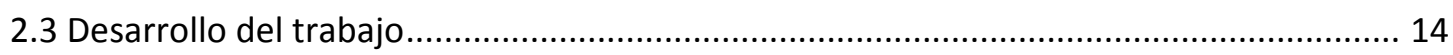

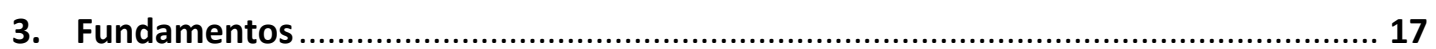

3.1 Nomenclatura y formulación. Conceptos de base ......................................................... 17

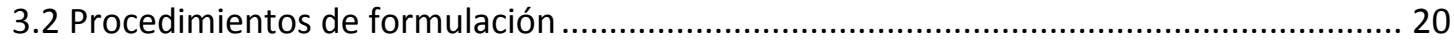

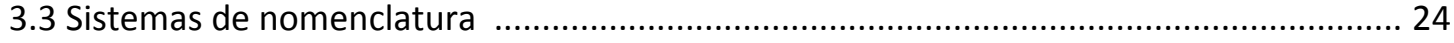

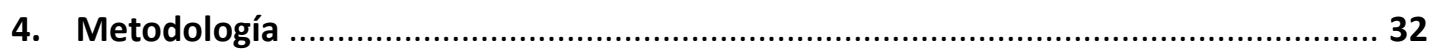

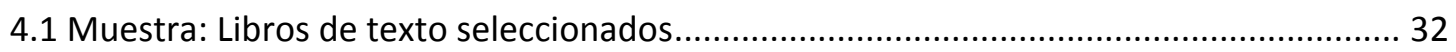

4.2 Tópicos a considerar en el análisis ................................................................................... 33

4.3 Protocolo de análisis de libros de texto ............................................................................... 36

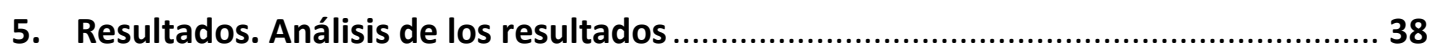

5.1 Características de formulación química en los manuales ............................................... 38

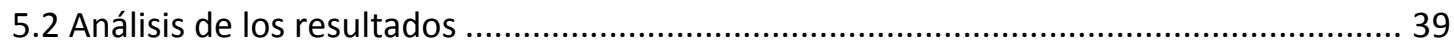

5.3 Discusión de resultados .................................................................................................... 42

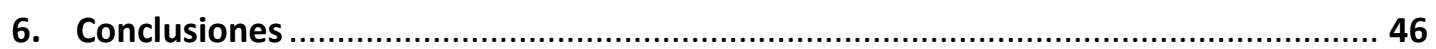

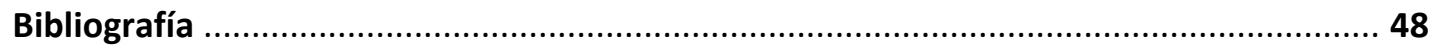

Anexos …

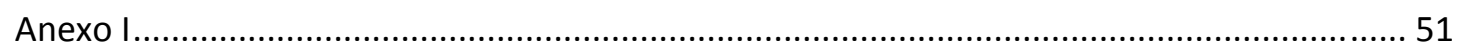

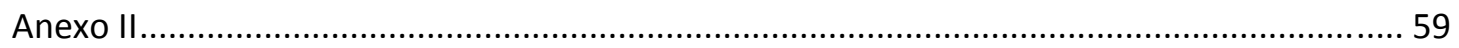

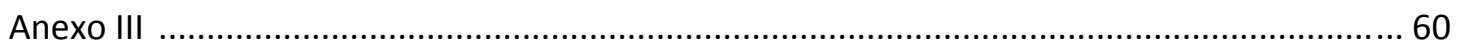

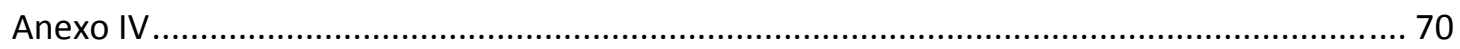




\section{INTRODUCCIÓN}

\subsection{LA IMPORTANCIA DE LA FORMULACIÓN EN LA ENSEÑANZA DE LA QUÍMICA. DIFICULTADES DE ENSEÑANZA Y APRENDIZAJE}

"Dime y lo olvido, enséñame y lo recuerdo, involúcrame y lo aprendo" (Benjamín Franklin, 1706-1790).

La enseñanza de las ciencias es una pieza importante dentro del panorama educativo. La formación del estudiante debe comprender unos aspectos científicos mínimos para poder prepararlos para la vida en una sociedad altamente tecnificada, tal como nos indica el Real Decreto de Enseñanzas Mínimas, donde la educación científica está englobada dentro de la competencia en el conocimiento y la interacción con el mundo físico, una de las ocho competencias básicas (Ministerio, 2006, p. 692).

Las ciencias experimentales, por tanto, tienen una finalidad formativa. Con ellas se pretende desarrollar en el alumno actitudes científicas como son la observación y reflexión de los fenómenos que ocurren a su alrededor, y el intento de encontrar explicación válida a que ocurra una cosa y no otra diferente (Ministerio, 2006, p. 690).

Pero la enseñanza habitual de las ciencias sigue centrada en aspectos muy conceptuales con pocas alusiones a otros que, según las investigaciones en didáctica, provocarían un aumento de la motivación y el interés en su estudio. Este tipo de contenidos aun no ha sido implementado adecuadamente en el currículum ni en la práctica docente. Posiblemente esto sea así porque la enseñanza de las ciencias suele olvidar que su principal finalidad es "preparar a los futuros científicos/ingenieros y a los ciudadanos conjuntamente para participar en una sociedad cada vez más moldeada por la investigación y el desarrollo en ciencia y tecnología" (Solbes, Montserrat y Furió, 2007, p. 97).

Los profesores siguen, pues, en su mayoría la metodología tradicional, centrada en la propia disciplina (Jiménez-Aleixandre, 2000), sin dar el paso a hacer las clases más atractivas. De manera natural, los jóvenes presentan curiosidad hacia la ciencia. Pero esta curiosidad se ve anulada cuando se tratan los temas en las aulas siguiendo la metodología tradicional (Rocard, Csermely, Jorde, Lenzen, WalwergHenriksson y Hemmo, 2008, p. 108).

Centrándonos en nuestra materia cabe decir que, en casi todo el mundo, la enseñanza de la química adquiere un perfil "químicamente puro", lo cual no 
favorece ni ayuda a formar en el alumno una actitud científica frente a la vida, (Chamizo, Nieto y Sosa, 2004). Los estudiantes tienen una visión y un concepto muy negativo sobre la química, piensan que es difícil y poco interesante (Solbes, Montserrat y Furió, 2007). La falta de interés se deriva de la ausencia de conexiones con la realidad, ya que la enseñanza habitual, excesivamente teórica, ignora el valor educativo de lo contextual (Parchmann, 2011). Tratando de corregir esta situación recientemente los países más avanzados han implantado en sus planes de estudio una asignatura común para todos los alumnos y que en España se llama "Ciencias para el mundo contemporáneo". Presta especial atención a contenidos contextuales y CTS mediante una metodología de investigación (Fernández-González, 2008).

Las últimas leyes de educación (LOGSE, LOCE, LOE) no conceden demasiado desarrollo temporal a las asignaturas de ciencias, que sólo son obligatorias hasta 3ํ de ESO, con tan sólo dos horas lectivas a la semana de Física y Química. Además, en 4ํ de ESO la Física y Química debe competir con otras optativas más atractivas como Plástica, Tecnología y Música.

Otro factor que puede tener que ver en este desinterés hacia la química es el tema de los libros de texto. Éstos tienen mucha responsabilidad en el proceso educativo ya que los profesores suelen dar plena confianza a los manuales. Los libros de texto son poco partidarios de innovaciones ya que así las editoriales se aseguran la aprobación del profesorado que los solicita. Los profesores, por su parte, juegan un papel importante en el problema que estamos tratando. Principalmente porque en gran parte permanecen anclados en lo tradicional. Y los estudiantes muestran su desapego hacia la química señalando en especial los puntos siguientes (Solbes, Montserrat y Furió, 2007):

- Estudio muy teórico. Se deben aprender las reacciones químicas en el libro y nunca en el laboratorio.

- Dificultad. Como consecuencia, los alumnos optan a menudo por asignaturas que creen más sencillas y atractivas.

- Desconexión con la realidad. Los alumnos no encuentran que la química afecte a ningún aspecto de su vida.

- Falta de salidas profesionales.

Aún así, se sigue exigiendo a los alumnos que piensen, razonen y trabajen como científicos sin haberles proporcionado las estrategias adecuadas para actuar de esa manera (Chamizo, Nieto y Sosa, 2004).

Los objetivos de la enseñanza de la química son muy diversos. Hernández y Palacín (1993) consideran como objetivos generales básicos de nuestra asignatura los siguientes:

- Contribuir a formar individuos con capacidad de pensar por sí mismos. 
- Ayudar a potenciar al máximo las capacidades del alumno.

- Favorecer la familiarización con el método científico.

- Ayudar a la comprensión de la naturaleza a partir de la estructura de la materia y sus transformaciones.

- Conseguir en el alumno una visión crítica de la ciencia y de sus aplicaciones.

El profesor tiene un papel muy importante intentando enseñar contenidos de química y a la vez pretendiendo conseguir en el alumno motivación e interés hacia la asignatura. Aunque llevar a cabo todo esto no es tarea fácil. En primer lugar porque hay que tener en cuenta que el alumno no viene con la mente en blanco, sino con una serie de ideas previas o concepciones alternativas que deben tenerse en cuanta (Hierrezuelo y Montero, 1991). En química estas ideas erróneas han sido bien estudiadas por diversos autores (p.ej. Driver, 1985; Nakhleh, 1992). Por lo que respecta a motivación, los profesores utilizan métodos basados en su propia experiencia, lo cual no suele ser recomendable. Para ello deberían cambiar el método tradicional de enseñanza basado en gran parte en la memorización de la teoría, por otros más activos, aprovechables y amenos. El método más utilizado actualmente en ciencia es el aprendizaje por investigación (Montiano, 2010). El método se enmarca dentro del modelo constructivista, a cuya base se encuentra la teoría cognitiva del aprendizaje (Rodrigo y Cubero, 2000).

\section{La enseñanza de la formulación}

Al comenzar el estudio de la química, como de cualquier otra ciencia, es imprescindible el conocimiento de su lenguaje propio para conseguir comprender las exposiciones de cualquier manual (Latorre, 1999). Los investigadores han señalado tres niveles de representación en química: macroscópico, microscópico y simbólico (Gabel, 1998). Asimismo se ha constatado que muchos alumnos tienen grandes dificultades de comprensión de las representaciones simbólicas, entre las que se incluye la formulación (Johnstone, 1993). La causa apuntada es que los alumnos tienen a permanecer en niveles sensoriales y, por ello, tienen son incapaces de interpretar representaciones simbólicas como la formulación (Griffiths, 1992).

Es bien conocido que entre las diferentes áreas de ciencias existen contenidos que resultan tediosos y complejos de estudiar para una mayoría del alumnado (Muñoz, 2010). Esto es lo que ocurre con la formulación química. A falta de ella la química se construye sobre una base incompleta que puede impedir un aprendizaje adecuado. Por ello debe quedar bien asimilada, al menos sus principios básicos, desde el comienzo, sin esperar a aprenderla en cursos superiores. A este respecto debe señalarse que hay profesores para los que la enseñanza de la formulación no es considerada primordial, y prefieren impartir otros temas de contenido formativo que les parecen más adecuados. En el extremo opuesto, otros, por el contrario consideran la formulación como un contenido fundamental de la 
química, y obligan al alumno a aprender fórmulas de sustancias poco usuales y de algunas que ni existen (Hernández y Palacín, 1993).

Otro aspecto a tratar son los libros de texto. En ellos se presenta la formulación como un tipo de lenguaje, sin insistir en la interpretación y significado que encierra la fórmula de una sustancia. La mayoría de los ejercicios propuestos en los libros de texto, que los alumnos resuelven de manera mecánica, no contribuyen a enderezar esta situación (Taskin y Bernholt, 2012). Puede comprenderse, pues, el comentario habitual de los alumnos sobre esta cuestión "Me sé de memoria la fórmula química, pero no entiendo su significado" (De Jong, 1996, p. 279).

Para comenzar adecuadamente el aprendizaje de la formulación química, el alumno debe entender que la fórmula química de una sustancia o compuesto es una forma de expresar qué elementos forman parte de un compuesto y qué proporción guardan al combinarse. Pero lo que ocurre en la realidad, según la investigación didáctica, es que una buena parte de los alumnos sigue aún viendo las fórmulas como abreviaturas de nombres (Ben-Zvi, Eylon y Silberstein, 1988).

Por otra parte, aunque se han estudiado las dificultades que tienen los alumnos para entender el universo microscópico y el lenguaje mediante representaciones simbólicas de las sustancias, su puesta en práctica ha hecho caso omiso a las posibles soluciones del problema.

\subsection{MARCO CURRICULAR}

Puesto que el tema de nuestra investigación es la formulación química, hemos comenzado buscando todo lo que aparece al respecto en el Real Decreto 1631/2006 del 29 de diciembre, donde se establecen las enseñanzas mínimas correspondientes a la Educación Secundaria Obligatoria (BOE de 05-01-2007). Del citado Decreto se han revisado preámbulos, objetivos, competencias, contenidos y criterios de evaluación.

La etapa educativa en la que se inserta nuestro trabajo señala en el citado Decreto su finalidad respecto a la ciencia: "La educación secundaria obligatoria ha de facilitar a todas las personas una alfabetización científica que haga posible la familiarización con la naturaleza y las ideas básicas de la ciencia." (Ministerio, 2006, p. 690). Y más adelante aclara las connotaciones de educación científica para la ciudadanía: "La ciencia en esta etapa debe estar próxima al alumnado y favorecer su familiarización progresiva con la cultura científica, llevándole a enfrentarse a problemas abiertos y a participar en la construcción y puesta a prueba de soluciones tentativas fundamentadas. Ésta es la alfabetización científica que requiere la formación ciudadana, pero es también la mejor formación científica inicial que puede recibir un futuro científico, pues permite salir al paso de visiones 
deformadas y empobrecidas, puramente operativas de la ciencia, que generan un rechazo hacia la misma que es necesario superar." (Ministerio, 2006, p. 691).

Como objetivo de asignatura presente en la materia de Ciencias de la naturaleza, y relacionado con el tema de la investigación, aparece el siguiente: “Comprender y expresar mensajes con contendido científico utilizando un lenguaje oral y escrito con propiedad." (Ministerio, 2006, p. 693).

Las competencias básicas señaladas por el BOE y que pueden afectar más particularmente a nuestro tema son las siguientes:

"La mayor parte de los contenidos de Ciencias de la naturaleza tiene una incidencia directa en la competencia en el conocimiento y la interacción con el mundo físico. Precisamente el mejor conocimiento del mundo físico requiere el aprendizaje de los conceptos y procedimientos esenciales de cada una de las ciencias de la naturaleza y el manejo de las relaciones entre ellos: de causalidad o de influencia, cualitativas o cuantitativas, y requiere asimismo la habilidad para analizar sistemas complejos, en los que intervienen varios factores." (Ministerio, 2006, p. 691). El tema que se está analizando está comprendido en el área de ciencias, por lo que esta competencia es una de las principales.

"La competencia matemática está íntimamente asociada a los aprendizajes de las Ciencias de la naturaleza. La utilización del lenguaje matemático para cuantificar los fenómenos naturales, para analizar causas y consecuencias y para expresar datos e ideas sobre la naturaleza proporciona contextos numerosos y variados para poner en juego los contenidos asociados a esta competencia y, con ello, da sentido a esos aprendizajes." (Ministerio, 2006, p. 692). En formulación química están implícitas relaciones matemáticas, como más adelante veremos.

"El trabajo científico tiene también formas específicas para la búsqueda, recogida, selección, procesamiento y presentación de la información que se utiliza además en muy diferentes formas: verbal, numérica, simbólica o gráfica. La incorporación de contenidos relacionados con todo ello hace posible la contribución de estas materias al desarrollo de la competencia en el tratamiento de la información y competencia digital." (Ministerio, 2006, p. 692). La formulación química es en esencia una representación simbólica, basada en los símbolos químicos de los elementos. Hoy en día, el uso de las nuevas tecnologías de la información ayuda a dar respuestas y facilitar el aprendizaje de prácticamente cualquier área en la que se necesite ayuda. Por ello, la búsqueda de información acerca del tema podrá ayudar a mejorar el aprendizaje de la formulación. 
"La contribución de esta materia a la competencia en comunicación lingüística se realiza a través de (...) la adquisición de la terminología específica sobre los seres vivos, los objetos y los fenómenos naturales que hace posible comunicar adecuadamente una parte muy relevante de las experiencia humana y comprender suficientemente lo que otros expresan sobre ella." (Ministerio, 2006, p. 692). Esta es la competencia que más nos interesa, ya que la principal utilidad de la formulación y nomenclatura química es aprender y saber usar el lenguaje y la comunicación en este ámbito de la ciencia.

"Los contenidos asociados a la forma de construir y transmitir el conocimiento científico constituyen una oportunidad para el desarrollo de la competencia para aprender a aprender. En el aprendizaje a lo largo de la vida, en el caso del conocimiento de la naturaleza, se va produciendo por la incorporación de informaciones provenientes en unas ocasiones de la propia experiencia y en otras de medios escritos o audiovisuales." (Ministerio, 2006, p. 692). En el aprendizaje de la formulación química es efectivo, una vez que se adquieren los procesos básicos, que el alumno se autoejercite, complementando las tareas señaladas por el profesor. Esto puede llevarse a cabo sobre todo mediante la realización de ejercicios, donde el alumno puede comprobar sus errores y así mejorar su aprendizaje.

Como se puede observar, la formulación química no desarrolla todas las competencias básicas. Esto es debido a que siendo un tema muy específico, difícilmente puede abarcarlas todas.

Con respecto a los contenidos, vamos a reproducir los programas del tercer y cuarto curso de E.S.O. en la asignatura de Física y Química. Hemos señalado los títulos de los bloques y hemos recogido las secciones de estos que pueden resultarnos de interés.

El tercer curso consta de cuatro bloques (Ministerio, 2006, p. 696):

Bloque 1. Contenidos comunes.

Utilización de estrategias propias del trabajo científico.

Bloque 2. Diversidad y unidad de estructura de la materia.

La naturaleza corpuscular de la materia.

La teoría atómico-molecular de la materia.

Revisión de los conceptos de mezcla y sustancia.

Sustancias simples y compuestas.

Introducción del concepto de elemento químico. 
Bloque 3. Estructura interna de las sustancias.

Propiedades eléctricas de la materia.

Estructura del átomo. Modelos de Thomson y de Rutherford.

Bloque 4. Cambios químicos y sus repercusiones.

Reacciones químicas y su importancia.

Descripción del modelo atómico-molecular para explicar las reacciones químicas. Interpretación de la conservación de la masa. Representación simbólica.

Como vemos, todos los contenidos son de química. Entre ellos no figura de modo explícito la formulación y la nomenclatura.

El cuarto curso consta de cinco bloques. (Ministerio, 2006, p. 698-699). De ellos sólo el bloque 4 está centrado en la química. Los demás bloques están destinados a contenidos comunes y contenidos de física.

Bloque 1. Contenidos comunes.

Familiarización con las características básicas del trabajo científico

Bloque 2. Las fuerzas y los movimientos.

Bloque 3. Profundización en el estudio de los cambios.

Energía, trabajo y calor.

Las ondas.

Bloque 4. Estructura y propiedades de las sustancias.

La estructura del átomo. El sistema periódico de los elementos químicos.

El enlace químico: enlaces iónico, covalente y metálico.

Introducción a la formulación y nomenclatura de los compuestos binarios según las normas de la IUPAC.

Bloque 5. La contribución de la ciencia a un futuro sostenible

Vemos, pues, que es en el cuarto curso donde se establece de modo específico una introducción a la formulación pero sólo "de los compuestos binarios según las normas de la IUPAC".

Entre los criterios de evaluación de ambos cursos no hay ninguno sobre formulación. Únicamente hemos encontrado uno en 4 o curso referido a la tabla periódica. Lo hemos indicado ya que entre la formulación química y la tabla periódica existe una estrecha relación y nos servirá para evaluar la presentación de la formulación en los manuales. Es el siguiente: 
"Identificar las características de los elementos químicos más representativos de la tabla periódica, predecir su comportamiento químico al unirse con otros elementos, así como las propiedades de las sustancias simples y compuestas formadas.

Con este criterio se pretende comprobar que el alumnado es capaz de distribuir los electrones de los átomos en capas, justificando la estructura de la tabla periódica." (Ministerio, 2006, p. 700). 


\section{EL PROBLEMA DE INVESTIGACIÓN. OBJETIVOS}

\subsection{INTERROGANTES DE INVESTIGACIÓN}

Según lo expuesto en la Introducción (1.1), existe en la actualidad un problema muy extendido por prácticamente todos los países, que es la disminución drástica del número de estudiantes que eligen los estudios de ciencias. Las causas de este hecho apuntan a fallos importantes en la enseñanza de las ciencias, que provocan una ausencia de atracción de los alumnos hacia estos estudios, en general, y los de química muy en particular.

Nuestra investigación se sitúa en el seno de esta problemática. Prestamos atención a la enseñanza de la química en la actualidad y, más concretamente, a uno de los elementos sobre el que recae gran parte de la responsabilidad de este estado de cosas: la enseñanza de la formulación a nivel inicial.

Si tuviéramos que señalar un interrogante general de nuestra investigación, este sería:

¿Por qué la formulación química provoca rechazo en la mayor parte de los alumnos?

Este interrogante general podemos concretarlo en los siguientes:

* ¿Qué elementos (conceptuales y procedimentales) intervienen normalmente en la enseñanza de la formulación a nivel inicial?

* ¿Qué directrices proponen los documentos oficiales acerca de la formulación a nivel inicial ( $3^{\circ}-4^{\circ}$ de ESO)?

${ }^{*}$ ¿Cómo presentan los libros de texto la formulación a nivel inicial?

* ¿Qué planteamiento ofrecen los libros de texto para la enseñanza de la formulación a nivel inicial?

* ¿Cuáles son los elementos de su enseñanza que aparecen como poco procedentes? ¿Cómo podría mejorarse la situación? 


\subsection{OBJETIVOS}

Una vez planteado el problema y fijados los interrogantes de investigación, nos encontramos con la tarea de dar respuesta a dichos interrogantes. Para ello vamos ahora a fijar unos objetivos que van a guiar nuestra investigación a fin de dar cuenta de los interrogantes anteriores. Cada interrogante está relacionado con uno o varios objetivos.

Así pues, los objetivos que nos proponemos son los siguientes:

1. Señalar los contenidos conceptuales y procedimentales que normalmente intervienen en la enseñanza de la formulación y nomenclatura químicas a nivel inicial.

2. Estudiar en los documentos oficiales (BOE) las indicaciones propuestas acerca de la formulación.

3. Analizar exposiciones de formulación en libros de texto de $3^{\circ}$ y $4^{\circ}$ de ESO, mediante un protocolo que contenga los tópicos antes especificados.

4. Especificar cómo y qué contenidos de formulación presentan los manuales.

5. Especificar las tendencias de los manuales (que en la mayor parte de los casos serán las de los profesores) sobre el modo de enseñar la formulación.

6. En relación con el objetivo anterior, señalar algunos tópicos y estrategias claramente improcedentes de enseñanza de la formulación.

\subsection{DESARROLLO DEL TRABAJO}

1. Introducción.

Comenzamos con una exposición donde se traza un panorama general del estado de la cuestión acerca de la enseñanza de la Química, y más concretamente de la formulación (1.1). Dentro de este marco se especifican las directrices propuestas por el BOE (Decreto de Mínimos) para los cursos 3oㅡ y $4^{\circ}$ de ESO (1.2).

2. El problema de investigación. Objetivos.

En este tema se trazan los interrogantes de investigación (2.1) y, conforme a ellos, se señalan los objetivos que van a guiar nuestra investigación (2.2). Se presenta, además, el desarrollo en que va a exponerse el trabajo (2.3). 


\section{Fundamentos.}

Como paso previo a la investigación, es necesario estudiar los fundamentos en que se basa la enseñanza de la formulación, tanto conceptuales (3.1), como procedimentales (3.2), y los sistemas que suelen utilizarse para nombrar las sustancias (3.3).

\section{Metodología.}

Aquí nos introducimos de lleno en el proceso de investigación. Como vamos a investigar la manera en que aparece la formulación en los libros de texto, es imprescindible para ello fijar en primer lugar la muestra elegida (4.1). A continuación, estudiar los tópicos a considerar en el análisis (4.2), que serán los que han aparecido en el tema anterior, junto a otros más escolares (p.ej. actividades propuestas). Con ello se va a elaborar un protocolo de análisis (4.3) para ser aplicado a la muestra.

5. Resultados. Análisis de resultados.

De la aplicación del protocolo de análisis a la muestra se obtendrán unos resultados, que dan idea del tratamiento que proporcionan los manuales al tema de formulación (5.1). A continuación se procede al correspondiente estudio de estos resultados en cada uno de los tópicos considerados (5.2) y, como consecuencia, van a aparecer las orientaciones y tendencias de enseñanza de la formulación que ofrecen los manuales, de las cuales se señalarán algunas por improcedentes (5.3).

6. Conclusiones.

El trabajo se completa con unas conclusiones finales que van a recoger las aportaciones más esenciales de la investigación emprendida.

Bibliografía y Anexos.

La exposición se cierra con la correspondiente bibliografía utilizada, donde figuran también los manuales de la muestra. Igualmente se han incluido en los anexos algún fragmento característico de manuales de la muestra.

Incluimos en la tabla siguiente (Tabla 2.1) un resumen de las relaciones, ya comentadas, entre los apartados del presente tema. 


\begin{tabular}{|c|c|c|}
\hline $\begin{array}{c}\text { Interrogante de } \\
\text { investigación }\end{array}$ & Objetivo/s & $\begin{array}{c}\text { Desarrollo } \\
\text { (apartados) }\end{array}$ \\
\hline $\begin{array}{c}\text { Qué elementos } \\
\text { intervienen en la } \\
\text { enseñanza de la } \\
\text { formulación...? }\end{array}$ & 1 & $3.1 / 3.2 / 3.3$ \\
\hline $\begin{array}{c}\text { Qué directrices proponen } \\
\text { los documentos } \\
\text { oficiales...? }\end{array}$ & 2 & 1.2 \\
\hline $\begin{array}{c}\text { Cómo presentan los } \\
\text { contenidos los libros de } \\
\text { texto...? }\end{array}$ & $3 / 4$ & $4.1 / 4.2 / 4.3$ \\
$/ 5.1 / 5.2$
\end{tabular}

Tabla 2.1. Relación interrogantes - objetivos - desarrollo 


\section{FUNDAMENTOS}

\subsection{NOMENCLATURA Y FORMULACIÓN. CONCEPTOS DE BASE}

El tema de formulación y nomenclatura, objeto de nuestra investigación, es un tema complejo que descansa en una serie de conceptos básicos de fundamento químico. Debemos, pues, señalar estos conceptos y términos y considerarlos con cierto detalle.

En principio vamos a comenzar por el concepto de elemento. Las sustancias están formadas por combinaciones de otros cuerpos más básicos, que son los elementos. Los elementos no se pueden dividir en cuerpos más sencillos a través de procedimientos químicos. Un elemento está formado por átomos iguales, o más exactamente, por átomos de un valor de $\mathrm{Z}$ determinado. Cada elemento viene designado mediante por un símbolo químico, que tiene validez internacional. Consiste en una o dos letras (la primera con mayúscula) del nombre del elemento, algunas veces tomado del latín o el griego.

Los elementos están ordenados en la tabla periódica, de tal manera que los grupos o columnas poseen propiedades químicas similares. En la tabla periódica podemos ver la totalidad de los elementos y sus símbolos. Así, conociendo los símbolos de los elementos se podrán expresar las fórmulas de las sustancias en cuya composición intervienen, y construir el nombre de cada una siguiendo las normas de la nomenclatura química.

Debe tenerse en cuenta que existen dos tipos de elementos: los metales y los no metales. Los primeros muestran modos de combinación y propiedades diferentes a los segundos. A efectos de definir los compuestos y establecer sus fórmulas es necesario conocer si un elemento pertenece al grupo de los metales o de los no metales. Los primeros tienen tendencia a desprenderse de electrones y los segundos a captarlos.

Las similitudes y diferencias encontradas en las estructuras electrónicas de los elementos es la causa por la que encontramos propiedades periódicas a lo largo de la tabla periódica. Hoy en día, sabemos que la reactividad de un elemento depende de su estructura electrónica externa, debido a que en la formación de enlaces sólo intervienen los electrones más exteriores. Así, los elementos de un mismo grupo se caracterizan por poseer idéntico número de electrones en orbitales externos del mismo tipo $(s, p, d$ o $f)$, por lo que poseen una configuración electrónica similar.

Los elementos componentes de las sustancias se encuentran siempre en unas proporciones ponderales determinadas. Esto se debe a que, en un mismo 
compuesto, los átomos de dichos elementos se encuentran unidos en una misma proporción numérica.

Es conveniente marcar la diferencia entre dos términos fundamentales que en muchas ocasiones parecen confundirse: formulación y nomenclatura.

La formulación consiste en representar una sustancia de una manera simbólica. Ello se realiza utilizando los símbolos de los elementos que lo constituyen. La fórmula química nos indica cuáles son los elementos que forman la sustancia, y la cantidad o proporción de átomos en el compuesto. El objetivo de la formulación es asignar a una sustancia su fórmula química.

La nomenclatura consiste en nombrar una sustancia a partir de su fórmula química. Existe una serie de reglas utilizadas para asignar un nombre químico a las sustancias, según el tipo de combinación que se trate, que permiten asignar un nombre a cada sustancia para que quede definida de forma inequívoca. Veremos más adelante que estas normas son propias de tres sistemas de nomenclatura diferentes.

Es importante también tener claro el significado de la fórmula química. De una fórmula se puede deducir los elementos constituyentes de la sustancia, así como la proporción de átomos de cada tipo que intervienen y la relación ponderal de la combinación.

Por último, la clasificación de las sustancias por tipos se puede llevar a cabo de dos maneras: siguiendo un criterio analítico, o mediante el tipo de compuesto que forman. El criterio analítico clasifica a las sustancias en función del número de átomos que contenga la fórmula. Así surgiría la siguiente clasificación:

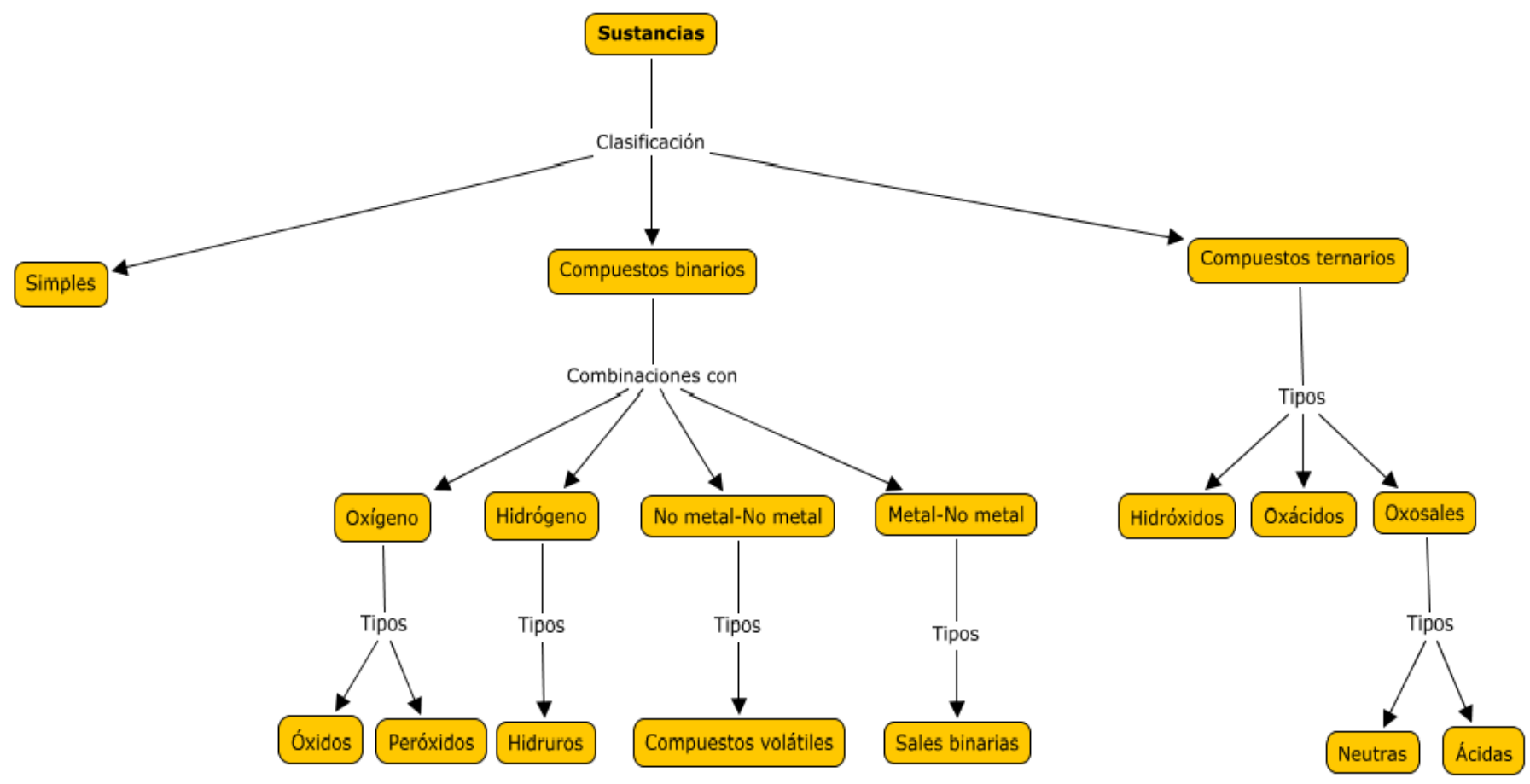


En cambio, si predomina el criterio del tipo de compuestos, la clasificación tendría lugar dependiendo de la función química que posea la molécula:

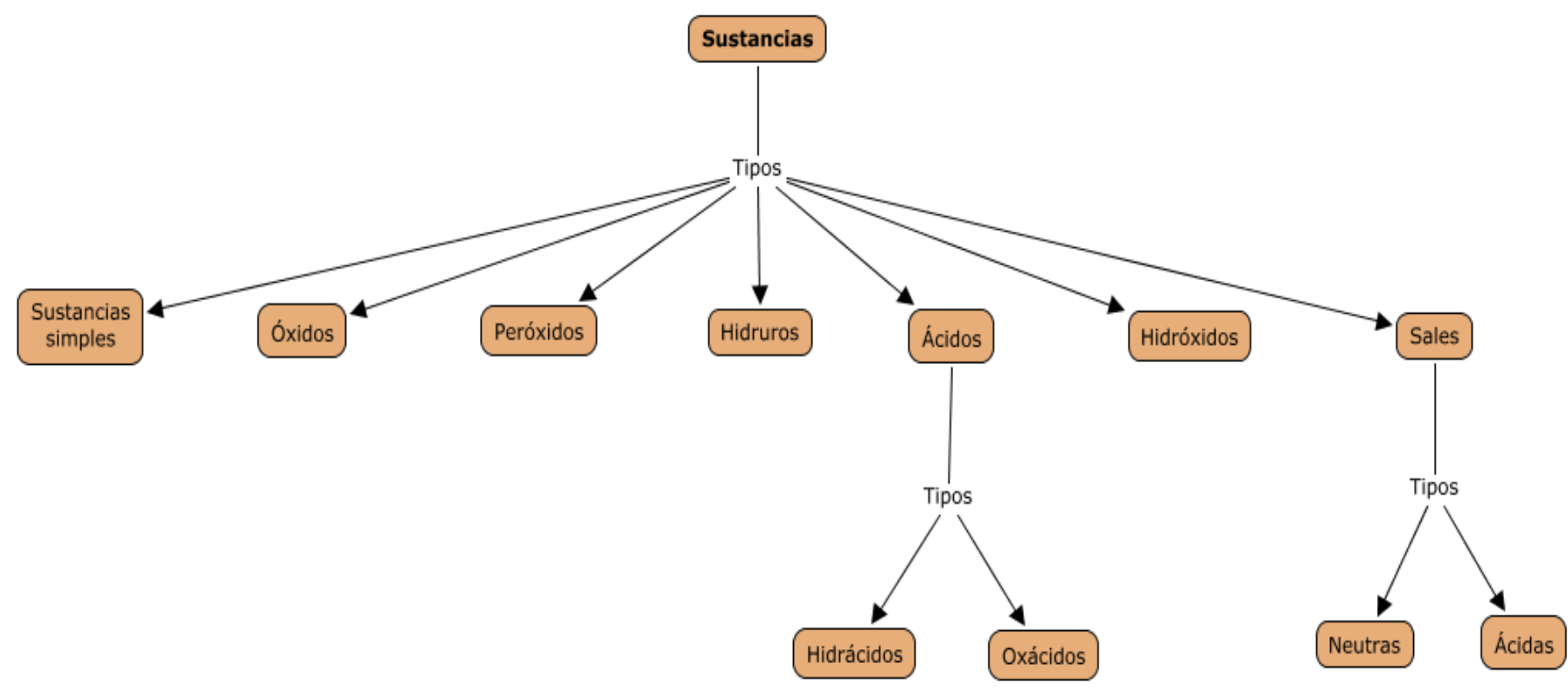

- Óxidos: Son compuestos binarios formados por oxígeno y cualquier elemento de la tabla periódica.

- Peróxidos: Son compuestos binarios que contienen un enlace oxígeno-oxígeno unido a otro elemento.

- Hidruros metálicos: Son compuestos binarios formados por metales e hidrógeno.

- Hidruros no metálicos o hidrácidos: Son compuestos binarios formados por un no metal e hidrógeno. Si el hidruro se encuentra en disolución acuosa se le denomina hidrácido.

- Compuestos no metal-no metal: Son combinaciones binarias de dos no metales distintos del oxígeno y el hidrógeno.

- Hidróxidos: Son compuestos formados por un metal y uno o varios grupos hidróxido $(\mathrm{OH})$.

- Ácidos oxácidos: Son compuestos formados por oxígeno, hidrógeno y un no metal (o un metal de transición).

- Sales binarias: Compuestos formados por un metal y un no metal. 
- Oxosales: Son combinaciones ternarias que pueden considerarse derivadas de los ácidos oxácidos, sustituyendo sus hidrógenos por un metal.

- Sales ácidas: Son combinaciones que pueden considerarse derivadas de sustituir por un metal parte de los hidrógenos presentes en un ácido.

\subsection{PROCEDIMIENTOS DE FORMULACIÓN}

Para obtener la fórmula química de cualquier sustancia existe un conjunto de normas útiles desarrolladas en los manuales. Antes de describir estos métodos vamos a introducir algunos conceptos de base que se deben tener en cuenta antes de comenzar a formular:

- El primer concepto importante es el concepto de valencia. La valencia se define como la capacidad de combinación que tiene el átomo de un elemento. Es un número natural que mide el número de enlaces que puede formar con otros átomos o grupos de átomos. Es útil conocer la valencia de cada átomo para posteriormente expresar cómo se combinan los átomos en la fórmula química.

- Otro concepto básico es el número de oxidación, el cual no se debe confundir con el concepto de valencia. El número de oxidación es un número entero y se define como la carga que tendría el átomo de un elemento si todos los enlaces de la molécula en la que interviene se hicieran iónicos. Como para ello se supone que el elemento más electronegativo se queda con los electrones del enlace, vemos que el concepto de número de oxidación está montado sobre el concepto de electronegatividad.

Existen unas reglas para calcular el número de oxidación:

$\checkmark$ El número de oxidación de un elemento libre ( $\mathrm{Zn}, \mathrm{H}_{2}, \mathrm{~N}_{2}$, etc.) es cero.

$\checkmark$ El número de oxidación de un ion monoatómico es igual a su carga.

$\checkmark$ El número de oxidación del hidrógeno es +1 , excepto en los hidruros metálicos que es -1 .

$\checkmark$ El número de oxidación del oxígeno es -2, excepto en los peróxidos que es -1 . 
$\checkmark$ El número de oxidación de los metales en los compuestos es igual a su valencia iónica: alcalinos, +1 ; alcalinotérreos, +2 , etc.

$\checkmark$ La suma algebraica de los números de oxidación de todos los átomos de un compuesto es igual a cero. Si se trata de un ion, esta suma debe ser igual a la carga del ion.

A menudo los términos de valencia y de número de oxidación son confundidos. Un error muy común es emplear el término de valencia para hacer referencia al número de oxidación, otorgándoles valores positivos y negativos a las valencias.

Una vez aclarados estos conceptos podemos describir los diversos métodos propuestos para deducir la fórmula química (García y Teijón, 1993; Josa, 1995; Peterson, 1987):

1. El primer procedimiento consiste en escribir en el caso de los compuestos binarios el símbolo químico de cada elemento o grupo, poniendo a la izquierda el elemento más electropositivo y a la derecha el más electronegativo. Seguidamente se colocan como subíndices las valencias o números de oxidación de cada elemento intercambiados de un elemento a otro. Por último, si estos subíndices poseen un denominador común se dividen por éste hasta que sean lo más reducidos posible.

En el caso de compuestos no binarios, se sigue el mismo mecanismo con los átomos y grupos que pudieran intervenir.

Ejemplos:

$$
\begin{gathered}
\text { Hidrógeno }(\mathrm{H}), \text { valencia }=1 \\
\text { Cloro }(\mathrm{Cl}), \text { valencia }=1 \\
\mathrm{H}_{1} \mathrm{Cl}_{1} \rightarrow \mathrm{HCl} \\
\text { Plomo }(\mathrm{Pb}), \text { valencia }=4 \\
\text { Oxígeno }(\mathrm{O}), \text { valencia }=2 \\
\mathrm{~Pb}_{2} \mathrm{O}_{4} \rightarrow \mathrm{PbO}_{2} \\
\text { Sodio }(\mathrm{Na}), \mathrm{valencia}^{2} 1 \\
\text { Anión carbonato }\left(\mathrm{CO}_{3}^{2-}\right), \text { valencia }=2 \\
\mathrm{Na}_{2} \mathrm{CO}_{3}
\end{gathered}
$$

2. Otro procedimiento se basa en aprender la fórmula general para cada tipo de compuesto y simplificar los subíndices como en el método anterior cuando sea posible. Las fórmulas generales a tener en cuenta pueden ser:

$$
\text { Óxidos } \rightarrow \mathrm{X}_{2} \mathrm{O}_{\mathrm{n}}
$$




$$
\begin{aligned}
& \text { Hidruros } \rightarrow \mathrm{XH}_{\mathrm{n}} \\
& \text { Hidrácidos } \rightarrow \mathrm{H}_{\mathrm{n}} \mathrm{X} \\
& \text { Sales binarias } \rightarrow \mathrm{M}_{\mathrm{n}} \mathrm{N}_{\mathrm{m}} \\
& \text { Hidróxidos } \rightarrow \mathrm{M}(\mathrm{OH})_{\mathrm{m}} \\
& \text { No metal-no metal } \rightarrow \mathrm{X}_{\mathrm{y}} \mathrm{Y}_{\mathrm{x}} \\
& \text { Oxosales } \rightarrow \mathrm{Y}_{\mathrm{a}}\left(\mathrm{X}_{\mathrm{b}} \mathrm{O}_{\mathrm{c}}\right)_{\mathrm{y}}
\end{aligned}
$$

Ejemplos:

$$
\begin{gathered}
\text { Hidróxido de calcio: } \\
\text { Valencia del calcio }(\mathrm{Ca})=2, \text { por tanto } m=2 \\
M(\mathrm{OH})_{m} \rightarrow \mathrm{Ca}(\mathrm{OH})_{2} \\
\text { Hidruro de sodio } \\
\text { Valencia del sodio }(\mathrm{Na})=1, \text { por tanto } n=1 \\
\mathrm{XH}_{n} \rightarrow \mathrm{NaH}_{1} \rightarrow \mathrm{NaH} \\
\text { Sulfato de aluminio } \\
\text { Deriva del ácido sulfúrico: } \mathrm{H}_{2} \mathrm{SO}_{4}, \text { por tanto } b=1, c=4, a=2 \\
\text { Valencia del aluminio }\left(\mathrm{Al}^{\mathrm{a}}=3, \text { por tanto } y=3\right. \\
\mathrm{Y}_{\mathrm{a}}\left(\mathrm{X}_{\mathrm{b}} \mathrm{O}_{\mathrm{c}}\right)_{\mathrm{y}} \rightarrow \mathrm{Al}_{2}\left(\mathrm{SO}_{4}\right)_{3}
\end{gathered}
$$

La fórmula general de los oxácidos sería la siguiente:

$$
\text { Oxácidos } \rightarrow \mathrm{H}_{\mathrm{a}} \mathrm{X}_{\mathrm{b}} \mathrm{O}_{\mathrm{c}}
$$

Pero los oxácidos no se pueden formular de esta manera tan sencilla como ocurre con el resto de los compuestos. El procedimiento para ellos lo describiremos después.

3. El tercer método estaría basado en el número de oxidación. Consistiría en escribir la fórmula química teniendo en cuenta que la suma de los números de oxidación de cada átomo de la molécula debe ser igual a cero.

Ejemplos:

Óxido de calcio

Oxígeno (O), número de oxidación $=-2$

Calcio (Ca), número de oxidación $=+2$

La fórmula ha de ser $\mathrm{CaO}$

porque: $+2-2=0$

Fórmula de un oxácido cuyo átomo central sea cloro con número de oxidación +3 Hidrógeno $(H)$, número de oxidación $=+1$ 


$$
\begin{gathered}
\text { Cloro (Cl), número de oxidación }=+3 \\
\text { Oxígeno (O), número de oxidación }=-2 \\
\text { La fórmula ha de ser } \mathrm{HClO}_{2} \\
\text { porque: } 1+3+(-2) \cdot 2=0
\end{gathered}
$$

(Anexo I)

\section{Formulación de los oxácidos.}

En el caso concreto de los oxácidos hay varios procedimientos que suelen darse para llegar a su fórmula:

$\checkmark$ El primer método consiste en partir del óxido correspondiente y sumarle una o varias moléculas de agua. Si es necesario se simplifican los subíndices.

$$
\begin{gathered}
\mathrm{SO}_{3}+\mathrm{H}_{2} \mathrm{O} \rightarrow \mathrm{H}_{2} \mathrm{SO}_{4} \\
\mathrm{P}_{2} \mathrm{O}_{5}+3 \mathrm{H}_{2} \mathrm{O} \rightarrow \mathrm{H}_{6} \mathrm{P}_{2} \mathrm{O}_{8} \rightarrow \mathrm{H}_{3} \mathrm{PO}_{4}
\end{gathered}
$$

$\checkmark$ Otro de ellos consiste en memorizar tablas como la siguiente, que recopilan las fórmulas de los ácidos más comunes en función de la valencia del átomo central:

\begin{tabular}{|c|c|c|}
\hline Valencia & Fórmula & Ejemplos \\
\hline I & $\mathrm{HXO}$ & $\mathrm{HClO}$ \\
II & $\mathrm{H}_{2} \mathrm{XO}_{2}$ & $\mathrm{H}_{2} \mathrm{SO}_{2}$ \\
III & $\mathrm{HXO}_{2}$ & $\mathrm{HClO}_{2}$ \\
IV & $\mathrm{H}_{2} \mathrm{XO}_{3}$ & $\mathrm{H}_{2} \mathrm{SO}_{3}$ \\
V & $\mathrm{HXO}_{3}$ & $\mathrm{HClO}_{3}$ \\
VI & $\mathrm{H}_{2} \mathrm{XO}_{4}$ & $\mathrm{H}_{2} \mathrm{SO}_{4}$ \\
VII & $\mathrm{HXO}_{4}$ & $\mathrm{HClO}_{4}$ \\
\hline
\end{tabular}

Tabla 3.1. Fórmula general de oxácidos en función de la valencia

Hay otros métodos, muy minoritarios, y alguno de ellos muy poco recomendable. El que se da a continuación propone el siguiente procedimiento:

1. Se escriben los símbolos de los elementos en el orden correcto: HXO

2. Para averiguar el número de átomos de oxígeno, se toma el número de oxidación del elemento central, se divide entre dos, se desprecia la parte decimal y se le suma 1 . (¡Atención a esto!)

3. El número de átomos de hidrógeno se determina teniendo en cuenta que la suma de los números de oxidación ha de ser 0 :

$$
\text { n⿳o de } \mathrm{H}=2 \times \text { no de } \mathrm{O}-\mathrm{n}^{\mathrm{o}} \text { ox de } \mathrm{X} \text { (¡La fórmula mágica!) }
$$


Ejemplo, ácido clórico, donde 끄ox $(\mathrm{Cl})=+5$ :
1. $\mathrm{HClO}$
2. $5 / 2=2,5$ $\mathrm{n} \stackrel{\mathrm{o}}{(0)}=2+1=3$
3. $\mathrm{n}-\mathrm{o}(\mathrm{H})=2 \times 3-5=1$

Por tanto: $\mathrm{HClO}_{3}$

\subsection{SISTEMAS DE NOMENCLATURA}

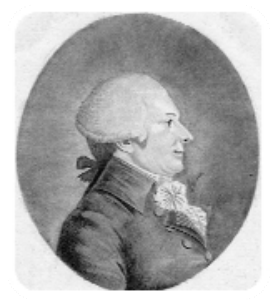

"Es necesario un método constante de denominación que ayude a la inteligencia y alivie la memoria" (Guyton de Morveau).

Existen muchas sustancias químicas y cada día surgen muchísimas más en los laboratorios. Cada sustancia, molécula o compuesto es único y debe tener un nombre propio correspondiente. Para facilitar su identificación se han creado unas normas para nombrar a todos los compuestos químicos bajo las mismas reglas. La nomenclatura puede definirse como la terminología empleada para hacer referencia a las sustancias y compuestos químicos. La IUPAC es el organismo internacional encargado de aprobar los nombres de las diferentes sustancias químicas y de fijar y recomendar las normas generales de nomenclatura química.

Los métodos que vamos a definir reúnen y nombran a los compuestos inorgánicos, ya que la nomenclatura de los compuestos orgánicos sigue otra estructura. Los compuestos químicos inorgánicos se nombran atendiendo a la función química que contengan y al número de elementos químicos que contengan. Actualmente se admiten tres sistemas diferentes de nomenclatura (García y Teijón, 1993; Josa, 1995; Peterson, 1987), los cuales son:

- Nomenclatura sistemática

- Nomenclatura de Stock

- Nomenclatura tradicional

La nomenclatura sistemática es la recomendada por la IUPAC (Latorre, 1999). También es conocida como nomenclatura estequiométrica. Nombra los compuestos químicos utilizando prefijos numéricos griegos que muestran el número de átomos de un mismo elemento presentes en la fórmula.

a) Óxidos: Se utiliza la palabra "óxido" en primer lugar. La proporción atómica se indica con los prefijos numerales. Seguidamente, tras la palabra "de" se nombra el otro elemento utilizando los prefijos multiplicativos que indican el número de átomos. 
Ejemplos:

$\mathrm{FeO} \rightarrow$ Monóxido de hierro

$\mathrm{Fe}_{3} \mathrm{O}_{4} \rightarrow$ Tetraóxido de trihierro

b) Hidruros metálicos: Se utiliza la palabra "hidruro" en primer lugar. La proporción atómica se indica con los prefijos numerales. Todo idéntico al caso anterior.

Ejemplos:

$\mathrm{NiH}_{3} \rightarrow$ Trihidruro de níquel

$\mathrm{BaH}_{2} \rightarrow$ Dihidruro de bario

c) Compuestos no metal - no metal: Utiliza el sufijo "-uro" para el elemento más electronegativo. Seguidamente, tras la palabra "de", se nombra el elemento menos electronegativo. Por lo demás, todo idéntico al caso anterior.

Ejemplos:

$\mathrm{As}_{2} \mathrm{Se}_{3} \rightarrow$ Triseleniuro de diarsénico

$\mathrm{CS}_{2} \rightarrow$ Disulfuro de carbono

Las combinaciones binarias de no metales con hidrógeno son los "hidruros no metálicos" o "hidrácidos": Se comienza nombrando el elemento distinto de hidrógeno con el sufijo "-uro" seguido "de hidrógeno".

Ejemplos:

$H F \rightarrow$ Fluoruro de hidrógeno

$\mathrm{HCl} \rightarrow$ Cloruro de hidrógeno

d) Sales binarias: Se nombran de la misma forma que los compuestos no metal - no metal.

Ejemplos:

$\mathrm{FeCl}_{3} \rightarrow$ Tricloruro de hierro

$\mathrm{Li}_{2} \mathrm{~S} \rightarrow$ Monosulfuro de dilitio o sulfuro de dilitio

e) Hidróxidos: Se utiliza la palabra "hidróxido" en primer lugar. La proporción atómica se indica con los prefijos numerales. Seguidamente, tras la palabra "de" se nombra el otro elemento utilizando los prefijos multiplicativos que indican el número de átomos. 


\section{Ejemplos:}

$\mathrm{Ca}(\mathrm{OH})_{2} \rightarrow$ Dihidróxido de calcio

$\mathrm{NaOH} \rightarrow$ Monohidróxido de sodio o hidróxido de sodio

f) Ácidos oxácidos: Se indica el número de átomos de oxígeno con el prefijo correspondiente, seguido de la partícula "oxo", unida al nombre del no metal o elemento central y el sufijo "-ato" y los correspondientes prefijos numerales, a continuación se agrega la palabra "de" y por último se añade el hidrógeno con los prefijos multiplicativos correspondientes.

Ejemplos:

\section{$\mathrm{H}_{2} \mathrm{SO}_{4} \rightarrow$ Tetraoxosulfato de dihidrógeno \\ $\mathrm{H}_{2} \mathrm{CO}_{3} \rightarrow$ Trioxocarbonato de dihidrógeno}

g) Oxosales: Se escribe el nombre del anión sin la carga, si es necesario con los prefijos "bis-", "tris-", "tetrakis-", "pentakis-", "hexakis-", etc. que nos indican la repetición del anión poliatómico. Seguido del catión, con los prefijos que nos indican la repetición del catión.

\section{Ejemplos:}

$\mathrm{NaNO}_{2} \rightarrow$ Dioxonitrato de sodio

$\mathrm{Ca}\left(\mathrm{ClO}_{2}\right)_{2} \rightarrow$ Bis[dioxoclorato] de calcio

h) Sales ácidas: Se comienza con la palabra "hidrógeno". A continuación y entre paréntesis se nombra el anión (sin indicar la carga) y, tras la palabra "de" se nombra el catión. La proporción de ambos constituyentes se indica mediante los prefijos multiplicativos. Cuando el nombre de un constituyente comienza por un prefijo multiplicativo se usan los prefijos de cantidad alternativos ("bis-", "tris-", "tetrakis-”, "pentakis-", etc.), Además el nombre se coloca entre corchetes al utilizar los prefijos alternativos de cantidad.

Ejemplos:

$\mathrm{CuHSO}_{4} \rightarrow$ Hidrogeno(tetraoxosulfato) de cobre

$\mathrm{Mg}\left(\mathrm{H}_{2} \mathrm{PO}_{4}\right)_{2} \rightarrow$ Bis[dihidrogeno(tetraoxofosfato)] de magnesio

La nomenclatura de Stock se distingue en que escribe al final del nombre de los compuestos, la valencia del elemento con números romanos entre paréntesis, en el caso de que el elemento posea más de una. 
a) Óxidos: Se utiliza la palabra "óxido" en primer lugar y se le añade el nombre del elemento restante colocando entre paréntesis y con números romanos la valencia.

Ejemplos:

$\mathrm{Cu}_{2} \mathrm{O} \rightarrow$ Óxido de cobre (I)

$\mathrm{CO}_{2} \rightarrow$ Óxido de carbono (IV)

b) Hidruros metálicos: Se utiliza la palabra "hidruro" en primer lugar y se le añade el nombre del elemento restante colocando entre paréntesis y con números romanos la valencia.

Ejemplos:

$\mathrm{PbH}_{4} \rightarrow$ Hidruro de plomo (IV)

$\mathrm{BeH}_{2} \rightarrow$ Hidruro de berilio

c) Compuestos no metal - no metal: Para nombrar se utiliza el elemento más electronegativo con el sufijo "-uro" y se le añade el nombre del más electropositivo colocando entre paréntesis su valencia.

Ejemplos:

$\mathrm{PCl}_{3} \rightarrow$ Cloruro de fósforo (III)

$\mathrm{BrF}_{5} \rightarrow$ Fluoruro de bromo (V)

Los hidruros no metálicos o hidrácidos se nombran igual que en la nomenclatura sistemática.

Ejemplos:

$\mathrm{H}_{2} \mathrm{Se} \rightarrow$ Seleniuro de hidrógeno

$\mathrm{H}_{2} \mathrm{Te} \rightarrow$ Telururo de hidrógeno

d) Sales binarias: Se nombran de la misma forma que los compuestos no metal - no metal.

Ejemplos:

$\mathrm{AuI}_{3} \rightarrow$ Yoduro de oro (III)

$\mathrm{MgCl}_{2} \rightarrow$ Cloruro de magnesio

e) Hidróxidos: Se utiliza la palabra "hidróxido" en primer lugar y se le añade el nombre del elemento restante colocando entre paréntesis y con números romanos su valencia. 
Ejemplos:

$\mathrm{Ge}(\mathrm{OH})_{2} \rightarrow$ Hidróxido de germanio (II)

$\mathrm{Sn}(\mathrm{OH})_{4} \rightarrow$ Hidróxido de estaño (IV)

f) Ácidos oxácidos: Se nombra al no metal con el sufijo “-ato", luego el número de valencia del no metal y por último se agrega "de hidrógeno".

Ejemplos:

$\mathrm{HClO}_{4} \rightarrow$ Clorato (VII) de hidrógeno

$\mathrm{H}_{2} \mathrm{SO}_{2} \rightarrow$ Sulfato (II) de hidrógeno

Otra forma de nombrarlos con la nomenclatura de Stock comenzaría con la palabra "ácido" y se escriben los subíndices del oxígeno (oxo) y del no metal y se terminan en -ico, poniendo el $\mathrm{n}$ o de oxidación del no metal entre paréntesis y con números romanos.

Ejemplos:

$\mathrm{H}_{3} \mathrm{PO}_{4}$ : ácido tetraoxofosfórico $(\mathrm{V})$

$\mathrm{H}_{2} \mathrm{Cr}_{2} \mathrm{O}_{7}$ : ácido heptaoxodicrómico (VI)

g) Oxosales: Se utiliza como nombre genérico el nombre del no metal o elemento central con el sufijo "-ato". Después del nombre general se indica la valencia del no metal con números romanos, y luego como nombre especifico se utiliza el nombre del metal.

\section{Ejemplos:}

$\mathrm{Fe}\left(\mathrm{ClO}_{3}\right)_{3} \rightarrow$ Clorato ( $\mathrm{V}$ ) de hierro (III)

$\mathrm{Mg}(\mathrm{BrO})_{2} \rightarrow$ Bromato (I) de magnesio

h) Sales ácidas: Se antepone la palabra "hidrógeno" al nombre de la sal, indicando con prefijos numerales el número de átomos de hidrógeno que quedan sin sustituir.

Ejemplos:

$\mathrm{Fe}\left(\mathrm{H}_{2} \mathrm{PO}_{4}\right)_{3} \rightarrow$ Dihidrogenofosfato (V) de hierro (III)

$\mathrm{NaHSO}_{4} \rightarrow$ Hidrogenosulfato (VI) de sodio

La nomenclatura tradicional insiste en los tipos de compuestos y utiliza una serie de prefijos y sufijos propios. 
o Si el metal presente en un compuesto (a veces el no metal en compuestos binarios) posee una única valencia, en el nombre del compuesto aparece el de este elemento precedido de la palabra "de". También se puede utilizar el sufijo "-ico".

o Si tiene dos valencias:

o Mayor: "-ico"

o Menor: "-oso"

o Tres valencias:

o Mayor: "-ico"

o Intermedia: "-oso"

o Menor: "Hipo-...-oso"

o Cuatro valencias:

o Mayor: "Per-...-ico"

o Media alta: "-ico"

o Media baja: "-oso"

o Menor: "Hipo-...-oso"

a) Óxidos: Se nombran comenzando por la palabra "óxido" continuando con el nombre del metal añadiendo los prefijos y sufijos anteriores atendiendo a las valencias de este elemento. Si el elemento es un no metal, entonces sustituiremos la palabra "óxido" por la palabra "anhídrido".

Ejemplos:

$\mathrm{TeO}_{2} \rightarrow$ Anhídrido teluroso

$\mathrm{CaO} \rightarrow$ Óxido cálcico

b) Hidruros metálicos: Se nombran comenzando por la palabra "hidruro" continuando con el nombre del metal añadiendo los prefijos y sufijos anteriores atendiendo a las valencias de este elemento.

Ejemplos:

KH $\rightarrow$ Hidruro potásico o hidruro de potasio

$\mathrm{FeH}_{2} \rightarrow$ Hidruro ferroso

c) Compuestos no metal - no metal: Para nombrar se utiliza el elemento más electronegativo con el sufijo "-uro" y se le añade el nombre del más electropositivo utilizando los prefijos y sufijos indicados anteriormente.

Ejemplos:

$B_{2} S_{3} \rightarrow$ Sulfuro bórico 
- Para los hidruros no metálicos o hidrácidos, la nomenclatura tradicional los nombra en disolución acuosa, comenzando por la palabra "ácido" seguida del nombre del elemento distinto de hidrógeno añadiéndole el sufijo “hídrico".

Ejemplos:

$\mathrm{HBr} \rightarrow$ Ácido bromhídrico

$\mathrm{H}_{2} \mathrm{~S} \rightarrow$ Ácido sulfhídrico

d) Sales binarias: Se nombran de la misma manera que los compuestos no metal-no metal.

Ejemplos:

$\mathrm{NaCl} \rightarrow$ Cloruro sódico o cloruro de sodio

$\mathrm{K}_{2} \mathrm{~S} \rightarrow$ Sulfuro potásico o sulfuro de potasio

e) Hidróxidos: Se nombran comenzando por la palabra "hidróxido" continuando con el nombre del metal añadiendo los prefijos y sufijos anteriores atendiendo a las valencias de este elemento.

Ejemplos:

$\mathrm{CuOH} \rightarrow$ Hidróxido cuproso

LiOH $\rightarrow$ Hidróxido lítico o hidróxido de litio

f) Ácidos oxácidos: Hay que conocer todos los estados de oxidación que puede tener el elemento central. Para nombrarlos, se antepone la palabra "ácido" a la raíz del nombre del elemento con los prefijos y sufijos correspondientes.

Ejemplos:

$\mathrm{HClO}_{2} \rightarrow$ Ácido cloroso

$\mathrm{HBrO}_{3} \rightarrow$ Ácido brómico

g) Oxosales: En el caso de las oxosales, si el ácido de origen termina en "-ico" la sal se nombra con "-ato" y si termina en "-oso" la sal se nombra con "-ito", continuando con el nombre del metal con los prefijos y sufijos correspondientes a esta nomenclatura.

Ejemplos:

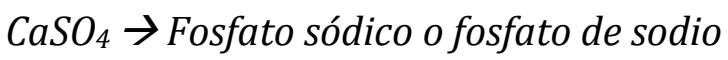


$\mathrm{NaClO}_{4} \rightarrow$ Perclorato sódico o perclorato de sodio

$\mathrm{Fe}\left(\mathrm{ClO}_{3}\right)_{2} \rightarrow$ Clorato ferroso

h) Sales ácidas: Se nombran como las sales neutras intercalando la expresión "ácido de" o también con el prefijo "bi-", y a continuación el metal con los prefijos y sufijos propios de esta nomenclatura según sea su valencia.

Ejemplos:

$\mathrm{NaHCO}_{3} \rightarrow$ Bicarbonato sódico o carbonato ácido de sodio

$\mathrm{FeHPO}_{4} \rightarrow$ Fosfato ácido ferroso

$\mathrm{Ca}(\mathrm{HS})_{2} \rightarrow$ Sulfuro ácido de calcio

Tanto en la nomenclatura de Stock como en la nomenclatura tradicional, a partir del nombre del compuesto podemos obtener el número de oxidación de los átomos que lo forman. En la nomenclatura de Stock lo obtenemos mediante los números romanos que aparecen en su nombre y en la nomenclatura tradicional se deducen a partir de los prefijos y sufijos. La nomenclatura tradicional sigue siendo de amplio uso, lo mismo que la de Stock. La nomenclatura sistemática, recomendada por la IUPAC, es muy apreciada para nombrar los compuestos binarios.

Para resumir lo dicho, ofrecemos una serie de ejemplos en la tabla siguiente.

\begin{tabular}{|c|c|c|c|}
\hline Compuestos & N. sistemática & N. Stock & N. tradicional \\
\hline $\mathrm{SiO}_{2}$ & Dióxido de silicio & Óxido de silicio (IV) & Anhídrido silícico \\
\hline $\mathrm{Br}_{2} \mathrm{O}_{5}$ & Pentóxido de dibromo & Óxido de bromo (V) & Anhídrido brómico \\
\hline $\mathrm{SrO}$ & Óxido de estroncio & Óxido de estroncio & Óxido estróncico \\
\hline $\mathrm{H}_{2} \mathrm{SeO}_{3}$ & Trioxoseleniato de dihidrógeno & Seleniato(IV) de hidrógeno & Ácido selenioso \\
\hline $\mathrm{HClO}$ & Monoxoclorato de hidrógeno & Clorato (I) de hidrógeno & Ácido hipocloroso \\
\hline $\mathrm{HI}$ & Yoduro de hidrógeno & Yoduro de hidrógeno & Ácido yodhídrico \\
\hline $\mathrm{RbOH}$ & Hidróxido de rubidio & Hidróxido de rubidio & Hidróxido de rubidio \\
\hline $\mathrm{Pb}(\mathrm{OH})_{4}$ & Tetrahidróxido de plomo & Hidróxido de plomo (IV) & Hidróxido plúmbico \\
\hline $\mathrm{Fe}(\mathrm{OH})_{2}$ & Dihidróxido de hierro & Hidróxido de hierro (II) & Hidróxido ferroso \\
\hline $\mathrm{PCl}_{5}$ & Pentacloruro de fósforo & Cloruro de fósforo (V) & Cloruro fosfórico \\
\hline $\mathrm{Hg}\left(\mathrm{NO}_{2}\right)_{2}$ & Bis[dioxonitrato] de mercurio & Nitrito de mercurio (II) & Nitrito mercúrico \\
\hline $\mathrm{NaHCO}_{3}$ & Hidrogeno(trioxocarbonato) de & Hidrogenotrioxocarbonato & Carbonato ácido de \\
& sodio & (IV) de sodio & sodio \\
\hline
\end{tabular}

Tabla 3.2. Ejemplos de sistemas de nomenclatura 


\section{METODOLOGÍA}

\subsection{MUESTRA: LIBROS DE TEXTO SELECCIONADOS}

Vamos a estudiar el tema de nomenclatura y formulación en química inorgánica en una serie de manuales de $3^{0}$ y $4^{0}$ de Educación Secundaria Obligatoria pertenecientes a la asignatura Física y Química. Los textos han sido seleccionados de entre los más conocidos y utilizados. Son los siguientes:

\section{Libros analizados}

Libro 1: Arróspide, M.C., Manuel, M.M. (2008). Física y Química 4ํo ESO. Zaragoza: Edelvives

Libro 2: Balibrea, S., Reyes, M., Vílchez, J.M., Álvarez, A. y Sáez, A. (2008). Física y Química 4ํㅡㄹ ESO. Madrid: Anaya

Libro 3: Bullejos, J., Carmona, A., Hierrezuelo, J., Molina, E., Montero, A., Mozas, T., Ruiz, G., Sampedro, C., del Valle, V. (2008). Física y Química 4oESO. Granada: Elzevir Libro 4: Fidalgo, J.A., Fernández, M.R., Valdés, J. (2008). Física y Química $4^{\circ}$ ESO. León: Everest

Libro 5: Fontanet, A., Martínez, M.J. (2007). Física y Química 3o ESO. Barcelona: Vicens Vives

Libro 6: García, T. (2007). Física y Química 3ํES. Sevilla: Guadiel

Libro 7: Jiménez, R., Torres, P.M. (2007). Física y Química 3o ESO. Madrid: Bruño

Libro 8: Peña, A., Pozas, A., García, J.A., Rodríguez, A., Vasco, A.J. (2007). Física y Química 3ํㅡㅇ ESO. Madrid: McGraw-Hill

Libro 9: Piñar, I. (2010). Física y Química 3ํㅡㄹ. Madrid: Oxford University Press

Libro 10: Puente, J., Remacha, M. y Viguera, J.A. (2010). Física y Química 3ํ ESO. Madrid: Ediciones SM

Libro 11: Vidal, M.C., de la Prada, F., de Luis, J.L. (2007). Física y Química $3^{\circ}$ ESO. Madrid: Santillana

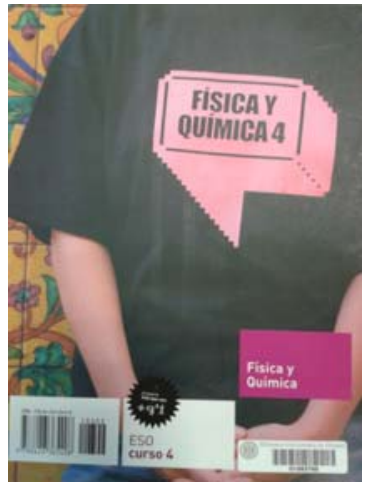

Libro 1

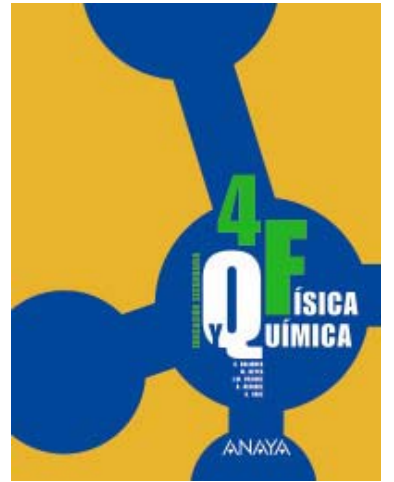

Libro 2

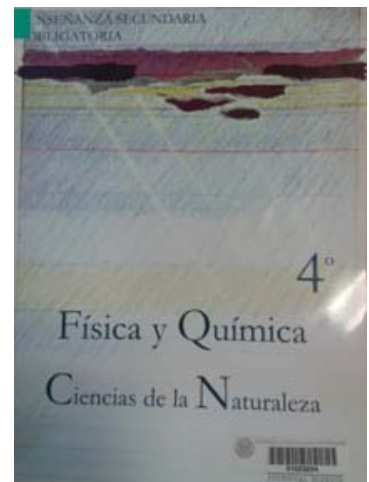

Libro 3 


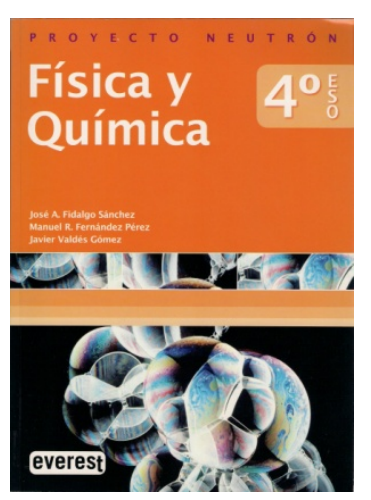

Libro 4

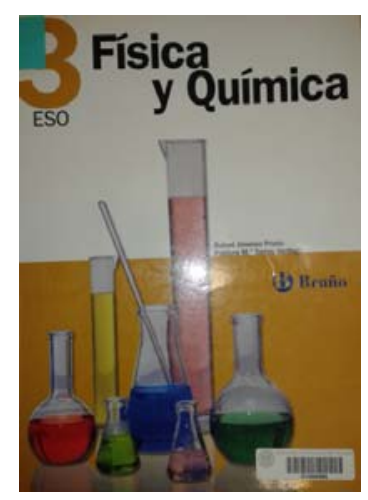

Libro 7

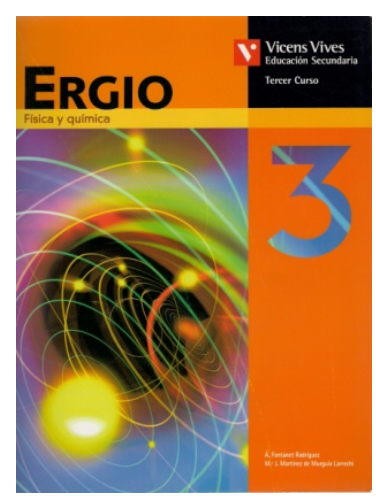

Libro5

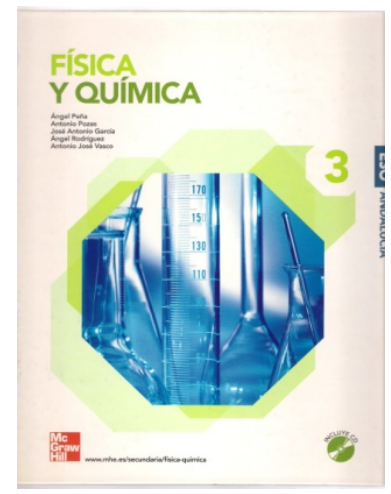

Libro 8

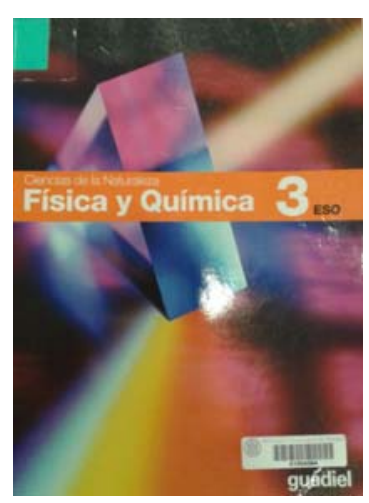

Libro 6

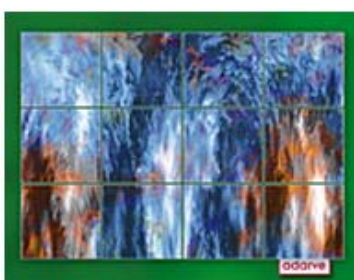

Física

y Química

$3^{\circ}$ ESO

Libro 9

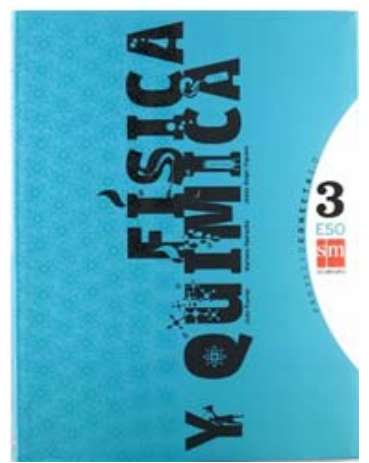

Libro 10

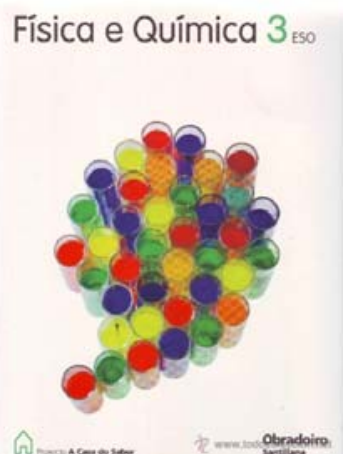

Libro 11

\subsection{TÓPICOS A CONSIDERAR EN EL ANÁLISIS}

En el capítulo 3, "Fundamentos" se estudiaron los conceptos de base en que se sustenta la formulación y nomenclatura de los compuestos químicos. Vamos a retomar estos tópicos para que nos sirvan de guía en el análisis de esta temática en los libros de texto. La tarea se llevará a cabo estudiando las modalidades de cada tópico que aparece en el desarrollo de la formulación en los manuales respectivos.

Como los tópicos presentan características muy diversas, a fin de ordenarlos se han agrupado en diversos ámbitos: situación en el manual, conceptos básicos que se utilizan, prerrequisitos exigidos para iniciar la formulación, procedimientos a 
seguir para formular, campo de compuestos que abarca la formulación propuesta y cuestiones de aplicación y sus tipos.

Mostramos brevemente cada uno, especificando los códigos que vamos a emplear en el protocolo de análisis (ap. 4.3, "claves de la tabla").

\section{Situación.}

Crs: Indicaremos si el libro analizado pertenece al tercer o cuarto curso de ESO, ya que son en ambos cursos donde puede comenzarse a estudiar el tema de la formulación.

Man: Examinaremos en qué parte del libro aparece desarrollada la formulación. Pueden ser las siguientes:

- Ps: La formulación está desarrollada en el texto principal del manual, formando parte de uno de los temas que incluye el libro. Se explica y expone la formulación completa dentro del mismo tema.

- Pf: La formulación se presenta también en el texto principal del manual, pero, a diferencia del caso anterior, ahora se expone dividida en dos o más temas del manual.

- Anx: La formulación se incluye al final del libro en un anexo (o varios), donde podemos encontrar todos los contenidos correspondientes.

- CD: Algunos manuales incluyen un CD-ROM para ampliar conocimientos. La formulación química puede aparecer desarrollada en ellos.

\section{Conceptos básicos.}

E/S: Constataremos si se hace una introducción sobre el concepto de elemento y además una presentación sobre sus símbolos correspondientes:

- E: En el manual encontramos una presentación sobre elemento químico.

- S: En el manual encontramos una presentación sobre símbolo químico.

M/nM: Observaremos si en los manuales aparece una diferenciación y clasificación clara entre metales y no metales.

F: Analizaremos si el manual se aporta una definición y explicación sobre el significado de la fórmula química de las sustancias.

F-N: Vamos a anotar qué manuales marcan la distinción entre formulación y nomenclatura y en cuáles esta distinción no se realiza. 
Comp: A la hora de enseñar la formulación de cada tipo de compuestos, podemos encontrar una breve definición sobre ellos. Anotaremos si encontramos esta definición en los manuales o no.

\section{Prerrequisitos.}

TP: Igualmente, nos fijaremos si el manual insiste en la tabla periódica dentro de la sección de formulación.

CfE: Vamos a observar si existen referencias a la configuración electrónica de los elementos en la sección de formulación.

Enl: Buscaremos si hay referencias en la sección de formulación a los diferentes tipos de enlace que podemos encontrar en los compuestos que se van a tratar.

\section{Procedimiento de formulación.}

Cb: Vamos a anotar sobre qué concepto de los siguientes se comienza a montar la formulación:

- v: Se utiliza el concepto de valencia.

- nox: Se utiliza el concepto de número de oxidación.

- "v": Se emplea el término de valencia dándole un significado de electrovalencia o de número de oxidación.

$\rightarrow$ F: Vamos a observar a partir de qué método se va a obtener la fórmula química:

- IntS: Intercambiando el número de oxidación o la valencia, seguido (si es el caso) de la posterior simplificación.

- FgS: A partir de la fórmula general y (si es el caso) su posterior simplificación.

- 0: Teniendo en cuenta que la sumatoria de los números de oxidación o de las "valencias" de los elementos que forman el compuesto debe ser igual a cero.

Anh $\rightarrow$ Tendremos en cuenta si para obtener la fórmula del oxácido, el método que se propone es a partir del anhídrido correspondiente.

\section{Ámbito de formulación.}

Clas: Vamos a observar cuál es el criterio de clasificación que emplean los manuales para ordenar los diferentes tipos de compuestos que se van a enseñar:

- An: Si emplean un criterio analítico. 
- TC: Si clasifican por tipo de compuestos.

Bin: Anotaremos si en los manuales sólo se explican los compuestos binarios o no.

MH: Indicaremos qué manuales consideran la formulación de hidruros metálicos.

Ntip: Cuáles son los tipos de nomenclatura que propone cada manual:

- T: Se da la nomenclatura tradicional

- St: Se da la nomenclatura de Stock

- S: Se da la nomenclatura sistemática

- TStS: Se dan las tres anteriores.

\section{Cuestiones de aplicación.}

Cuest: Evaluaremos de qué tipo son las cuestiones de aplicación que aparecen en los libros de texto analizados:

- $\quad \mathrm{N} \rightarrow \mathrm{F}$ : Los ejercicios que encontramos en el manual son ejercicios donde se aporta la nomenclatura y el alumno debe dar la fórmula.

- $\quad \mathrm{F} \rightarrow \mathrm{N}$ : Los ejercicios que encontramos en el manual son ejercicios donde se aporta la fórmula química y el alumno debe nombrar el compuesto.

- NQF: En un mismo ejercicio se debe de formular y nombrar los compuestos.

- $\mathrm{F}+\mathrm{N}$ : Los ejercicios que propone el manual son de los dos primeros tipos, indistintamente.

\subsection{PROTOCOLO DE ANÁLISIS DE LIBROS DE TEXTO}

Para poder llevar a cabo el estudio de la metodología seguida por los manuales de Educación Secundaria Obligatoria con respecto al tema que estamos tratando se analizarán una serie de conceptos y procedimientos en los manuales estudiados para, posteriormente, extraer conclusiones sobre su metodología. 
Los conceptos y procedimientos que vamos a tener en cuenta se resumen en la siguiente tabla:

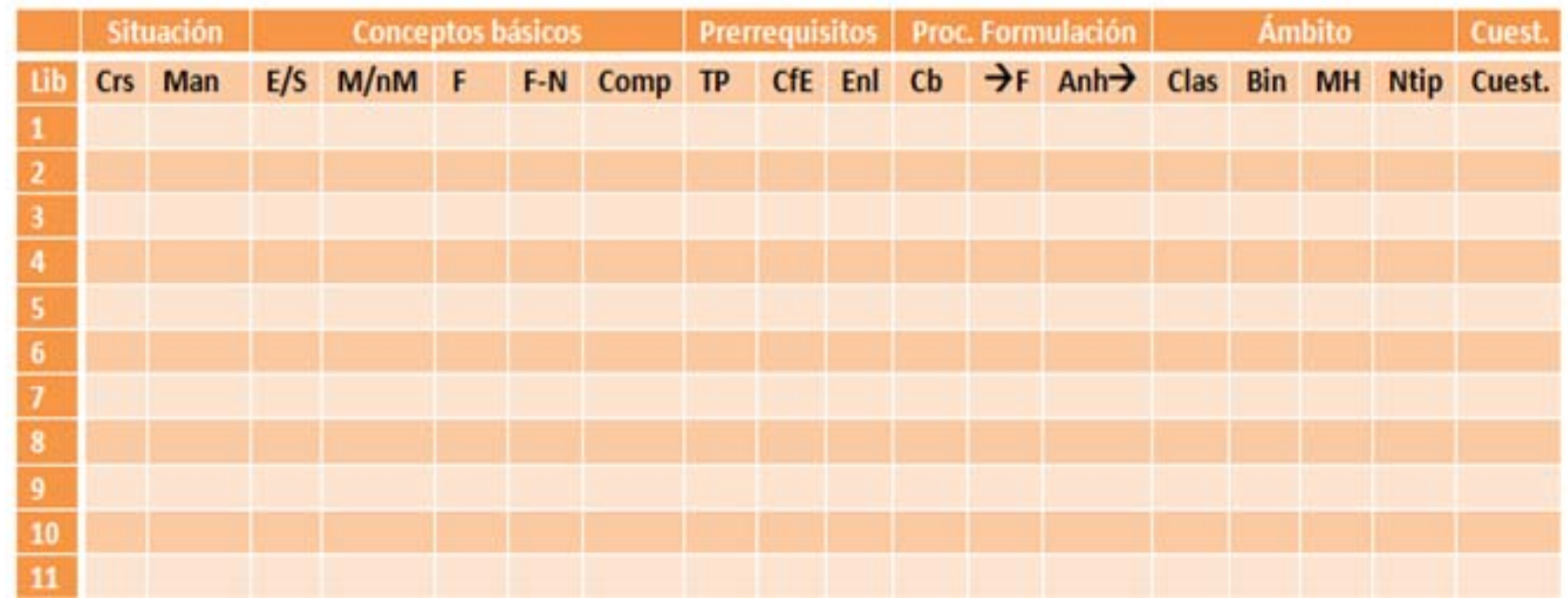

Tabla 4.1. Resumen del protocolo de análisis de libros de texto

\section{Claves de la tabla}

\section{Crs: Curso \\ Man: Manual}

Ps: en el texto principal todo seguido Pf: id. fraccionado

Anx: en anexos

CD: en CD-ROM

E/S: Elemento/Símbolo

M/nM: División entre metal y no metal

F: Significado de la fórmula

F-N: Diferencia entre formulación y nomenclatura

Comp: Definición de tipo de compuestos

TP: Tabla periódica

CfE: Configuración electrónica

Enl: Tipos de enlace

Cb: Concepto de base

v: valencia

nox: $\mathrm{n}^{\mathrm{o}}$ oxidación

"v": con significado de electroval./nox $\rightarrow$ F: Deducción de la fórmula IntS: intercambio de val./nox+simplif. FgS: form.gen.+simplif.

0: $\Sigma$ nox/"val" $=0$

Anh $\rightarrow$ : Se parte del anhídrido (para oxácidos)

Clas: Criterio de clasificación

An: analítico

TC: tipo de compuestos

Bin: Sólo compuestos binarios

MH: Hidruros metálicos

Ntip: Tipos de nomenclatura a enseñar

T: tradicional

St: Stock

S: sistemática

TStS: elementos de las tres

Cuest: Cuestiones de aplicación

$\mathrm{N} \rightarrow \mathrm{F}$ : se da $\mathrm{N}$ y se pide $\mathrm{F}$

$\mathrm{F} \rightarrow \mathrm{N}$ : al contrario

NQF: se piden las dos (al mismo $t$ )

$\mathrm{F}+\mathrm{N}$ : existen tanto $\mathrm{N} \rightarrow \mathrm{F}$ y $\mathrm{F} \rightarrow \mathrm{N}$ 


\section{Resultados. Análisis de resultados}

\subsection{CARACTERÍSTICAS DE LA FORMULACIÓN QUÍMICA EN LOS MANUALES}

Para poder obtener resultados sobre el estudio de las propuestas de enseñanza de la formulación química en los manuales de $3^{\text {o }}$ y $4^{\text {o }}$ de E.S.O. ha sido analizada la parte correspondiente a la formulación y nomenclatura inorgánicas en una serie de manuales editados a partir de la implantación del Real Decreto 1631/2006, del 29 de diciembre, el cual corresponde a la última ordenación curricular de la Educación Secundaria Obligatoria.

\section{Editoriales}

Libro 1: Edelvives

Libro 2: Anaya

Libro 3: Elzevir

Libro 4: Everest

Libro 5: Vicens Vives

Libro 6: Guadiel
Libro 7: Bruño

Libro 8: McGraw-Hill

Libro 9: Oxford

Libro 10: Ediciones SM

Libro11: Santillana

La formulación que presentan los manuales anteriores se ha analizado aplicando el protocolo que fue dado en el apartado 4.3. Los resultados obtenidos se recogen esquemáticamente en la Tabla 5.1

\begin{tabular}{|c|c|c|c|c|c|c|c|c|c|c|c|c|c|c|c|c|c|c|}
\hline & \multicolumn{2}{|c|}{ Situación } & \multicolumn{5}{|c|}{ Conceptos básicos } & \multicolumn{3}{|c|}{ Prerrequisitos } & \multicolumn{3}{|c|}{ Proc. Formulación } & \multicolumn{4}{|c|}{ Ámbito } & \multirow{2}{*}{$\begin{array}{l}\text { Cuest. } \\
\text { Cuest. }\end{array}$} \\
\hline Lib & Crs & Man & $E / S$ & $\mathrm{M} / \mathrm{nM}$ & $\mathbf{F}$ & $\mathrm{F}-\mathrm{N}$ & Comp & TP & CfE & Enl & $\mathrm{Cb}$ & $\rightarrow_{\mathrm{F}}$ & $\mathrm{Anh} \rightarrow$ & Clas & Bin & MH & Ntip & \\
\hline 1 & 49 & Anx & $\mathrm{S}$ & $\checkmark$ & $\checkmark$ & $\checkmark$ & $\checkmark$ & - & - & - & $\mathrm{v}$ & Ints & $\checkmark$ & A & - & $\checkmark$ & TStS & $\mathrm{F}+\mathrm{N}$ \\
\hline 2 & 49 & $\mathrm{Pf}+\mathrm{CD}$ & - & $\checkmark$ & - & $\checkmark$ & $\checkmark$ & $\checkmark$ & - & - & "v" & Ints & $\checkmark$ & TC & - & $\checkmark$ & $\mathrm{T}+\mathrm{St}$ & $\mathrm{F}+\mathrm{N}$ \\
\hline 3 & 49 & Ps & $E+S$ & $\checkmark$ & $\checkmark$ & - & $\checkmark$ & - & - & - & "v" & Ints & - & A & - & - & TStS & $\mathrm{F}+\mathrm{N}$ \\
\hline 4 & 49 & Anx & $S$ & - & $\checkmark$ & - & $\checkmark$ & - & - & - & nox & $\mathrm{FgS}$ & $\checkmark$ & $\mathrm{TC}$ & - & $\checkmark$ & TStS & $\mathrm{F}+\mathrm{N}$ \\
\hline 5 & 30 & Ps & $\mathrm{E}$ & - & $\checkmark$ & - & $\checkmark$ & - & - & - & - & - & - & $\mathrm{TC}$ & $\checkmark$ & $\checkmark$ & St & $\mathrm{F}+\mathrm{N}$ \\
\hline 6 & 30 & Anx & - & $\checkmark$ & - & $\checkmark$ & $\checkmark$ & - & - & - & nox & Ints & - & A & - & $\checkmark$ & TStS & $\mathrm{N} \leftrightarrow \mathrm{F}$ \\
\hline 7 & 30 & Anx & $E$ & $\checkmark$ & $\checkmark$ & $\checkmark$ & $\checkmark$ & $\checkmark$ & - & - & nox & Ints & $\checkmark$ & A & - & $\checkmark$ & TStS & $\mathrm{F} \rightarrow \mathrm{N}$ \\
\hline 8 & 39 & Anx & $E+S$ & $\checkmark$ & $\checkmark$ & - & $\checkmark$ & - & - & - & nox & $\mathrm{FgS}$ & $\checkmark$ & A & - & $\checkmark$ & TStS & $\mathrm{F}+\mathrm{N}$ \\
\hline 9 & 30 & Anx & $E$ & $\checkmark$ & $\checkmark$ & $\checkmark$ & $\checkmark$ & $\checkmark$ & - & - & "v" & Ints & $\checkmark$ & A & - & $\checkmark$ & TStS & $\mathrm{F}+\mathrm{N}$ \\
\hline 10 & 39 & Anx & $\mathrm{E}$ & $\checkmark$ & - & $\checkmark$ & $\checkmark$ & $\checkmark$ & - & - & nox & Ints & $\checkmark$ & A & - & $\checkmark$ & TStS & $\mathrm{N} \leftrightarrow \mathrm{F}$ \\
\hline 11 & 30 & Anx & - & $\checkmark$ & - & $\checkmark$ & $\checkmark$ & $\checkmark$ & $\checkmark$ & - & $\mathrm{v}$ & Ints & - & A & - & $\checkmark$ & TStS & $\mathrm{F}+\mathrm{N}$ \\
\hline
\end{tabular}

Tabla 5.1. Resultados del análisis de diversos libros de texto (3ํ-ํํㅡㄴ 


\section{Claves de la tabla}

\begin{tabular}{|l|l|}
\hline Crs: Curso & $\rightarrow$ F: Deducción de la fórmula \\
Man: Manual & IntS: intercambio de \\
Ps: en el texto principal todo & val./nox+simplif. \\
seguido Pf: id. fraccionado & FgS: form.gen.+simplif. \\
Anx: en anexos & $0: \sum$ nox/"val"= \\
CD: en CD-ROM & Anh $\rightarrow$ : Se parte del anhídrido (para \\
E/S: Elemento/Símbolo & oxácidos) \\
M/nM: División entre metal y no & Clas: Criterio de clasificación \\
metal & An: analítico \\
F: Significado de la fórmula & TC: tipo de compuestos \\
F-N: Diferencia entre formulación y & Bin: Sólo compuestos binarios \\
nomenclatura & MH: Hidruros metálicos \\
Comp: Definición de tipo de & Ntip: Tipos de nomenclatura a \\
compuestos & enseñar \\
TP: Tabla periódica & T: tradicional \\
CfE: Configuración electrónica & St: Stock \\
Enl: Tipos de enlace & S: sistemática \\
Cb: Concepto de base & TStS: elementos de las tres \\
v: valencia & Cuest: Cuestiones de aplicación \\
nox: $n$ ooxidación & N $\rightarrow$ F: se da $N$ y se pide F \\
"v": con significado de & F $\rightarrow$ N: al contrario \\
electroval./nox & N⿴囗十: se piden las dos (al mismo t) \\
& F+N: existen tanto N $\rightarrow \mathrm{F}$ y F $\rightarrow \mathrm{N}$ \\
\hline
\end{tabular}

\subsection{ANÁLISIS DE RESULTADOS}

A partir del estudio realizado sobre formulación química en los manuales de ESO, hemos obtenido los resultados que se recogen en la Tabla 5.1. Vamos a analizar los resultados obtenidos en cada uno de los tópicos considerados.

\section{Presentación de la formulación.}

Como vimos anteriormente el Real Decreto de Enseñanzas Mínimas incluye la formulación en el curso $4^{\circ}$ de ESO, señalando, además, que va a limitarse a los compuestos inorgánicos binarios. Sin embargo en la mayor parte de los manuales aparece en el curso $3^{\circ}$ (Tabla 5.1, col. "Crs"). En cuanto a contenidos presentan prácticamente el mismo independientemente de que el curso sea $3^{\circ}$ o $4^{\circ} \stackrel{\text {. Se }}{ }$ constata, además, que hacen caso omiso a tratar sólo los compuestos binarios y ofrecen un panorama completo de la formulación.

En cuanto a la situación en el manual, la formulación aparece desarrollada como anexo en la mayor parte de los casos (col. "Man"), (Anexo III y Anexo IV). Esto es casi general cuando se incluye en $3^{\circ}$ de ESO. Pero en los manuales de $4^{\circ}$ o 
examinados aparece tanto en anexo, como en el texto principal, ya sea seguido o fragmentado, e incluso podemos encontrar más información en CD-ROM.

\section{Conceptos básicos.}

Puesto que la formulación está montada sobre el concepto de elemento químico y su representación abreviada que es el símbolo es conveniente que los manuales definan y aclaren previamente el significado de estos dos términos. A este respecto encontramos que la mayoría de los manuales hacen referencia a los elementos y muy pocos añaden una pequeña introducción sobre los símbolos (col. "E/S”).

Saber que hay dos tipos de elementos, metales y no metales supone tener una clasificación inicial de los elementos. Esto es muy necesario para iniciarse a la formulación, pues de esta manera se facilita el acceso a la formulación a los alumnos que pueden distinguir más fácilmente qué tipo de compuestos formarán los elementos. En la mayoría de los manuales analizados se hace esta distinción (col. "M/nM").

El significado de la fórmula es esencial para que el alumnado entienda de qué estamos hablando y cuál es el motivo de la formulación. Si el alumnado no entiende qué nos quiere decir la fórmula química de un compuesto entonces este tema será aprendido de manera mecánica y memorística, aplicando las normas y sin comprender la composición cualitativa y cuantitativa de las sustancias. Los manuales se hacen eco de este hecho, por ello la mayoría explica el significado de la fórmula química (col. "F”), (Anexo III).

En la mayor parte de los manuales analizados se marca la diferencia entre formulación y nomenclatura (col. "F-N"), (Anexo I). Se hace hincapié en que una cosa es la representación simbólica de una sustancia y otra el nombre de la misma.

A la hora de clasificar y formular los diferentes compuestos se aporta en todos y cada uno de los manuales la definición de cada tipo compuesto, describiendo su composición general y el tipo de elementos que lo forman (col. "Comp").

\section{Prerrequisitos.}

Una buena parte de los manuales considera la tabla periódica como un prerrequisito indispensable para aprender a formular. Lo que se ve habitualmente no es exigir un conocimiento completo de ella, pero sí de los grupos principales (Anexo III y Anexo IV). Otros manuales, aún incluyéndola como contenido, no le atribuyen el papel de prerrequisito (col. "TP").

La configuración electrónica de los átomos nos muestra la distribución de sus electrones en los distintos niveles, y cuyo interés para el químico reside especialmente en los más externos. Sólo un manual considera estos conocimientos indispensables para iniciar la formulación (col. “CfE”), (Anexo IV). 
En cuanto al papel del tipo de enlace de las distintas sustancias (col. "Enl") como prerrequisito para la formulación, no aparece en ningún manual, si bien es cierto que el tema figura entre los contenidos especificados por el Real Decreto para el cuarto curso.

\section{Procedimiento de formulación.}

Conceptos de base son los términos de valencia y número de oxidación. Unos manuales emplean el concepto de valencia para la enseñanza de la formulación, otros el número de oxidación (Anexo I), e incluso algunos emplean un concepto intermedio entre ambos términos (col. "Cb"). La mayoría de nuestros manuales utilizan el número de oxidación como concepto base.

Aparecen también varios métodos para conseguir la fórmula química de un compuesto (col. " $\rightarrow F^{\prime}$ ). El primero y más extendido es a partir del intercambio de valencias o de números de oxidación entre los elementos que constituyen el compuesto, seguido de una posterior simplificación de subíndices, si fuese posible (Anexo I y Anexo II). El segundo consiste en formular partiendo de la fórmula general de cada compuesto. Por último, un tercer método utilizado consiste en tantear con los subíndices para intentar que la suma algebraica, teniendo en cuenta los números de oxidación, sea igual a cero (no vale, claro está, para los iones).

En el caso particular de los oxácidos, el método más utilizado para alcanzar su fórmula es partir del anhídrido correspondiente y sumar las oportunas moléculas de agua (col. "Anh $\rightarrow$ "), sin excluir, a veces, el dividir por 2 (Anexo III).

\section{Ámbito de la formulación.}

El criterio de clasificación de los compuestos que se abarcan (col. "Clas") puede ser de dos tipos: una clasificación de tipo analítica (compuestos binarios, ternarios...), (Anexo I, Anexo III y Anexo IV) y otra en función del tipo de compuestos (óxidos, ácidos...). Hemos observado que la más habitual es la clasificación mediante el criterio analítico.

Hemos analizado igualmente si en los manuales se abarca la totalidad de tipos de compuestos, o por el contrario sólo se consideran los compuestos binarios (col. "Bin"), como propone el RD., que son desde luego los más simples para comenzar el aprendizaje de la formulación. Hemos concluido que esto no es lo que suele pasar y la mayoría de los manuales abarcan más tipos de compuestos, no sólo los binarios independientemente de que pertenezcan al tercer o cuarto curso.

Los hidruros metálicos son compuestos que aparecen en casi todos los manuales (Anexo I, Anexo III y Anexo IV). Hemos seguido su presencia (col. "MH") para corroborar que es la clasificación la que conduce a presentar este tipo de compuestos, que no son muy usuales en el trabajo científico. 
Los tipos de nomenclatura que se exponen en los manuales también han sido analizados (col. "NTip"). Se proponen los tres tipos de nomenclatura aunque algunas estén en desuso. Excepto en dos de los once manuales estudiados, se ilustran y se explican los tres tipos de nomenclatura (sistemática, de Stock y tradicional), (Anexo I, Anexo III y Anexo IV).

\section{Cuestiones de aplicación.}

Las cuestiones de aplicación (col. "Cuest.") son un tópico realmente importante a considerar. En la mayoría de los manuales aparecen dos tipos de ejercicios que consisten, o bien en que a partir de la fórmula química el alumno proponga el nombre de la sustancia, o bien que a partir de la nomenclatura escriba la fórmula (Anexo IV).

\subsection{DISCUSIÓN DE RESULTADOS}

Pasamos ahora a discutir e interpretar los resultados obtenidos (ap. 5.1) y analizados anteriormente (ap. 5.2) siguiendo uno por uno los tópicos considerados en el análisis.

\section{Presentación de la formulación.}

La aparición mayoritaria de la formulación en $3^{\circ}$ se comprende porque los autores y quizás la mayor parte de los profesores ven conveniente empezar a utilizarla en $3^{\text {o }}$, donde hay temas (Bloque 4) dedicado a las reacciones químicas. Por otra parte, puede que la intención del Ministerio al limitar la formulación a compuestos binarios fuera evitar exposiciones sobrecargadas, lo cual es positivo. Pero no ha acertado con esta medida (no deberían estudiarse entonces $\mathrm{H}_{2} \mathrm{SO}_{4}, \mathrm{CaCO}_{3}$, etc.). Es, pues, gratificante que los manuales hayan ignorado algo tan poco procedente.

En cuanto a la situación de la formulación como anexo, la diferencia entre los manuales de $3^{\circ}$ y de $4^{\circ}$ puede deberse a que en $3^{\circ}$ no aparece la formulación en el RD y por ello se adopta incluirlo como anexo.

\section{Conceptos básicos}

Es imprescindible para formular tener una conciencia clara de lo que es un elemento y conocer el símbolo de los que van a emplearse. El hecho de que en la mayoría de los manuales no se desarrolla este último puede deberse a que se puede considerar prescindible hacer una introducción al símbolo químico, pasando directamente a su uso en la formulación. 
Es positivo también marcar la diferencia entre metales y no metales, porque puede ayudar bastante a comprender la formación de compuestos y las reglas de formulación.

Por otra parte, saber el significado que encierra una fórmula química es la llave para iniciarse con buen pie en el aprendizaje de formulación. En consecuencia no es admisible que más de la tercera parte de los manuales silencien esta cuestión.

Tampoco es recomendable encontrar la misma situación respecto a diferenciar los términos nomenclatura y formulación, sabiendo que muchos alumnos los hacen sinónimos.

En cambio mejor comportamiento manifiestan los manuales exponiendo claramente los tipos de compuestos más esenciales, por su utilidad a la hora de formular los casos concretos.

\section{Prerrequisitos}

Una idea muy arraigada entre una considerable parte del profesorado es la importancia de la tabla periódica para la formulación. Las razones esgrimidas es que puede ayudar a asimilar y aprender las valencias o los números de oxidación de los elementos, marca la diferencia entre metales y no metales y resalta las semejanzas entre grupos de elementos. Esto es cierto, pero debemos también preguntarnos como profesores si el esfuerzo que supone asimilar una serie de conceptos de alto nivel de abstracción merece la pena. Sin contar los efectos emocionales y vocacionales adversos que puede provocar en alumnos que comienzan a dar sus primeros pasos en ciencias.

Lo mismo podría decirse respecto a las configuraciones electrónicas. Entenderlas y saber desarrollarlas puede ser más efectivo en cursos superiores, una vez que la formulación esté asimilada. Quizás por este motivo los manuales estudiados no han insistido en ello.

En cuanto al enlace, es interesante conocer el tipo de enlace que forman los compuestos, ya que éste marca las propiedades, pero estas diferencias no ayudan de modo apreciable la iniciación al aprendizaje de la formulación. En los manuales estudiados no aparecen conexiones del enlace con la formulación.

Por todas estas razones, podemos encontrar profesores que rechazan la idea de la tabla periódica como prerrequisito de la formulación y abogan por una enseñanza mínima de esta, al margen de la tabla periódica, las configuraciones electrónicas y el enlace químico.

\section{Procedimiento de formulación.}

En la mayoría de los manuales hemos encontrado que la formulación es tratada a través del concepto de número de oxidación. Esto puede ser debido a que muchos 
autores creen que el uso del número de oxidación puede ser el más exacto e implicar menos errores, pese a su elevado nivel teórico.

El método más común para deducir la fórmula de un compuesto es a partir del intercambio de sus valencias o números de oxidación, seguido por la operación de simplificación. En compuestos no binarios el intercambio se entiende entre iones o grupos. El método es aparentemente sencillo, pero su fundamento químico no aparece en ningún manual. Se han encontrado algún texto que para llegar a la fórmula de un compuesto parte de la fórmula general del tipo al que pertenece. Este método es más dificultoso ya que el alumno debe aprenderse memorísticamente las fórmulas generales, mientras que en el método anterior sólo debe aprender los números de oxidación o las valencias, empleando un método aritmético que los conduce a la fórmula. No hemos encontrado ningún manual que emplee la tercera forma antes descrita para deducir la fórmula, método más engorroso, ya que el alumno debe ir comprobando mediante cálculos matemáticos si cada fórmula es correcta, con lo cual es más fácil cometer errores.

Alcanzar la fórmula de los oxoácidos partiendo de su anhídrido es el método más empleado en los manuales. Al parecer es un método sencillo, pero esconde complicaciones (¿cuántas moléculas de agua añadir?) y no se libra, muchas veces de la etapa de simplificación.

\section{Ámbito de la formulación.}

El criterio analítico es el criterio de clasificación más usual empleado en los manuales y es la guía para marcar el ámbito de la formulación. Es posible que este criterio predomine porque la formulación de compuestos no binarios entraña algo más de dificultad (discutible: pensemos en el caso de los hidróxidos). Así que en primer lugar se enseña a formular los compuestos más simples, que son los compuestos binarios, para después llegar a los más complejos.

En la gran mayoría de los manuales analizados también se describe una variedad amplia de compuestos, no sólo los compuestos binarios, como se podría pensar por empezar con menor dificultad o tal y como se describe en el RD. Los manuales consideran que debe abarcarse el ámbito completo de la formulación.

La enseñanza de los hidruros metálicos está presente en prácticamente todos los manuales consultados. La presencia de estos compuestos corrobora que es la clasificación la que impone estudiarlos. Aunque son compuestos poco frecuentes en el trabajo científico, según los manuales, los alumnos deben conocerlos y saber formularlos, por aquello de que alguna vez puedan encontrarse con alguna referencia a los mismos.

No todos los sistemas de nomenclatura se recomiendan hoy en día, pero en la práctica habitual de laboratorio se sigue empleando la nomenclatura tradicional. Los tres sistemas comentados son enseñados en los manuales, quizás convencidos, 
lo mismo que parte del profesorado, que es lo mejor para que los alumnos no tengan dificultades a la hora de encontrarse con cualquiera de ellos.

\section{Cuestiones de aplicación.}

Lo habitual es encontrar ejercicios en los que se da el nombre y se pide la fórmula o viceversa. Este tipo de ejercicios son recomendables por su sencillez para empezar. Pero la oferta de cuestiones no debe limitarse a ellos. Lo ideal es, como aparece en pocos manuales, encontrar al menos algún ejercicio en el que se pida tanto la fórmula como la nomenclatura de las sustancias (Anexo I). 
Las principales tareas que hemos emprendido en el transcurso de nuestro trabajo de investigación ya se fijaron en el inicio del mismo, guiado por los objetivos (ap. 2.2). En el capítulo 2 nos planteamos una serie de interrogantes (ap. 2.1) a los que la investigación debería responder. Esto ha sido debidamente cumplido. Ya estamos en disposición de suministrar una respuesta bien fundamentada a aquellas cuestiones planteadas.

Ahora, como cierre de nuestra investigación, vamos a exponer las conclusiones generales que se infieren del trabajo realizado. Todas están vinculadas con los objetivos inicialmente propuestos. Adelantándonos a la exposición de las conclusiones, mostramos en la Tabla 6.1 la relación entre éstas y los objetivos del trabajo. Recordando estos objetivos (ap. 2.2) y considerando las conclusiones que siguen, no es difícil de comprender las conexiones establecidas en la tabla y, por ello, no merecen más comentario.

\begin{tabular}{|c|c|c|c|c|c|c|}
\hline Objetivos & 1 & 2 & 3 & 4 & 5 & 6 \\
\hline Conclusiones & 1 & 3 & 2 & $4 / 6$ & 5 & 7 \\
\hline
\end{tabular}

Tabla 6.1. Adscripción de las conclusiones a los objetivos de la investigación

Las conclusiones principales del trabajo de investigación llevado a cabo son:

1. Se han señalado los elementos conceptuales y procedimentales que intervienen normalmente en la enseñanza de la formulación a nivel inicial.

2. En base a lo anterior se ha puesto a punto un protocolo de análisis de libros de texto que se ha aplicado a una muestra de manuales de $3^{\circ}$ y $4^{\circ}$.

3. La mayor parte de los manuales estudiados presentan la formulación en $3^{\mathrm{o}} \mathrm{y}$ suelen exponerla en un anexo. Ignoran lo establecido en el Decreto de Mínimos de la ESO, que incluye la formulación en $4^{\circ}$ y limitada, además, a compuestos binarios.

4. Raro es el manual que no explica el significado de elemento químico, la distinción metales/ no metales, el significado de la fórmula, la diferencia entre formulación y nomenclatura, y la descripción de cada tipo de compuesto.

5. En cuanto a procedimientos de formulación, suelen basarse en los conceptos de valencia o número de oxidación. El método más extendido para conseguir la fórmula de un compuesto es el de intercambio de valencias o de nox, seguido de 
una posterior simplificación de subíndices, si es posible. En el caso particular de los oxácidos es muy utilizado el método de partir del anhídrido y sumar las oportunas moléculas de agua.

6. Los manuales, con alguna excepción, presentan y aplican los tres tipos de nomenclatura, sistemática, de Stock y tradicional. Las cuestiones de aplicación que suelen proponerse son las de partir del nombre y averiguar la fórmula, o viceversa.

7. Más de la tercera parte de los manuales silencian en su planteamiento inicial el significado de una fórmula. La mitad considera la tabla periódica como prerrequisito indispensable para aprender a formular. Casi todos proponen como procedimiento de formulación la aplicación mecánica de las reglas del intercambio y de la divisibilidad.

\section{Perspectivas futuras}

El trabajo expuesto podría desarrollarse posteriormente investigando con más detenimiento los elementos que han aparecido como más inadecuados y elaborando una propuesta didáctica alternativa de la enseñanza de la formulación. 


\section{BIBLIOGRAFÍA}

Ben-Zvi, R., Eylon, B. y Silberstein, J. (1988). Theories, principles and laws. Education in Chemistry, 5, 89-92.

Chamizo, J.A., Nieto, E. y Sosa P. (2004). La enseñanza de la química. Tercera parte. Evaluación de los conocimientos de química desde secundaria hasta licenciatura. Educación química, 15(2), 60-65.

De Jong 0. (1996). La investigación activa como herramienta para mejorar la enseñanza de la química: nuevos enfoques. Investigación y experiencias didácticas, 14(3), 279-288.

Driver, R. (1985). Beyond Appearances: The Conservation of Matter under Physical and Chemical Transformations. En R. Driver, E. Guesne y A. Tiberghien (Eds.) Children's Ideas in Science, pp. 145-169. Milton Keynes: Open University Press.

Fernández-González, M. (2008). Ciencias para el mundo contemporáneo. Algunas reflexiones didácticas. Eureka, revista sobre Enseñanza y Divulgación de las Ciencias, 5(2), 185-199.

Gabel, D. (1998). The complexity of chemistry and implications for teaching. En B.J. Fraser y K.G. Tobin (Eds.), International handbook of science education, pp. 233248. Boston: Kluwer.

García, J.A. y Teijón, J.M. (1993). Formulación y nomenclatura de química inorgánica. Normas IUPAC. Madrid: Tébar Flores.

Griffiths, A.K. y Preston, K.R. (1992). Grade-12 students' misconceptions relating to fundamental characteristics of atoms and molecules. Journal of Research in Science Teaching, 29(6), 611-628.

Hernández, J. y Palacín, L. (1993). La formulación en el nuevo currículum de la química. Comunicación, lenguaje y educación, 19-20, 101-107.

Hierrezuelo, J. y Montero, A. (1991). La ciencia de los alumnos. Vélez-Málaga: Elzevir

Josa, S. (1995). Guía básica de formulación y nomenclatura. Química inorgánica y orgánica para estudiantes de Secundaria. Barcelona: Edunsa.

Jiménez-Aleixandre, M.P. (2000). Modelos didácticos. En F.J. Perales y P. Cañal (Dirs.) Didáctica de las Ciencias Experimentales, pp. 165-186. Alcoy: Marfil.

Johnstone, A.H. (1993). The development of chemistry teaching. Journal of Chemical Education, 70(9), 701-705. 
Latorre, M. (1999). Formulación y nomenclatura en química inorgánica. Normas de la IUPAC. Zaragoza: Edelvives.

Montiano, M.J. (2010). Didáctica de la química a través de los juegos. Temas para la educación, 11(66), 1-10.

Muñoz, J.M. (2010). Juegos Educativos. FyQ formulación. Eureka, revista sobre Enseñanza y Divulgación de las Ciencias, 7(2), 559-565.

Ministerio de Educación y Ciencia (2006) Real Decreto 1631/2006 de Enseñanzas Mínimas de la ESO. BOE de 5-1-07, 677-773.

Nakhleh, M.B. (1992). Why Some Students Don't Learn Chemistry. Journal of Chemical Education, 69(3), 191-196.

Parchmann, I. (2011). La enseñanza de la química y el año internacional de la química. Oportunidades para mejorar la comprensión y el interés de los estudiantes. Alambique. Didáctica de las Ciencias Experimentales, 69, 8-20.

Peterson, W. R. (1987). Formulación y nomenclatura en química inorgánica según la normativa de la IUPAC. Barcelona: Edunsa.

Rocard, M., Csermely, P., Jorde, D., Lenzen, D., Walwerg-Henriksson, H. y Hemmo, V. (2008). Enseñanza de las ciencias ahora: Una nueva pedagogía para el fututo de Europa. Alambique. Didáctica de las Ciencias Experimentales, 55, 104-117.

Rodrigo, M.J. y Cubero, R. (2000). Constructivismo y enseñanza de las ciencias. En F.J. Perales y P. Cañal (Dirs.) Didáctica de las Ciencias Experimentales, pp. 85-107. Alcoy: Marfil.

Solbes, J., Montserrat, R. y Furió, C. (2007). El desinterés del alumnado hacia el aprendizaje de la ciencia: implicaciones en su enseñanza. Didáctica de las ciencias experimentales y sociales, (21) 91-117.

Taskin, V. \& Bernholt, S. (2012). Students' Understanding of Chemical Formulae: A review of empirical research. International Journal of Science Education, Consultado en http://dx.doi.org/10.1080/09500693.2012.744492

\section{Manuales analizados:}

Arróspide, M.C. y Manuel, M.M. (2008). Física y Química 4º ESO. Zaragoza: Edelvives

Balibrea, S., Reyes, M., Vílchez, J.M., Álvarez, A. y Sáez, A. (2008). Física y Química 4o ESO. Madrid: Anaya 
Bullejos, J., Carmona, A., Hierrezuelo, J., Molina, E., Montero, A., Mozas, T., Ruiz, G., Sampedro, C. y del Valle, V. (2008). Física y Química 4ํo ESO. Granada: Elzevir

Fidalgo, J.A., Fernández, M.R. y Valdés, J. (2008). Física y Química 4o ESO. León: Everest

Fontanet, A., Martínez, M.J. (2007). Física y Química 3ํo ESO. Barcelona: Vicens Vives García, T. (2007). Física y Química 3º ESO. Sevilla: Guadiel

Jiménez, R. y Torres, P.M. (2007). Física y Química 3º ESO. Madrid: Bruño

Peña, A., Pozas, A., García, J.A., Rodríguez, A. y Vasco, A.J. (2007). Física y Química 3º ESO. Madrid: McGraw-Hill

Piñar, I. (2010). Física y Química 3ํESO. Madrid: Oxford University Press

Puente, J., Remacha, M. y Viguera, J.A. (2010). Física y Química 3ํE. ESO. Madrid: Ediciones SM

Vidal, M.C., de la Prada, F. y de Luis, J.L. (2007). Física y Química 3o ESO. Madrid: Santillana 


\title{
MUESTRA REPRESENTATIVA DE LOS LIBROS DE TEXTO
}

\author{
ANEXO I: García, T. (2007). Física y Química 3ํo ESO. Sevilla: Guadiel
}

\section{Formulación y nomenclatura de compuestos inorgánicos}

Para formular un compuesto es útil suponer que está constituido por iones, aunque sabemos que no siempre es asi. La carga imaginaria, positiva o negativa, que se asigna a cada elemento en un compuesto rocibe el nombre de nú. mero de oxidación.

Siempre se debe cumplir que la suma de los números de oxidación do todos los átcmos de los elementos que in. tervienen en un compuesto debe ser cero.

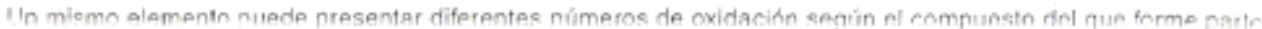
Veamos los números de oxidación más frecuentes que presentan algunos elementos signiticativos.

\begin{tabular}{|c|c|c|c|c|c|c|c|c|c|c|c|c|c|}
\hline Elemento & $H$ & \multicolumn{3}{|c|}{ Li, Na, K, Ab, Cs } & $\mathrm{Be}, \mathrm{Mg}, \mathrm{Ca}, \mathrm{Sr}, \mathrm{Ba}$ & $\mathrm{Fo}, \mathrm{Ca}, \mathrm{Ni}$ & \multicolumn{2}{|c|}{$\mathrm{Cu}$} & $\mathrm{Ag}$ & \multicolumn{2}{|c|}{ Au } & $\mathrm{Zn}, \mathrm{Cd}$ & $\mathrm{Hg}$ \\
\hline N." de oxidación & $-1,+1$ & \multicolumn{3}{|c|}{+1} & +2 & $+2,+3$ & \multicolumn{2}{|c|}{$+1,+2$} & +1 & \multicolumn{2}{|c|}{$+1,+3$} & +2 & $+1,+2$ \\
\hline Elemento & B & Al & C. Si & $\mathrm{Sn}, \mathrm{Pb}$ & $N$ & P, As, Sb & 0 & & $\mathrm{Se}, \mathrm{T}$ & & $F$ & $\mathrm{Cl}$, & $\mathrm{Br}, \mathrm{I}$ \\
\hline$N$." de oxidación & $-3,+3$ & +3 & $+4,-4$ & $+2,+4$ & $4-3,+1,+2,+3,+4$, & $5-3,+3,+5$ & -2 & -2 & $-2,-4$ & & -1 & $-1,+1$ & $3,+5,+7$ \\
\hline
\end{tabular}

Se puede determinar la fórmula de un compuesto, conocidos los números de oxidación. Y viceversa: si conocemos la formula de un compuesto, podemos determinar los números de oxidación.

Veamos unos ejemplos.

En ol tribxido de azutre, esfe elemento actía con nuimero de oxidacidn +6 . Determina su fórmula.

- El oxígeno actía siempre con número de oxidacion -2. Por tanto, para compensar el del azufre (en este caso +6) serán nocesarios 3 átomos de oxigeno

$+6-2$

$$
\mathrm{SO}_{3}-(+6)+3-(-2)=0
$$

-Un recurso útil para determinar el número de átomos necesarios consiste on eseribir sobro los simbolcs de los elementos sus números de oxidación y, a continuación, intercambiar. os y colocarlos como subindices, sin signo. Si procede. pueden simplificarse ambos valores. En este caso seria. recen en el sulfito do calcio, CaSO,

- El Ca y el $O$ actuan siempre con números de cxidación 1ijos: +2 y -2 , respectivamente. Tendremos, pues:

$$
\mathrm{Ca} \mathrm{SO}_{3}^{-2}
$$

- Llamamos x al número de ox dación del S. Según la regla bá. sica, la suma total de los números de oxidacion ha de ser O. Por tanto:

$$
+2+x+3 \cdot(-2)=0 \rightarrow x-44
$$

En el sulfito de calcio, el Ca actúa con nùmero de oxida. cón +2 , el $\mathrm{S}$ con +4 y el $\mathrm{O}$ con -2 .

$$
+2+4-2
$$

No se debe confundir el número de oxidación con la carga iónica.

- La carga iónica es la carga positiva o negativa que adquieren un átomo o grupo de átomos cuando pierden o ganan electrones. Se representa por n+ o n- en la parte superior derecha del ion.

- El numero de oxidación es una carga feórica, que representa la capacidad de combinación del elemento en un compuesto determinado. Se escribe sobre el simbolo del elemento de la forma $+n o-n$, durante el proceso de for. mulación y nomenclatura del compuesto pero no en la tórmula definitiva.

Dada la gran variedad y complejidad de compuestos inorgánicos existentes, nos vamos a limitar a analizar la formulación y la nomenclatura de los siguientes
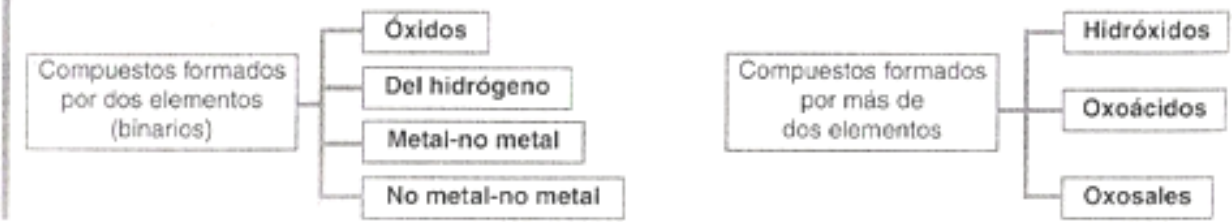
Como norma ganeral, cuanto más a la izcuierda está el elemento en el Sisterna Periódico, mayor es su carácter metálico. Si dos elementos están en un mismo grupo, tiene mayor carácter metálico el que sc encuentra má's abajo.

El orden on que se considera que disminuye el carácter metálico en los elementos no metálicos es el siguiente:

$$
\mathrm{B}, \mathrm{SI}, \mathrm{C}, \mathrm{Sb}, \mathrm{As}, \mathrm{P}, \mathrm{N}, \mathrm{H}, \mathrm{Te}, \mathrm{Se}, \mathrm{S}, \mathrm{I}, \mathrm{Br}, \mathrm{Cl}, \mathrm{O}, \mathrm{F}
$$

Veamos alqunas reolas qenerales para formular y nombrar comouestos binarios.

Formulación. Se escribe a la izquierda de la tórmula el simbolo del elemento con mayor carácler melélico.

El número de oxidación de un elemento se pone como subindios del otro, y se simplifica si es posible. Tendremos asi que la suma de los números de oxidación será 0 .
$\mathrm{Na}+1$
$\mathrm{Cl}-1 \rightarrow \mathrm{NaCl}$
$\mathrm{K}+1$
$\mathrm{S}_{-2} \Rightarrow \mathrm{K}_{2}$

Nomenclatura. En primer lugar se escribe el nombre del elemento con menor carácter metálico con la tẹrminación -uro, o, si se trala del oxígeno, con la palabra óxldo.

A continuación se escribe el nombre del elemento con mayor carácter melálico. Si sủlu ticno un número de oxidación, no se moclifica el nombre y se antepone la palabra de.

El subindice que debe llovar cada clemento se deduce de distinta forma según la nomenclatura utilizada, sistemática, de Stock y nombre clásico.

\begin{tabular}{|c|c|c|c|}
\hline & Nomenclatura sistemática & Nomenclatura de Slock & Nombre clásico \\
\hline & $\begin{array}{l}\text { So anicponen prefijos a los } \\
\text { nombres de los componentes } \\
\text { que hacen referencia a los sub- } \\
\text { índices (mono-, di-, tri-, tetra-, } \\
\text { penta-...). }\end{array}$ & $\begin{array}{l}\text { Cuando el elemento con mayor carácter } \\
\text { metálico tiene más de un número de oxi- } \\
\text { dación positivo, se indica aquél con el } \\
\text { que actúa mediante númoros romanos } \\
\text { entre paréntesis. }\end{array}$ & $\begin{array}{l}\text { La terminación -oso se utiliza } \\
\text { con el número de oxidaciún } \\
\text { más bajo, mientras que -ico } \\
\text { indica el más alto. }\end{array}$ \\
\hline $\mathrm{SnO}$ & Monóxido de estaño & Óxido de estaño (II) & Óxido estannoso \\
\hline $\mathrm{HgCl}_{2}$ & Diskerure de mercurio & Cloruro de mercurio (II) & Cloruro mercúrico \\
\hline
\end{tabular}

\section{Óxidos}

Las combinaciones del oxigeno con cualquilet oto olemento, excepto el flúor, se denominan óxidos.

Para formular los oxidos se escribe el oxígeno a la derecha on la fórmula. Éste actúa con número de oxidación -2 . El otro elemento se sitúa a la izquierda y actúa con número de oxidación positivo.

Para nombrar los óxidos se pueden utilizar las distintas nomenclaturas. Veamos unos ejemplos.

\begin{tabular}{|c|c|c|c|}
\hline & $\begin{array}{l}\text { Nomenclatura } \\
\text { sistemática }\end{array}$ & $\begin{array}{c}\text { Nomenclatura } \\
\text { de Stock }\end{array}$ & $\begin{array}{l}\text { Nombre } \\
\text { clásico }\end{array}$ \\
\hline $\mathrm{FeO}$ & Monóxido de hierro & Oxido de hierro (II) & Óxido ferroso \\
\hline $\mathrm{Fe}_{2} \mathrm{O}_{3}$ & Trióxido de dihierro & Óxido de hierro (III) & Oxido térrico \\
\hline $\mathrm{Al}_{2} \mathrm{O}_{3}$ & Trióxido de dialuminio & Óxido de aluminio & Oxido alumínico \\
\hline $\mathrm{SO}_{3}$ & Trióxido de azufre & Óxido de azulre (VI) & Óxido sulfürico \\
\hline $\mathrm{P}_{2} \mathrm{O}_{5}$ & Pentoxido de difosforo & Óxidọ de fósforo (V) & Óxido fosfórico \\
\hline
\end{tabular}

\section{Actividades}

- Utiliza la nomenclatura sistemática para formular y nombrar el óxido que se obtiene al unirse el axígeno con el elemento siguiente: a) cinc; b) plomo, con $n .{ }^{9}$ oxid. +2 ; c) azufre con $n .{ }^{9}$ oxid. +4 ; d) potasio; e) plata; f) oro con $n .{ }^{9}$ oxid. +3 .

- Formula los siguientes óxidos; a) óxido de cloro (l): b) trióxido de difósforo; c) dióxido de carbono; d) óxido de n/quel (III); e) dióxido de acuire.

- Utiliza la nomenclatura de Stock para nombrar los compuestos siguientes: aj $\mathrm{NiO}$; b) $\mathrm{Li}_{2} \mathrm{O}_{3}$ c) $\mathrm{B}_{2} \mathrm{O}_{3}$; d) $\mathrm{PbO}_{2}$; e) $\mathrm{CuO}$;) $\mathrm{HgO}$; g) $\mathrm{Au}_{2} \mathrm{O}$. 


\section{Combinaciones binarias del hidrógeno}

Clasificamos las combinaciones binarias del hidrógeno en hidrácidos, hidruros de otros no metales e hidruros metálicos.

\section{Hidrácidos}

Scor las combinaciones binarias del $\mathrm{H}$ con $\mathrm{F}, \mathrm{Cl}, \mathrm{Br}, \mathrm{I}, \mathrm{S}$, se y le.

Para formular estos compuestos el $\mathrm{H}$ se coloca a la izquierda del no metal. El $\mathrm{H}$ actúa con número de oxidación $+1 ; \mathrm{F}, \mathrm{Cl}, \mathrm{Br}$ y I actùan con número de oxidación -1, y S, Se y Te con -2.

Estas sustancias se denominan hidrácidos porque al disolverse en agua dan origen a ácidos. En este caso se nombran añadiendo la terminación -hidrico al nombre del halógeno o del calcogeno

\begin{tabular}{|c|c|c|c|}
\hline & $\begin{array}{l}\text { Nomenclatura } \\
\text { sistemática }\end{array}$ & $\begin{array}{l}\text { En disolución } \\
\text { acuosa }\end{array}$ & $\begin{array}{l}\text { Escribe la fórmula y nombra el compuesto formado por of hi. } \\
\text { drogeno y el futor. }\end{array}$ \\
\hline $\begin{array}{l}\text { HF } \\
\text { HC }\end{array}$ & $\begin{array}{l}\text { Fuoruro de hidrógeno } \\
\text { Cloruro de hidrógeno }\end{array}$ & $\begin{array}{l}\text { Acido fluorhidrico } \\
\text { Ácido clorhidrico }\end{array}$ & - E hidrógeno actúa con númeco de oxidación +1 y el thu \\
\hline $\mathrm{HBr}$ & Bromuro de hidrógeno & Acido bromhidrico & $\operatorname{con}-1$. \\
\hline HI & Yoduro de hidrógeno & Ácido yochidrico & $H F \Rightarrow(+1)+(-1)=0$ \\
\hline$H_{2} \mathrm{~S}$ & Suluro de dihidrógeno & Acido sulfhidrico & - Se nombra: fluoruro de hidrógeno. \\
\hline $\mathrm{H}_{2} \mathrm{So}$ & Seleniuro de dihidrogeno & Ácido selenhidrico & - En disolución acuosa, $\mathrm{HF}(a q)$, se nombra ácido fluorhictico. \\
\hline $\mathrm{H}_{2} \mathrm{~T}_{\mathrm{D}}$ & Telurure de dihidrogeno & Acido telurhidrico & \\
\hline
\end{tabular}

Acido telunhidrico

\begin{tabular}{|c|c|c|}
\hline & $\begin{array}{c}\text { Nomenclatura } \\
\text { sistemática }\end{array}$ & $\begin{array}{l}\text { Nombre } \\
\text { común }\end{array}$ \\
\hline $\mathrm{NH}_{4}$ & Trihidruro de niltrógeno & Amoniaco \\
\hline $\mathrm{PH}_{3}$ & Trihidrupo de fósfore & Fosfina \\
\hline $\mathrm{AsF}_{3}$ & Trihidruro de arsénico & Arsina \\
\hline $\mathrm{SbH}_{3}$ & Trihidruro de antimonio & Estibina \\
\hline $\mathrm{CH}_{1}$ & Metano & Metano \\
\hline \multirow[t]{2}{*}{$\mathrm{SH}$} & Tetrahidruro de silicio & Silano \\
\hline & $\begin{array}{c}\text { Nomenclatura } \\
\text { sistematica }\end{array}$ & $\begin{array}{c}\text { Nomenelatura } \\
\text { de Stock }\end{array}$ \\
\hline $\mathrm{NaH}$ & Hidruro de sodlo & Hidruro de sodio \\
\hline $\mathrm{CaH}_{2}$ & Dihidruro de calcio & Hidruro de calcio \\
\hline $\mathrm{PoH}_{4}$ & Tetrahidruro de plomo & Hidruro de plome \\
\hline
\end{tabular}

\section{Hidruros no metalicos}

El hidrogeno, con otros no metales, forma hidruros, que se conocen por sus nombres comunes. Consideraremos que el hidrógeno actúe con nümero de oxidación -1 .

Para formular estos compuestos se escribe el hidrógeno a la dere. cha, mientras que el elemento no metálico se coloca en primer lugar.

Observa la tabla de la izquierda. Podriamos considerar que el número de oxidación de N, P, As y Sb es +3 y el del C y Si, +4.

\section{Hidruros metálicos}

El hidrógeno actúa con los metales con número de oxidación -1 y forma hidruros metálicos.

Para formular los hidruros metálicos, el $\mathrm{H}$ se escribe a la derecha on la formula. En la mayoria de los casos el metal actúa con un único número de oxidación, por lo que no es necesario indicar éste.

\section{Actividades}

- Formula los siguientes compuestos: a) hidruro de esta$\tilde{n} \mathrm{O} ; \mathrm{b})$ dihidruro de calcio; c) ácido tolumidrico; d) hidruno de potasio; e) trihidrufo de aluminio; $f$ bromuro de hidrógeno.
- Nombra los aiguientes compuestos: a) $\mathrm{HBr}$; b) $\mathrm{SnH}_{4}$ : c) $\mathrm{RbH}$; d) $\mathrm{KH}$; e) $\mathrm{BaH}_{2}$; 7 BH, 


\section{Combinaciones metal-no metal}

Veamos las combinaciones de un metal con un no metal que no sea el hidrógeno ni el oxígeno. Son las llamadas sales binarias. Es el caso, por ejemplo, del cloruro de sodio, $\mathrm{NaCl}$.

Para formular estos compuestos el metal, que actúa con número de oxidación vositivo, sc colucé un la lómula dkanto y ol no motal, a continuación, puces to que actúa con número de oxidación negativo.

Escribe la tómula del compuesto formado por el cloro y el calcio.

- El cloro actúa con número de oxidación -1 y el cal cio con +2. Por lo tanto, son necesarios 2 átomos ce cloro por cada uno de calcio. I a fórmula es:

$$
\mathrm{Ca} \times \mathrm{Cl}
$$$$
\mathrm{CaCl}_{2} \rightarrow(+2)+2 \cdot(-1)=0
$$

Para nombrar estos compuestos se añade el sufjo -uro al nombre del no metal que interviene,

En el cuadro siguiènte se muestran algunos ejemplos de sales binarias.

\begin{tabular}{|c|c|c|c|}
\hline & $\begin{array}{l}\text { Nomenclatura } \\
\text { sistemática }\end{array}$ & $\begin{array}{l}\text { Nomenclatura } \\
\text { de Stock }\end{array}$ & $\begin{array}{l}\text { Nombre } \\
\text { clásico }\end{array}$ \\
\hline $\mathrm{NaCl}$ & Monocloruro de sodio & Cloruro de sodio & Cloruro de sodio \\
\hline $\mathrm{HgBr}$ & Monobromuro de mercurió & Bromuro de mercurio (I) & Bromuro mercurioso \\
\hline $\mathrm{Cul}_{2}$ & Diyoduro de cobre & Yoduro de cobre (II) & Yoduro cúprico \\
\hline $\mathrm{FeCl}_{3}$ & Tricloruro de hierro & Cloruro de hierro (III) & Cloruro térrico \\
\hline NiSe & Monoseleniuro de níquel & Seleniuro de niquel (II) & Seleriuro niqueloso \\
\hline $\mathrm{Ni}_{2} \mathrm{~S}_{3}$ & Trisulfuro de diniquel & Sulfuro de níquel (III) & Sulfuro niquélico \\
\hline $\mathrm{SnF}_{4}$ & Tetrafluoruro de estaño & Fluoruro de estano (IV) & Fluoruro estánnico \\
\hline $\mathrm{Hg}_{3} \mathrm{As}_{2}$ & Diarseniuro de trimernurio & Arsen uro de mercurio (II) & Arseniuro mercúrico \\
\hline
\end{tabular}

Nombra la siguiente sal: $\mathrm{CU}_{2} \mathrm{~S}$. Utiliza las nomenclaturas de Stock y sistemática.

- El no metal ess ul S. Por tanto, será un sulfuro.

El Cu actúa con número de oxidación +1 , el menor de los dos que posee.

En nomenclatura de Stock el compuesto se nombra: monosulfuro de dicobre (i) O, simplemente, sulfuro de dicobre (I)

- En nomenclatura sistemática se nombra: monosiulfuro de dicobre.

\section{Actividades}

- Formula y nombra los compuestos formados por los elementos siguientes. Utiliza la nomenclatura de Stock
a) Cloro y níquel (n. "oxid. +2)
b) Nitrógeno y calcio
c) Azutre y sodio
d) Bromo y plata

- Nombra los compuestos siguientes. Utiliza la nomenclatura sistemática.
a) $\mathrm{K}$
b) $\mathrm{MgCl}$
c) $\mathrm{CdF}_{2}$
d) $\mathrm{HgS}$
e) $\mathrm{Pbl}_{4}$
f) $\mathrm{CoBr}_{3}$
g) $\mathrm{FeSe}$ 


\section{Combinaciones no metal-no metal}

Veamos a continuación los compuestos que se forman entre dos no metales que no son óxidos ni combinaciones del hidrógeno, ya vistos.

Para formular estos compuestos, se escribe en primer lugar el elemento que tenga mayor carácter metálico, que actuará con número de oxidación posilivo.

Estas combinaciones se nombran como compuestos del no metal que actúa con número de oxidación negativo. Observa unos ejempios.

\begin{tabular}{|c|c|c|}
\hline & $\begin{array}{l}\text { Nomenclatura } \\
\text { sistemática }\end{array}$ & $\begin{array}{c}\text { Nomenclatura } \\
\text { de Stock }\end{array}$ \\
\hline $\mathrm{IBr}$ & Monobromuro de yodo & Bromuro de yodo (I) \\
\hline $\mathrm{P}_{2} \mathrm{Te}_{3}$ & Tritelururo de difósforo & Telururo de fósforo (Mi) \\
\hline $\mathrm{S}_{\Theta C l}$ & Dicloruro de selenio & Cloruro de selenio (II) \\
\hline
\end{tabular}

\section{Hidróxidos}

Los hidróxidos están formados por un metal, el oxigeno y el hidrógeno. Estos dos últimos aparecen agrupados formando un ion, el $\mathrm{OH}^{-}$, que recibe el nombre de ion hidróxido y que actúa como si fuera un elemento con número de oxidación -1.

Para formular los hidróxidos se escribe en primer lugar el metal y, a continuación, el ion hidróxido.

Si el metal tiené número de oxidación +1 , no es preciso poner ningún subíndice; pero si su número de oxidación es otro, se coloca el grupo $\mathrm{OH}^{-}$entre parćntesis y se le asigna el subindice correspondiente.

Escribe las fórmulas del hidróxido de potesio y del hidróxido de magnesio

- Escribimos sobre los símbolos de los metales y del grupo $\mathrm{OH}$ sus respectivos números de oxidación. En el caso del hidróxido de potasio no es necesario colocar ningún subíndice, pero en el del hidróxido de magnesio hay que escribir el grupo $\mathrm{OH}$ entre paréntesis y asignarle el subíndice 2 .

$$
\begin{aligned}
& \text { K. } \mathrm{OH}^{-1} \\
& (+1)+(-1)=0
\end{aligned}
$$

Pârả nombrar los hidróxidos sé ántepone la expre sión hidróxido de... al nombre del metal.

Habitualmente suele emplearse la nomenclatura do Stock.

Observa cómo se utilizan las nomenclaturas Stock y sistemática en los ejemplos de la tabla adjunta.

$$
\stackrel{+2}{\mathrm{Mg}_{2}} \underset{(+2)+2 \cdot(-1)=0}{\stackrel{\mathrm{O}}{\longrightarrow} \underset{\mathrm{Mg}}{\longrightarrow}(\mathrm{OH})}
$$

\begin{tabular}{lll} 
& \multicolumn{1}{c}{$\begin{array}{c}\text { Nomenclatura } \\
\text { de Stock }\end{array}$} & \multicolumn{1}{c}{$\begin{array}{c}\text { Nomenclatura } \\
\text { sistematica }\end{array}$} \\
\hline $\mathrm{NaOH}$ & Hidróxido de sodio & Hidróxido de sodio \\
$\mathrm{Al}(\mathrm{OH})_{3}$ & Hidróxido de aluninio & Trihhidróxido de alurnitio \\
$\mathrm{Fe}(\mathrm{OH})_{2}$ & Hidróxido de hierro (II) & Dihidróxido de hierro \\
$\mathrm{Fe}(\mathrm{OH})_{3}$ & Hidróxido de hierro (III) & Trihidróxido de hierro
\end{tabular}

\section{Actividades}

- Nombra lós siguientes compuesstos: a) $\mathrm{P}_{5} \mathrm{Cl}_{3}$; b) SbN c) $\mathrm{CuOH}$; d) $\mathrm{Sr}(\mathrm{OH})_{2}$; e) $\mathrm{HgOH}$; f) $\mathrm{Ni}(\mathrm{OH})_{3}$;g) $\mathrm{Pb}(\mathrm{OH})_{4}$ h) $\mathrm{Hg}(\mathrm{OH})$.
- Formula y nombra los hidróxidos de: a) plata; b) cobalto $\left(n .^{\circ}\right.$ oxid. +2$)$; c) cobre (n. ${ }^{\circ}$ oxid. +2$)$; d) níque (n. oxid. +3 ); e) calcio, Utiliza la nomenclatura de Stock. 
En algunos casos, el elemento central de un oxoeicido puede ser un metal. Es el caso del cromo y el manganeso, que dan origen a diversos ácidos, como, por ejemplo, $\mathrm{H} \mathrm{CrO}$ ácldo crómlco $\mathrm{H}$ Cr.O ácido dicromico, $\mathrm{HMnO}_{4}$ acido permangénico

\section{Oxoácidos}

Los oxoácidos son compuestos ternarios, es decir, formados por tres ele mentos: el hidroggeno, un elemento no metálico (con número de oxidación positivo) y el oxígeno. Se trata de uno de los grupos de sustancias más importantes dentro de la química.

La fümula general de lus uxuacidos es $\mathrm{H}_{\theta} \mathrm{X}_{b} \mathrm{O}_{c}$. El hidiogeno actua con nimero de oxidación +1; el elemento no metálico, con un número de oxidación positivo; y el oxigeno, con número de oxidación -2

Habitualmente $b=1$. Veamos cómo formular estos ácidos

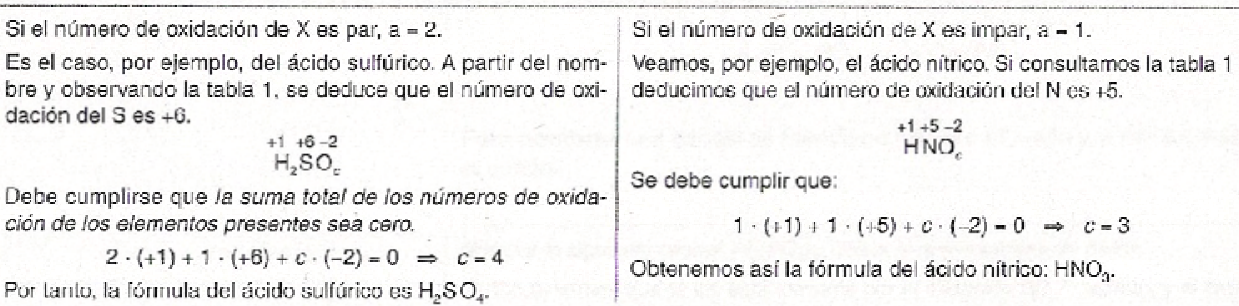

Para nombrar los oxoácidos, aunque existe un nombre sistemático para cada uno de ellos, suele utilizarse el nombre clásico. La regla que se utiliza para nombrar estos compuestos se basa en la cantidad de números de oxidación que tiene el elemento no metálico y es la siguiente:

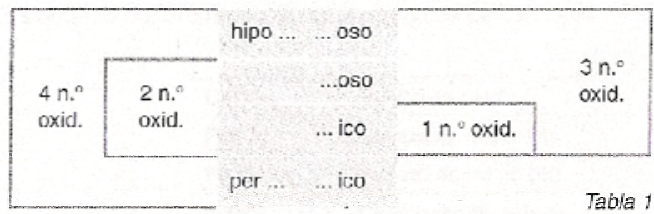

Observa cómo el cloro forma cuatro oxoácidos, con los cuatro números de oxidación positivos que tiene: $+1,+3,+5 y+7$.

De igual forma, el azufre forma tres oxoácidos, con los números de oxidación: $+2,+4$ y 16 .

En el único ácido que forma, el carbono actúa con número de oxidación +4

Aquí tienes algunos de los oxoácidos más importantes.
\begin{tabular}{clll} 
Oxoácido & Nombre clásico & Oxoácido & \multicolumn{1}{c}{ Nombre clásico } \\
\hline $\mathrm{HClO}$ & Ácido hipocloroso & $\mathrm{H}_{2} \mathrm{SO}_{2}$ & Ácido hiposulturoso \\
$\mathrm{HClO}_{2}$ & Ácido cloroso & $\mathrm{H}_{2} \mathrm{SO}_{3}$ & Ácido sulfuroso \\
$\mathrm{HClO}_{3}$ & Ácido clórico & $\mathrm{H}_{2} \mathrm{SO}_{4}$ & Ácido sulfúrico \\
$\mathrm{HClO}_{4}$ & Ácido perclórico & $\mathrm{HNO}_{2}$ & Ácido nitroso \\
$\mathrm{HBrO}_{3}$ & Ácido brómico & $\mathrm{HNO}_{3}$ & Ácido nítrico \\
$\mathrm{H}_{2} \mathrm{CO}_{3}$ & Ácido carbónico & $\mathrm{H}_{3} \mathrm{PO}_{4}$ & Ácido fosfórico
\end{tabular}

\section{Actividades}

- Deduce el número de oxidación del elemento no metálico en los ácidos siguientes: a) $\mathrm{HNO}_{2}$; b) $\mathrm{H}_{2} \mathrm{CO}_{3}$; c) $\mathrm{H}_{2} \mathrm{SO}_{3}$

- Formula los siguientes ácidos: a) ácido bromoso; b) ácido peryódico; c) ácido selenioso; d) ácido hipobromoso.

- Nombra los siguientes ácidos: a) $\mathrm{HBrO}_{4}$; b) $\mathrm{H}_{2} \mathrm{TeO}_{4} ;$ c) $\mathrm{HIO}_{3}$. 


\section{Oxosales}

Antes de proceder al estudio de lás oxosales debemos profundizar en la nomenclatura y formulaclón do los iones.

\section{lones}

Los lones son átomos o grupos de átomos que han perdido o ganado electrones, por lo que tienen carga positiva o negativa, respectivamente. Los iones de carga positiva se llaman cationes y los de carga negativa, aniones.

Veamos estos iones, según sean monoatómicos o poliatómicos.

\section{Cationes monoatómicos}

Para formular estos cationes se escribe el símbolo del elemento y un superíndice que nos indica la carga. Esta carga coincidirá con uno de los estados de oxidación.

Así tenemos, por ejemplo: $\mathrm{Fe}^{2+}, \mathrm{Fe}^{3+}, \mathrm{Na}^{+}$.

Para nombrar los cationes utilizaremos la nomençatura de Stock. Anteponemos la palabra ion o catión al nombre del elemento. Si tiene varios números de oxidación, se indiça con el que actúa en números romanos, entre paréntesis.

Los cationes monoatómicos los forman los metales. Por ejemplo: $\mathrm{Fe}^{2+}$, ion hierro (II): $\mathrm{Fe}^{3+}$, ion hierrn (III); $\mathrm{Na}^{+}$, ion sodio.

\section{Aniones monoatómicos}

Para formular los aniones monoatómicos se escribe el símbolo del elemento y un superíndice que nos indica la carga. Esta carga coincide con el estado de oxidación negativo del elemento.

Por ejemplo: $\mathrm{S}^{2-}, \mathrm{Cl}^{-}, \mathrm{N}^{3}$.

Para nombrar estos aniones se utiliza la terminación -uro. No hace falta indicar la carga, ya que ésta coincide con el número de oxidación negativo del elemento.

Los aniones monoatómicos los forman los no metales. Así, tenemos: $\mathrm{S}^{\text {?- }}$, ion sulfuro: $\mathrm{Cl}^{-}$, ion cloruro; $\mathrm{N}^{3-}$, ion nitruro. $\mathrm{El}$ nombre del anión del oxígeno, $\mathrm{O}^{2-}$, $\Theta$ s una excepción; sc nombra ion úxido.

\section{Cationes pollatómicos}

Existen dos muy importantes, el $\mathrm{NH}_{4}{ }^{+}$, ion amonio y el $\mathrm{H}_{3} \mathrm{O}^{*}$, ion hidronio.

\section{Aniones poliatómicos}

Ya conocemos un caso, el ion hidróxido $\mathrm{OH}$. Veamos a cuntinuación los aniones que provienen de los oxoácidos, los oxoaniones.

Para formular los oxoaniones se parte de la fórmula del ácido, se quitan los hidrógenos y se ponen tantas cargas negativas como hidrógenos hemos quitado.

Asi, por ejemplo, ténemos: $\mathrm{H}_{2} \mathrm{SO}_{4} \Rightarrow \mathrm{SO}_{4}^{2-}, \mathrm{HNO}_{2} \rightarrow \mathrm{NO}_{\text {, }}$

Para nombrar los oxoaniones sé cambian las terminaciones -oso e -ico de los ácidos por -ito y -ato, respectivamente.

Tendremos, por tanto, que el $\mathrm{SO}_{4}^{z-}$ es el ion sulfato y el $\mathrm{NO}_{2}^{-}$ion nitrito

En el sigulente cuadro se presentan algunos oxoaniones comunes.

\begin{tabular}{llllll} 
Ion Nombre clásico & Ion & Nombre clásico & Ion & Nombre clásico \\
\hline $\mathrm{ClO}^{-}$ & ion hipoclorito & $\mathrm{BrO}_{3}^{-}$ & ion bromato & $\mathrm{NO}_{2}^{-}$ & ion nitrito \\
$\mathrm{ClO}_{2}^{-}$ion clorito & $\mathrm{IO}_{3}^{-}$ & ion yodato & $\mathrm{NO}_{3}$ & ion nitrato \\
$\mathrm{ClO}_{3}^{-}$ & inn clorato & $\mathrm{SO}_{3}{ }^{2-}$ & ion sulfito & $\mathrm{CO}_{3}{ }^{2-}$ & ion carbonato \\
$\mathrm{ClO}_{4}^{-}$ & inn perclorato & $\mathrm{SO}_{4}{ }^{2-}$ & ion sulfato & $\mathrm{PO}_{4}{ }^{3-}$ & ion fosfato
\end{tabular}

\section{Actividades}

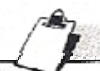

- Formula y nombra los cationes de: a) oro; b) estaño; c) estroncio; d) magnesio; e) cobre.

- Formula y nombra lós aniones de los oxoácidos del yodo 
Las oxosales son los compuestos que se obtienen por sustitución del hidrógeno o los hidrógenos de los oxoácidos por un elemento metálico, por lo que están formadas por un catión de un metal y un oxoanión.

Para formular una oxosal, se escribe, en primer lugar, el catión y, a continuación, el oxoanión. Los subíndices que corresponden a cada uno pueden obtenerse intercambiando las respectivas cargas. En caso de que sea posible, lus subindices se simplifican.

Escribe la fórmula de la sal que forma el ion fosfato con el jon calcio.

- Prucedernos de la forma habitual. Tenóremos:

$$
\stackrel{+2}{\mathrm{Ca}} \underset{\mathrm{PO}}{-3} \longrightarrow \mathrm{Ca}_{3}\left(\mathrm{PO}_{4}\right)_{2}
$$

Para nombrar una oxosal se menciona. primero el anión y, a continuación, el catión.

Nombra la siguiente oxosal: $\mathrm{Al}_{2}\left(\mathrm{SO}_{4}\right)_{3}$. Utiliza la nomenclatura de Stock.

- Observamos que la sal está formada por el oxoanión $\mathrm{SO}_{4}{ }^{2-}$, sulfato, y el catión $\mathrm{Al}^{3}$, ion aluminio.

$$
\left(\mathrm{Al}^{3}\right)_{2}\left(\mathrm{SO}_{4}^{2-}\right)_{3}
$$

- En la nomenclatura de Stock nombramos primero el oxoanión y en segundo lugar el elemento metálico: sulf́ato de aluminio.

El siguiente cuadro muestra otros ejemplos de oxosales.

\begin{tabular}{ll}
\hline $\mathrm{CaSO}_{3}$ & Sulfito de calcio \\
\hline $\mathrm{FeSO}_{4}$ & Sulfato de hierro (II) \\
\hline $\mathrm{Fe}_{2}\left(\mathrm{SO}_{4}\right)_{3}$ & Sulfato de hierro (III) \\
\hline $\mathrm{K}_{2} \mathrm{CO}_{3}$ & Carbonato de potasio \\
\hline $\mathrm{CuNO}_{3}$ & Nitrato de cobre (I) \\
\hline $\mathrm{Cu}\left(\mathrm{NO}_{3}\right)_{2}$ & Nitrato de cobre (II) \\
\hline $\mathrm{AgNO}$ & Nitrito de plata \\
\hline $\mathrm{Ni}(\mathrm{ClO})_{3}$ & Hipoclorito de n'quel (III) \\
\hline $\mathrm{HglO}$ & Yodato de mercurio (I) \\
\hline
\end{tabular}

\section{Actividades}

- Formula las sales que se obtienen al unírse los aniones y cationes siguientes: a) sulfato y los jones del hierro; b) nitrato e ion plata; c) hipoclorito e ion calcio; d) perbromato y los iones del cobre.

- Nombra las sales siguientes: a) $\mathrm{ZnCO}_{3}$; b) $\mathrm{Ag}_{3} \mathrm{PO}_{4}$; c) $\mathrm{Ba}\left(\mathrm{NO}_{3}\right)_{2}$; d) $\mathrm{Al}\left(\mathrm{ClO}_{3}\right)_{3}$; e) $\mathrm{NaClO}$; f) $\mathrm{HglO}_{4}$.

- Formula las sales siguientes: a) clorato de plata; b) hipoclorito de estaño (IV); c) perclorato de calcio; d) nitrito de potasio; e) carbonato de magnesio; f) sulfito de cadmio.

- Nombra las siguientes sales y comprueba que la suma de los números de oxidación da $\left.0: a) K_{2} \mathrm{CO}_{2} ; b\right) \mathrm{Ca}_{5}\left(\mathrm{PO}_{4}\right)_{2}$. 


\section{ANEXO II: Bullejos, J., Carmona, A., Hierrezuelo, J., Molina, E., Montero, A., Mozas, T., Ruiz, G., Sampedro, C., del Valle, V. (2008). Física y Química 4º ESO. Granada: Elzevir}

Salvo en el caso de los iones, todas las fórmulas se escriben sin carga, lo que corresponde al estado neutro de las sustancias que representan. Por ello, el número de electrones que puedan perder alguno de los elementos debe ser igual al de los electrones ganados por otros, como puedes ver en los siguientes ejemplos:

Fjemplo 1: ¿Qué fórmula corresponde a una sustancia formada por los elementos cloro y sodio?

Puess co que cada átomo de clom gana 1 elertrón mientras que cado tomo de sodio pierde un electrón, deberá haber el mismo numero de átomos de sodio que de cloro. Por lo lanlo, la fórmula debería ser: $\mathrm{Na}_{\mathrm{n}} \mathrm{Cl}_{\mathrm{n}}$. Puesto que se procura poner la fórmula más simple, en lugar de escribir la antetior, escribiremos: $\mathrm{Na}_{1} \mathrm{Cl}_{1}$. Se ha adoptado el convenio de que cuando el subindice sea un 1 no se escriba. Por lo tanto, la fómula será: $\mathrm{NaCl}$.

Ejemplo 2: ¿Qué fórmula corresponde a una sustancia formada por los elementos cloro y calcio?

Puesto que cada átomo de cloro gana 1 electrón mientras que cada átomo de calcio pierde 2 electrones, deberá haber el doble número de átomos de cloro que de calcio. Por lo tanto, la fórmula debería ser: $\mathrm{Ca}_{n} \mathrm{Cl}_{2 n}$. Por las razones expuestas en el ejemplo anterior, la fórmula serń: $\mathrm{CaCl}_{2}$.
Reglilla para formulación «automática»

Se trata de intercambiar las valencias. Así, si el hierro actủa con valencia 3 mientras que el oxigeno lo hace con valencia 2 , la fórmula del óxido de hierro (III) será $\mathrm{Fe}_{2} \mathrm{O}_{3}$

El número entre parentesis se refiere a la valencia con la que actúa el hipro Fs necesario ponerlo en el caso de que ese elemento pueda actuar con valenclas diferentes.

En caso de que sea posible, se simplificarian lexi númerns: que apkarecen como subíndices. Así, óxido de calcio: $\mathrm{Ca}_{2} \mathrm{O}_{2}$, se debe escribir, CaO.

En ocasiones no se puede simplificar una fómula porque representa a una molécula con una composición atómica determinada. Asi, el peróxldo de hidrógeno se escribe $\mathrm{H}_{2} \mathrm{O}_{2}$.

\subsection{Formulación de compuestos binarios}

\section{Hidruros}

Son las combinaciones del hidrógeno con los demás elementos. Se nombran, según la nomenclatura sistemática de la IUPAC, al contrario de como se escriben, terminando siempre el nombre del primer elemento con el sufijo -uro.

De todas formas algunos conservan su nombre tradicional.

\begin{tabular}{|c|c|c|c|c|c|c|c|}
\hline \multicolumn{8}{|c|}{$\begin{array}{l}\text { Valencias que utilizan los elementos } \\
\text { representativos con el hidrógeno }\end{array}$} \\
\hline Grupo & 1 & 2 & 13 & 14 & 15 & 16 & 17 \\
\hline Valencia & 1 & 2 & 3 & 4 & 3 & 2 & 1 \\
\hline
\end{tabular}

\begin{tabular}{|c|c|c|}
\hline $\begin{array}{l}\text { Fórmula } \\
\mathrm{H}^{2}\end{array}$ & $\begin{array}{l}\text { N. sistemática } \\
\text { fluórurò de hideúgéne }\end{array}$ & $\begin{array}{l}\text { N. tradicional } \\
\text { úcido fluorhídricu (en agua) }\end{array}$ \\
\hline HOI & cloruro de hidrógeno & ácido clorhídrico (" ") \\
\hline $\mathrm{HBr}$ & bromuro de hidrógeno & ácido bromhídrico (" ") \\
\hline $\mathrm{HI}$ & yoduro de hidrógeno & ácido yodhídrico ("“ ") \\
\hline $\mathrm{H}_{2} \mathrm{~S}$ & sulfuro de hieirúgene & ácido sulfhídrico (" ") \\
\hline $\mathrm{H}_{2} \mathrm{Se}$ & seleniuro de hidrógeno & ácido selenhídrico (" ") \\
\hline $\mathrm{NH}_{3}$ & hidruro de nitrógeno & amoníaco \\
\hline $\mathrm{PH}_{3}$ & hidruro de fósforo & fosfina \\
\hline $\mathrm{AsH}_{3}$ & hidruro de arsénico & arsina \\
\hline $\mathrm{SbH}_{3}$ & hidruro de antimonio & estibina \\
\hline $\mathrm{SiH}_{4}$ & hidruro de silicio & silano \\
\hline $\mathrm{BH}_{3}$ & hidruro de boro & borano \\
\hline
\end{tabular}

\begin{tabular}{|c|}
\hline $\begin{array}{l}\text { ¿Qué símbolo se escribe a la } \\
\text { izquierda y cuál a la derecha? }\end{array}$ \\
\hline $\begin{array}{l}\text { Una regla sencilla para recor- } \\
\text { dar es que se escriben al revés de } \\
\text { como se nombran. Por ejemplo, el } \\
\text { bromuro dr troditu see texcithe: Nabr. }\end{array}$ \\
\hline $\begin{array}{l}\text { El hidrógeno se escribe a la } \\
\text { derecha excepto cuando se combina } \\
\text { con elementos de los grupos } 16 \text { y } 17 \text {. } \\
\text { Ejemplos: } \mathrm{KH}, \mathrm{CaH}_{2}, \mathrm{AlH}_{3}, \mathrm{H}_{2} \mathrm{~S}, \mathrm{HBr} \text {. }\end{array}$ \\
\hline $\begin{array}{l}\text { El oxígeno siempre se escribe } \\
\text { a la derecha, excepto cuando se com- } \\
\text { bina con el flúor. Ejemplo: CaO. }\end{array}$ \\
\hline $\begin{array}{l}\text { Si hay tres elementos, el hidró- } \\
\text { geno o el metal se escriben a la iz- } \\
\text { quierda, el no metal en el centro y el } \\
\text { oxígeno a la derecha. Ejemplos: } \\
\mathrm{HNO}_{3} \mathrm{CaSO}_{4} \text {. }\end{array}$ \\
\hline
\end{tabular}

2. UNIÓN ENTRE ÁTOMOS Y PROPIEDADES DE LAS SUSTANCIAS 

Bruño

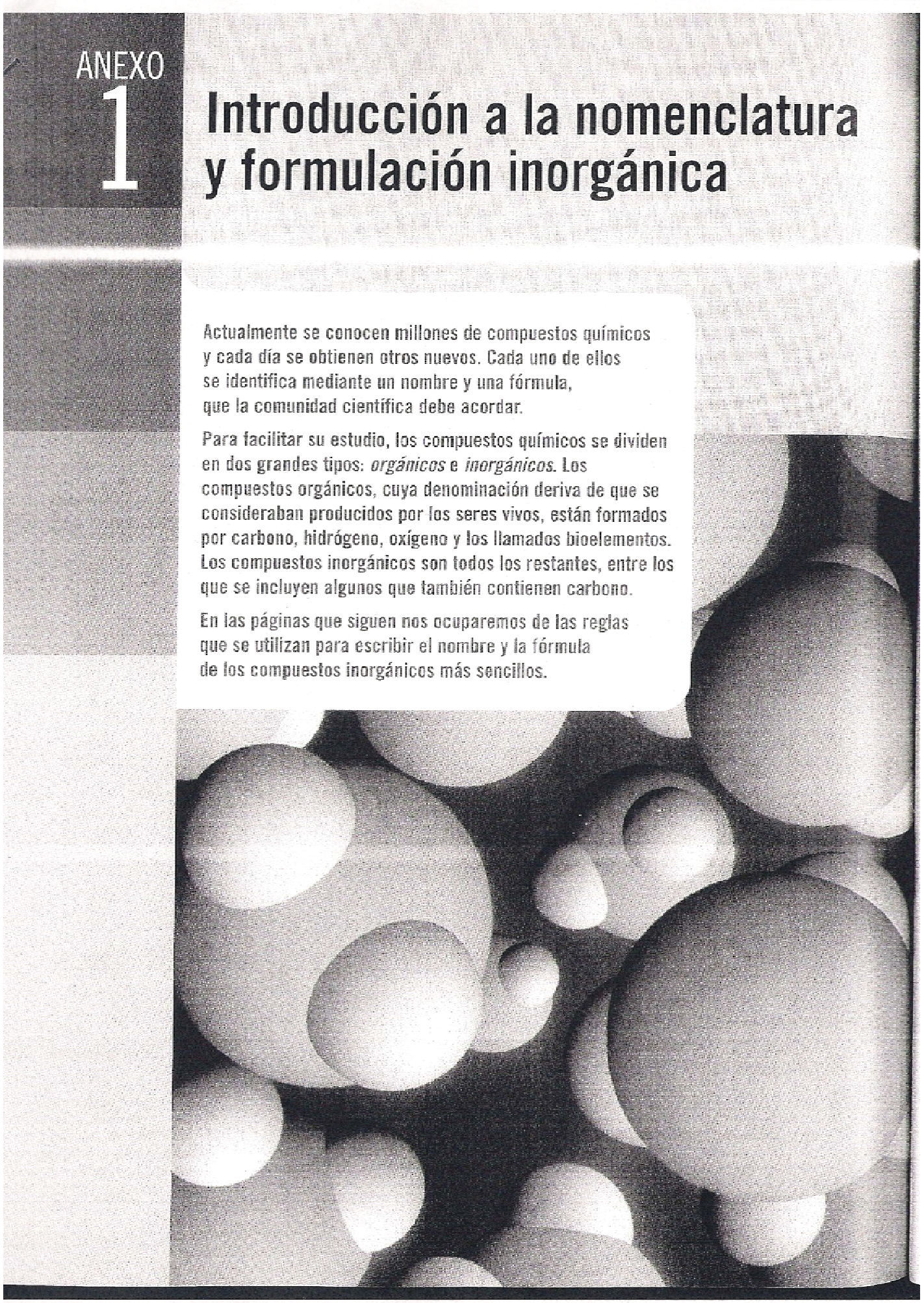




\section{Formulación y nomenclatura}

Como sabemos, cada compuesto quimico se representa mediante una fórmula, que expresa los elementos quimicos que lo forman y la proporción en la que se encuentran los átomos de esos elementos.

Cuando se conocian pocos compuestos químicos, estos recibian un nombre que hacía referencia a sus propiedades 0 a las circunstancias en que fueron descubiertos. Por ejemplo el compuesto de fórmula $\mathrm{NaOH}$ se denominaba sosa cáustica Con el paso de tiempo, el gran número de compuestos conocidos hizo necesario recurrir a ciertas reglas para asignarles nombres

La nomenclatura es un conjunto de reglas que permite asignar un nombre especifico y descriptivo a un compuesto dado por su fórmula. La formulación consiste en obtener la fórmula de un compuesto a partir de su nombre.

Existen tres sistemas de nomenclatura aceptados por la IUPAC (Unión Internacional de Quimica Pura y Aplicada): la nomenclatura sistemática, recomendada por la IUPAC para facilitar la comunicación científica, la nomenclatura de Stock y la nomenclatura tradicional, más antiqua y en desuso para algunos tipos de compuestos.

\section{Clasificación de los compuestos inorgánicos sencillos}

Los compuestos inorgánicos que estudiaremos se clasifican en binarios, que están formados por dos elementos distintos, y ternarios, que contienen tres elementos diferentes. Estos dos grupos se subdividen a su vez, dando lugar a seis tipos de compuestos, como se muestra en la siguiente tabla

\begin{tabular}{|c|c|c|}
\hline \multirow{3}{*}{ Binarios } & Óxidos & Formados por owigeno y otro elemento. \\
\hline & Hidruros & Formados por hidrógeno y otro elemento. \\
\hline & Sales binarias & $\begin{array}{l}\text { Formadas por dos elementos distintos del hidrógeno y el oxigeno: } \\
\text { un metal y un no metal. }\end{array}$ \\
\hline \multirow{3}{*}{ Temarios } & Oxoácidos & Formados por oxigeno, hidrógeno y un tercer elemento, que suele sér no metálica. \\
\hline & Hidróxidos & Formados por un metal y oxigeno e hidrógeno, formando grupos $\mathrm{OH}$. \\
\hline & $\begin{array}{l}\text { Sales ternarias } \\
\text { u oxisales }\end{array}$ & $\begin{array}{l}\text { Formadas por oxigeno y otros dos elementos, generalmente un metal } \\
\text { y un no metal. }\end{array}$ \\
\hline
\end{tabular}

Clasificación de los compuestos inorgánicos sencillos.

\section{ACTIVIDADES}

7e Clasifica estos compuestos inorgánicos de acuerdo con su fórmula:

a) Dihidruro de cobre, $\mathrm{CuH}_{2}$

b) Ácido sulfúrico, $\mathrm{H}_{2} \mathrm{SO}_{4}$.

c) Cloruro de sodio, $\mathrm{NaCl}$.

d) Trióxido de dihierro, $\mathrm{Fe}_{2} \mathrm{O}_{3}$.

e) Fosfato de aluminio, $\mathrm{AlPO}_{4}$

f) Monóxido de carbono, $\mathrm{CO}$.

g) Trihidroxido de aluminio, $\mathrm{Al}(\mathrm{OH})_{3}$.

Una organización internaci

La IUPAC surgió en 1919, tras rios intentos de los químicos epoca por unificar el lenguaje mico. Para entonces, la Quín tuabe poro mitis de un ight desarroilo como ciencia, pero enorme la cantidad de desco hizo necesario un acuerdo in nacional, con el fin de estable referencias y terminclogia ar piadas para el trahajo cuímic: 


\author{
Valencias \\ La valencia do un elemento quimi- \\ co es un número que cuantitica \\ sus posbilidades de combinación \\ con otros elementos. Aunque es \\ mero de oxidación, en la práctica \\ mero de oxidación, en la practica \\ mento es el del número de oxida- \\ ción considerado siempre positivo.
}

\section{¿Derecha o izquierda?}

Scgún las normas de la IUPAC. en la formula dohemos escribit a la izquierda los elementos o grupos con números de oxidatión o carga positivos, y o ld dercrhis. los nue puseell numeros de oxidación o carcas negativos.
Es un hecho experimental comprobado que los elementos quimicos pueden combinarse en varias proporciones para dar lugar a distintos compuestos. Por ejerruplo, ol azufre y el oxígcno forman dos compuestos con propiedades diferentes el dióxido de azufre $\left(\mathrm{SO}\right.$, ) y cl trińxido de azufre $\left(\mathrm{SO}_{3}\right)$.

Para determinar las distintas posibilidades de combinación de un elementu dado, se define el concepto de número de oxidación

[l número de oxidución de un elemento en un compuesto químico $\subseteq$ un número entero que pepresenta la carga nue le corresponde, considerando que fl compuesto es iónico, dun cuando presente otro tipo de enlace.

A partir de las fórmulas de los conpuestus, se calculan los números de oxidación que utilizan los elementos al combinarse. Es los pucden ser positivos o negativos, dependiendo de si el elcmento tiene tendencia a ceder o a tomar electrones, respectivamente, al unirse a otms elementos.

En un compuesto neutro, la suma ta las cargas aportadas pur cada elemento a través de sus numeros de oxidación debe ser cero; si se trata de un ion, la suma de las cargas us igual a la carga del ion.

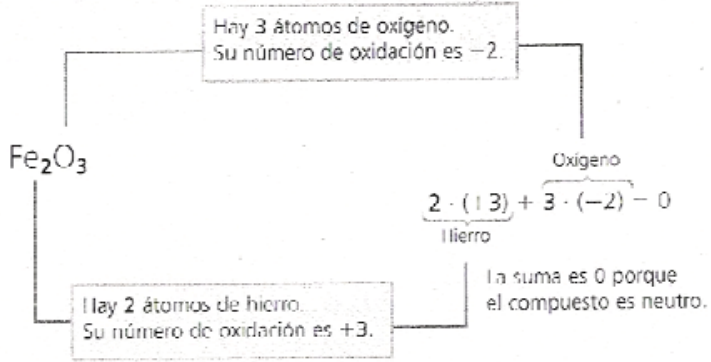

Fn esta tabla se muestran los números de cxidación correspondientes a los elementos más habituales:

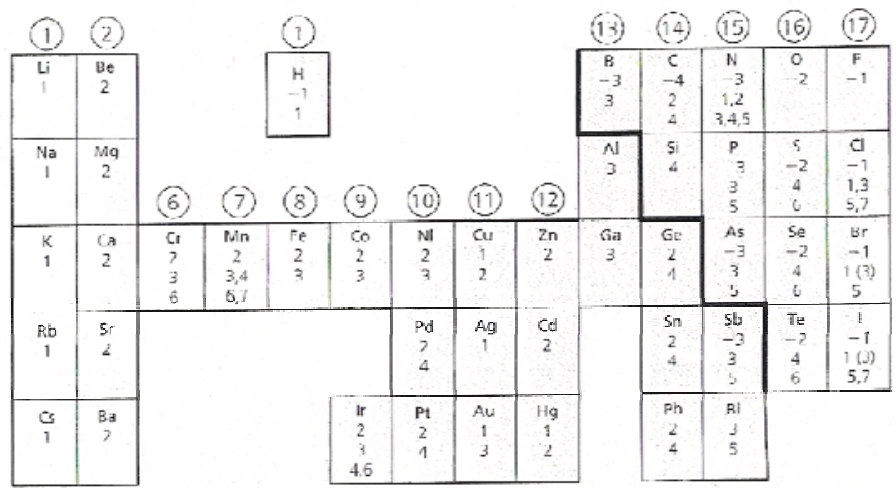

Los números de oxidaciún indicados entre parentesis no se presentan en compursios sitables y solo se consideran a efectos de nomenrlatura. 


\section{Nomenclatura y formulación de compuestos binarios}

\section{D́xidos}

Los óxidos son compuestos binarios en los cuales uno de los elementos es el oxígeno, que actúa con número de oxidación -2 .

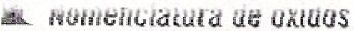

Para nombrar los óxidos, se utilizan la nomenclatura sistemática y la de Stock.

Segứn là nomenclatura sistemática, se nombran escribiendo la palabra óxido precedida de un prefijo (di-, tri-, tetra-, etc.) que indica el número de átomos de oxígeno que hay en el compuesto, seguido de la preposición de y del nombre del elemento que acompaña al oxígeno, precedido también del correspondiente prefijo numeral:

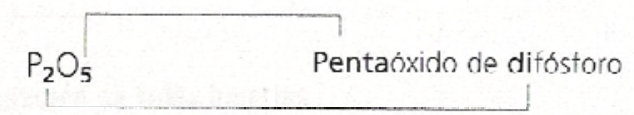

Según la nomenclatura de Stock, la palabra óxido và seguida de la preposición de y del nombre del elemento que se combina con el oxígeno; entre paréntesis, sin signo y en números romanos, se indica el número de oxidación del elemento que acompaña al oxígeno, en el caso de que tenga varios:

$$
\mathrm{P}_{2} \mathrm{O}_{5} \rightarrow \text { Oxido de fósforo }(\mathrm{V})
$$

Formulación de óxidos

A partir del nombre sistemático es fácil escribir la fórmula de un óxido, teniendo en cuenta que los prefijos numerales nos indican el número de átomos de cada elemento que hay en el compuesto. En la fórmulà, el oxígeno debe aparecer escrito a la derecha.

\section{Trióxido de dihierro}

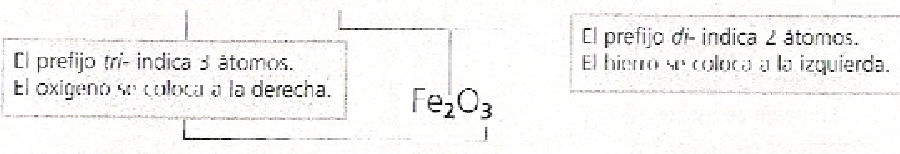

Si el nombre viene dado por la nomenclatura de Stock, y considerando que en los óxidos el número de oxidación del oxígeno es -2 , se escribe el elemento que acompaña al oxígeno con subindice 2 , y el oxigeno a la derecha, con un subíndice que viene dado por el número de oxidación del otro elemento. Si es posible, se simplificarán los subíndices obtenidos, de modo que queden en su expresión más sencilla.

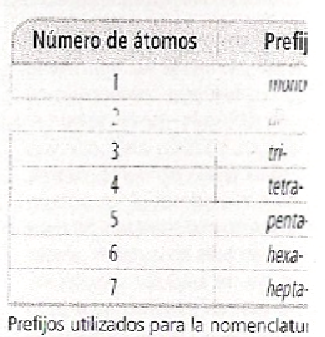

sistemática. Cl orefijo mono- suele on

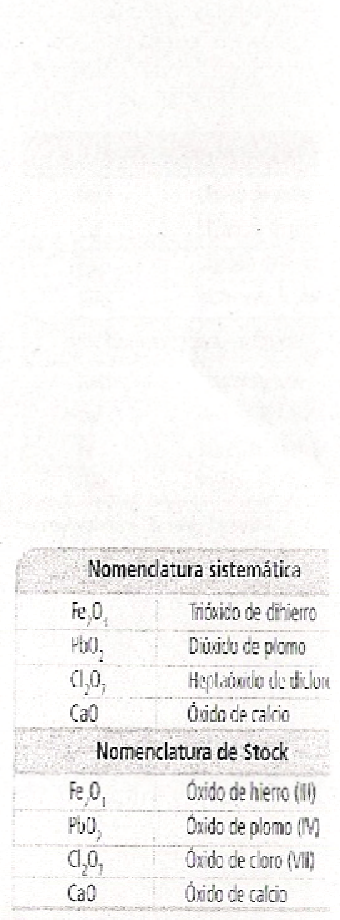

Nomenclatura de b́xidos

\section{ACTIVIDADES}

(2) Escribe el nombre o la fórmula, según sea el caso, de los siguientes óxidos:
a) $\mathrm{SiO}_{2}$.
b) $\mathrm{Ag}_{2} \mathrm{O}$
c) Trióxido de dialuminio
d) $\mathrm{Cl}_{2} \mathrm{O}_{5}$.
e) $\mathrm{BeO}$.
f) Óxido de antimonio (V)
g) $\mathrm{Ni}_{2} \mathrm{O}_{3}$.
h) $\mathrm{K}_{2} \mathrm{O}$.
i) Trioxido de azufre 


\begin{tabular}{|c|c|}
\hline \multicolumn{2}{|c|}{ Nomenclatura sistemática } \\
\hline $\mathrm{FeH}_{3}$ & Trihiduro de hiarro \\
\hline $\mathrm{PbH}_{4}$ & Tetrahidrum de plomo \\
\hline Nah & Hidruro de sotio \\
\hline $\mathrm{CH}$ & Mhidumdentrin \\
\hline \multicolumn{2}{|c|}{ Nomenclatura de Stock } \\
\hline $\mathrm{FeH}_{3}$ & Hidruro de hierro (in) \\
\hline $\mathrm{PbH}_{4}$ & Hiduro de plomo (M) \\
\hline $\mathrm{NaH}$ & Hidnuro de sodio \\
\hline $\mathrm{CaH}_{2}$ & Hidturo de ralio \\
\hline
\end{tabular}

Nomenclatura de hidruros metälicos.

\section{Otros hidruros no metálicos}

Cuando el hidrógeno se combina con elementos no metálicos de los grupos 14 y 15, forma hidruros que suelen identificarse mediante su nombre tradicional.

\begin{tabular}{|c|l|}
\hline Fórmula & Nombre \\
\hline $\mathrm{NH}_{J}$ & Amoniaco \\
\hline $\mathrm{PH}_{3}$ & Fosfina \\
\hline $\mathrm{CH}_{2}$ & Hetano \\
\hline $\mathrm{SH}_{2}$ & Silano \\
\hline
\end{tabular}

\section{Hidruros}

Los hidruros son compuestos binarios en los cuales uno de los elementos es el hidrógeno, que actúa con número de oxidación - 1 cuando se combina con un metal, y con +1 cuando lo hace con un elemento no metálico.

级

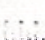

De acuerdo con la nomenclatura sistemática, se nombran de manera similar a los óxidos, pero empleando la palabra hidruro para indicar el tipo de compuesto. Lo mismo sucede con la nomenclatura de Stock:

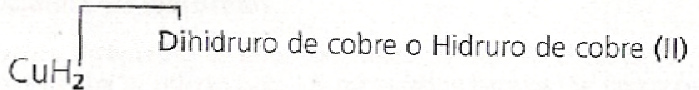

\section{Formulación de hidruros metálicos}

Para escribir la formula a partir del nombre, nuevamente tendremos en cuenta que los prefijos numerales nos indican el número de átomos de cada elemento en el compuesto. En el caso de la nomenclatura de Stock, el subindice del hidrógeno viene dado por el número de oxidación del elementn que se combina con él

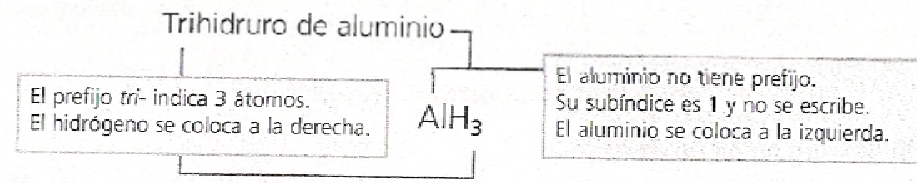

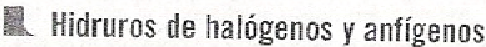

Al combinarse el hidrógeno con los elementos de los grupos 16 (anfígenos, exceptuando el oxígeno) y 17 (halógenos), resulta un grupo de compuestos denominados tradicionalmente ácidos hidrácidos. Para ellos se usan dos nomenclaturas: la sistemática, que indca el elemento no metálico con el sufijo -uro, y la tradicional, que usa el sufijo -hidrico.

\begin{tabular}{|c|c|c|}
\hline Fórmula & Nomenclatura sistemática & Nomenclatura tradicional \\
\hline $\mathrm{HF}$ & Fluouro de hidrogeneno & Ácido fluorhidico \\
\hline $\mathrm{HOI}$ & Cloruro de hidrógeno & Ácido clorhidrico \\
\hline $\mathrm{H}_{2} \mathrm{~S}$ & Sulfuro de dihidrógeno & Acito sulfidico \\
\hline
\end{tabular}

\section{ACTIVIDADES}

Escribe el nombre o la fórmula, según sea el caso, de los siguientes hidruros:
a) $\mathrm{NiH}_{2}$.
b) $\mathrm{ZnH}_{2}$
c) Hidruro de cadmio
d) $\mathrm{AgH}$.
e) $\mathrm{KH}$.
f) Dihidruro de estaño.
g) $\mathrm{CoH}_{3}$.
h) $\mathrm{MgH}_{2}$. 


\section{Sales binarias}

Las sales binarias son compuestos formados por la combinación de un elemento metálico con uno no metálico, distinto del hidrógeno y el oxigeno.

\section{望 Nomenclatura de sales binarias}

La nomenclatura sistemática de las sales binarias uúiza los prefijos numerales para indicar el número de átomos de cada elemento en la fórmula y el sufio -uro para el nombre del no metal. La nomenclatura de Stock usa la mismá téminación, sustituyendo los prefijos numerales por el número de oxidación del metal entre paréntesis:

$$
\mathrm{Fe}_{2} \mathrm{~S}_{3}{ }_{\text {Trisulfuro de dihierro o Sulfuro de hierro (III) }}
$$

\section{䐸 Formulación de sales binarias}

Para obtener la fórmula a partir del nombre se sique el mismo procedimiento que en el caso do ho oxidos e hidruros. Hay que tener en cuenta que aqui el metal se escribe a la izcuierda y el no metal a la derecha:

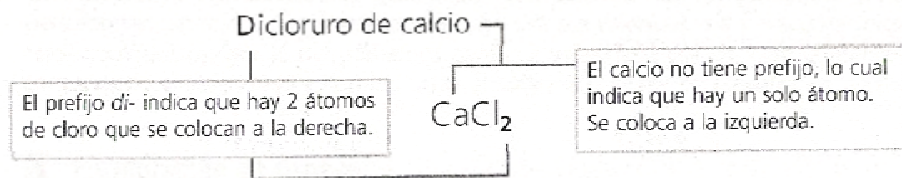

\section{ACTIVIDADES}

4. Escribe el nombre o la fórmula, según sea el caso, de las siguientes sales binarias:
a) $\mathrm{NiF}_{2}$.
b) $\mathrm{Ca}_{3} \mathrm{~N}_{2}$
c) Tric oruro de hierro.
d) $\mathrm{AgCl}$.
e) $\mathrm{KI}$.
f) Bromuro de sodio
g) $\mathrm{Cr}_{3}$
h) $\mathrm{Na}, \mathrm{Se}$
i) Seleniuro de plomo (IV)
j) CuS.
k) $\mathrm{Ca}_{2} \mathrm{C}$.
Sulfuro de dipótasio.
m) $\mathrm{BaBr}_{2}$
n) ZnS.
o) Fluoruro de magnesio.

Copia en tu cuaderno esta tabla y complétala con los nombres o fórmulas que faltan

\begin{tabular}{|c|c|c|}
\hline Fórmula & Nomenclatura sistemática & Nomenclatura de Stock \\
\hline \multicolumn{3}{|l|}{$\mathrm{P}, \mathrm{O}_{3}$} \\
\hline & & Oxido de azule (iV) \\
\hline & Óiszo de bario & \\
\hline & Dihiduro de berilio & \\
\hline \multicolumn{3}{|l|}{ LHH } \\
\hline & Dhidura de estronio & \\
\hline \multirow[t]{3}{*}{$\mathrm{Na}_{2} \mathrm{~S}$} & & \\
\hline & & Clour ode chtan (lli) \\
\hline & Bromito de mercurio & 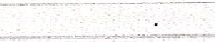 \\
\hline
\end{tabular}

Una sal muy scomún

Aunque normalmente asocia el términus sal al cloruro de s o sal común) que utilizarno: oulmico, esla padabid englob: amplio grupo de compuestos mados por la combinación de mentos metáliros con otros no tálicos, muchus de ellos de asp crisiditino y solubles en agua, bido a las uniones íninic as qu establecen entré los átomos kx insman.

\begin{tabular}{|c|c|}
\hline \multicolumn{2}{|c|}{ Nomenclatura sistemática } \\
\hline $\mathrm{MaCl}$ & Coruro de sodio \\
\hline $\mathrm{Car}_{\text {, }}$ & Difluoruro de wh \\
\hline Fel, & Diguduo de hien \\
\hline $\mathrm{ABl}_{3}$ & Tribromuro de all. \\
\hline \multicolumn{2}{|c|}{ Nomenclatura de Stock } \\
\hline $\mathrm{HaCl}$ & Clonuro de sodio \\
\hline $\mathrm{CaF}_{2}$ & Fiuoruro de calo: \\
\hline Fel, & Yoduro de hierio \\
\hline$A B r_{i}$ & Bromum de alum \\
\hline
\end{tabular}

Norendatura de sales hinarios 
¡Atención!

En un hidróxido, el meld se combina con tantos. grupos OH como

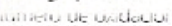

$\mathrm{Al}(\mathrm{OH})_{3}$

El número de oxidación del $\mathrm{A}$ es 13.
Hidróxidos

Los hidróxidos son compuestos ternarios en los cuales un metal se combina con uno varios grupos, $\mathrm{OH}^{-}$, formados por la unión de un átomo de oxigeno y uno de hidróge no. Estos grupos, que ticnen entidad química propia, reciben el nombre de hidroxilos u oxhidrilos.

Para nombrar y formular los hidroxidos los grupos $\mathrm{OH}^{-}$que poseen se tratan como una unidad independiente que actúa con número de oxidación -1 . Si hay varios, se colocan en la fórmula entre paréntesis y con el subindice correspondiente.

\section{凰 Nomenclatura de hidróxidos}

La nomenclatura sistemática de estos compuestos se basa en el uso de los mismos prefijos numerales que se utilizan para los compuestos binarios. Se nombran con la palabra hidróxido precedida del prefijo correspondiente al número de grupos $\mathrm{OH}^{-}$, sequida rel nombre dcl metal:

$$
\mathrm{Fe}(\mathrm{OH})_{2} \text { Dihidróxido de hierro }
$$

En la nomenclatura de Stock, se usa la palabra hidróxido y se indica el número de oxidación del metal entre paréntesis de la formu habitual, siempre que tenga más de uno posibie:

$$
\mathrm{Fe}(\mathrm{OH})_{2} \rightarrow \text { Hidróxido de hierro (II) }
$$

\section{Formulación de hidróxidos}

Para escribir la fórmula a partir del nombre, comenzamos ponienco el símbolo del metal a la izquierda y colocamos a la derecha el número de yrupos hidroxilo que indique el prefijo numeral, si el nombre corresponde a la nomenclatura sistemática. Si se trata de la nomenclatura de Stnrk, nl númcro de grupos $\mathrm{OH}^{-}$coincide con el número

\begin{tabular}{|c|c|c|}
\hline $\begin{array}{l}\text { El prefijo tetra- indica que hay } 4 \text { grupos } \\
\text { hidroxilo, que se colocan a la derecha. }\end{array}$ & $\mathrm{Pb}(\mathrm{OH})_{4}$ & $\begin{array}{l}\text { El plomo no tiene mriijo. } \\
\text { Hay un solo átomo que } \\
\text { se coloca a la izquierda. }\end{array}$ \\
\hline
\end{tabular}
de oxidación que va entre paréntesis.

\section{ACTIVIDADES}

6. Escribe cl nombre o la fórmula, según sea el caso, de los siguientes hidróxidos:
a) $\mathrm{Ni}(\mathrm{OH})_{2}$.
b) $\mathrm{Fe}(\mathrm{OH})_{3}$
c) Hidróxido de mercurio (III)
d) $\mathrm{Zn}(\mathrm{OH})$
e) $\mathrm{LiOH}$
f) Trihidróxido de aluminio
g) $\mathrm{AgOH}$
h) $\mathrm{Mg}(\mathrm{OH})_{2}$. 


\section{Oxoácidos}

Los oxoácidos son compugstos terriulus formados por hidrógeno, oxigeno y otro elemento, que suele ser un no metal, o un metal do liarlsición que actúa con su número de oxidación más alto. Se denominan así porque se trata de sustancias que poseen propiedades ácidas.

an monteiciatura uaticional de oxoáciuos

Para nombrar los oxoácidos se utilizan dos nomenclaturas; la sistemática y, priricipalmenle, la tradicional.

La nomenclatura tradicional os la mús utilizada para este tipo de compuestos. Se basa en el empleo de una serie de prefijos y sufijos, que se asignan de acuerdo con el número de oxidación con que actúe el no metal de entro todos lus yue posee:

\begin{tabular}{|c|c|c|c|c|c|}
\hline Prefijo & Sufilo & 1 valencia & 2 valencias & 3 valencias & 4 valencias: \\
\hline hipo- & 050 & & & Aodo hiponituoso. HNo & Ácido hiporloroso $\mathrm{HClO}$ \\
\hline- & $-0,0$ & & Acido sulfuroso: $\mathrm{H}_{2} \mathrm{SO}_{3}$ & Nido nitrom:HNO, & Áido doross $\mathrm{HCO}$ \\
\hline - & $-i c 0$ & Mido carbonica $\mathrm{H}_{2} \mathrm{CO}_{3}$ & Acido sulfirion $\mathrm{H}_{2} \mathrm{OO}_{4}$ & Audo nituico. HWO, & Acido clórico: $\mathrm{HCO}_{3}$ \\
\hline per- & -100 & & & & Acido pexlórico $\mathrm{HCO}_{4}$ \\
\hline
\end{tabular}

Observa que se consideran solo los estados do uxidación que los elementos utilizan para formar oxoácidos. Por ejemplo, el azıfre usa sus valoncius $+4 y+6$ para formar estos compuestos, por lo que debemos contar 2 valencias nociblos. Con la menor conresponde el sutijo -oso y con la mavor el sufijo -ico, como se muestra en la tabla.

\section{骺 Formulación de oxoácidos}

Hay varias formas de obtener la fórmula de un oxoácido. La más sencilla es formular el oxido y anadirla agua, pura lu cual necesitamos conocer el número dẹ oxidación con que actúa el elemento que da lugar al compucsto cicido. Observa este ejemplo:

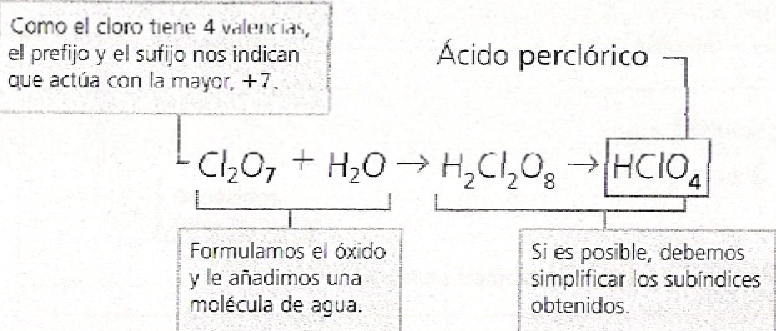

\section{ACTIVIDADES}

7. Escribe el numbre o la formula, según sea el caso, de los siguientes oxoácldos:
d) $\mathrm{H}_{3} \mathrm{PO}_{4}$.
b) $\mathrm{H}_{2} \mathrm{SiO}_{3^{\prime}}$
c) Ácido brómico.
d) $\mathrm{HBrO}$
e) $\mathrm{HIO}_{2}$
f) Árido peryodico
g) $\mathrm{H}_{3} \mathrm{BO}_{3}$.
h) $\mathrm{HSbO}_{2}$.
i) Ácido selenioso

Nomenclatura sistemáti de los ácidos

La nomenclatura sistemát los ácidos es algo más col nue la nnmenrlatura tradi Un prefjo murneral, seguic término oxo, indica el núm átomos de oxígeno, seguil combire del elemento centr minado en ralo, con su va entre parentesis. Si hay $n$ un álomo de este elemen antepone el prefiio nu correspondiente. Finalizan mumbre añadiendo las pa de hidrógeno:

$$
\mathrm{H}_{2} \mathrm{Cr}_{2} \mathrm{O}_{7}
$$

Heptaoxodicromato ( de hidrógeno

$$
\mathrm{H}_{2} \mathrm{CO}_{3}
$$

Irioxocarbonato (IV) de hidrugeno

\section{Hay excepcione}

Detorminados ácicos —com del fósforo- se forman an do más de una molécula de al óxido. En estos casos se usa prefinos $m$ wi, fim y orto hacer referencia a los ácidos ridos con una, dos o tres mo lis dis aulut, rumetivemente 


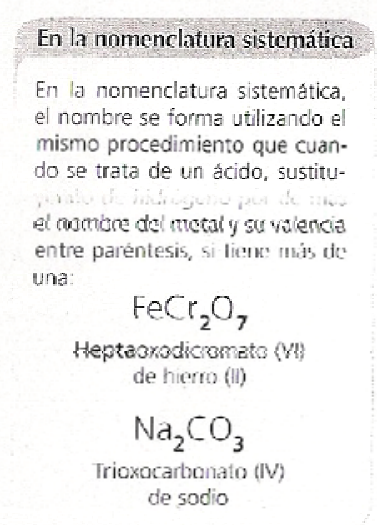

\begin{tabular}{|c|c|}
\hline \multicolumn{2}{|r|}{ Nomenclatura tradicional } \\
\hline $\mathrm{FeCO}_{3}$ & Carbonato de hierno (it) o forroso \\
\hline $\mathrm{NaClO}$ & Hipoulorito de sodio \\
\hline $\mathrm{AlPO}_{4}$ & Fosfato de aluminio \\
\hline$\left(a\left(\mathrm{NO}_{3}\right)_{2}\right.$ & Nitrato de calcio \\
\hline
\end{tabular}

\section{Sales ternarias u oxisales}

Las sales ternarias u oxisales están formadas por oxígeno y dos elementos - un meta y un no metal o metal de transición-. Se pueden considerar compuestus derivados de los oxodcidos, en los que se ha sustituido el o los hidrógenos por un metal. Por lo tanto. su nomenclatura es lá relacionada con la de los ácidos de los que proceden.

Según la nomenclatura tradicional, las soles se nombran sustituyendo los sufijos -oso

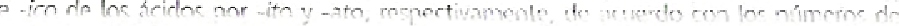
oxidacion del elemento central.

\begin{tabular}{|c|c|c|c|c|c|c|c|}
\hline Tlene 1 valencia & \multirow{2}{*}{ Tiene 2 valencias } & \multicolumn{3}{|c|}{ Tiene 3 valencias } & \multicolumn{3}{|c|}{ Tiene 4 valencias } \\
\hline - & & hipo- & $\ldots$ & ito & hiog. & $\ldots$ & tho \\
\hline- & -10 & & & tito & & & tito \\
\hline ato & atto & & & $-2 t 0$ & & & dto \\
\hline- & - & & & - & per- & $\ldots$ & vito \\
\hline
\end{tabular}

El nombre del metal que sustituye a los átomós de hidrógeno va después de la preposición de y su número de oxidación se coloca entre paréntesis, si puede tener más de uno. Observa este ejemplo

$$
\underset{\text { Ácido sulfúrico }}{\mathrm{H}_{2} \mathrm{SO}_{4}} \rightarrow \begin{gathered}
\mathrm{PbSO}_{4} \\
\text { Sulfato de plomo (II) }
\end{gathered}
$$

Otra posibilidad frecuente es usar los mismos sufijos (-oso e -ico) para indicar el estado de oxidación del metal:

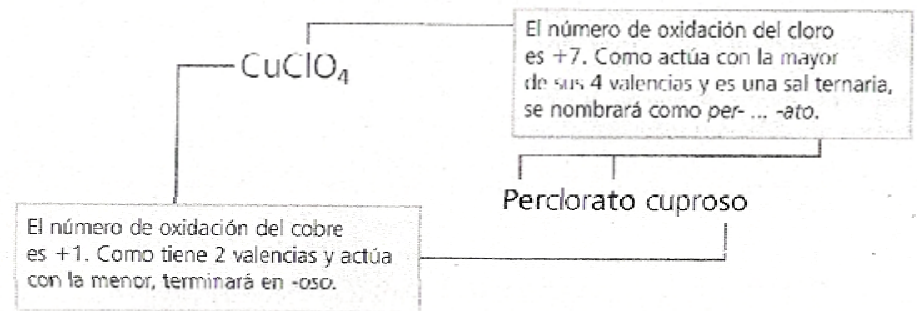

Observa que el metal que sustituye ul hidrógeno se escribe a la izquierda, mientras que el grupo formado por el no metal y el oxigeno - que es un verdadero anión con entidad química propia- aparece a la derecha en la fórmula.

\section{ACTIVIDADES}

65. Escribe el nombre de las siguientes sales
a) $\mathrm{Li}_{2} \mathrm{SO}_{4}$
b) $\mathrm{FePO}_{4}$
d) $\mathrm{KClO}_{2}$.
e) $\mathrm{CaCO}_{3}$
c) $\mathrm{NaIO}_{4}$.
g) $\mathrm{NaNO}_{2}$
h) $\mathrm{NiSO}_{3}$.
CuBrO
i) $\mathrm{AlPO}_{3}$

5i De cada grupo de las siguientes fórmulas hày una que no es correcta. Indica de cuál se trata y justifica tu elección en cada caso:

d) Ácidos: $\mathrm{HClO}_{7} ; \mathrm{H}_{2} \mathrm{Cr}_{2} \mathrm{O}_{7} ; \mathrm{HSO}_{4} \mathrm{H}_{3} \mathrm{PO}_{3}$

b) Oxisales: $\mathrm{CaSlO}_{3} ; \mathrm{MgCr}_{2} \mathrm{O}_{7} ; \mathrm{FeSO}_{3} ; \mathrm{Na}_{2} \mathrm{ClO}_{3}$. 


\section{De la fórmula al nombre paso a paso}

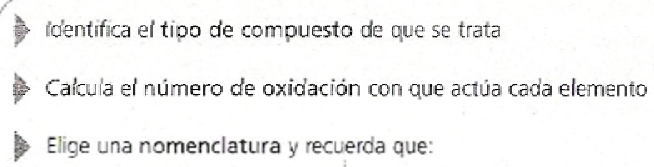

$\rightarrow$ Nomenclatura tradicional: prefijos y sufijos según el número de oxidación utilizado

5ivi Indica para cada compuesto el nombre o los nombres que le corresponden según las nomenclaturas estudiadas en el esquema anterior:
a) $\mathrm{Sn}(\mathrm{OH})_{2}$
b) $\mathrm{NaH}$.
c) $\mathrm{NaBr}$.
d) $\mathrm{HClO}_{4}$.
e) $\mathrm{Hg}_{2} \mathrm{SO}_{4}$.
f) $\mathrm{SO}_{2}$.
h) $\mathrm{MgCO}_{3}$
i) $\mathrm{Ca}(\mathrm{OH})_{2}$
j) $\mathrm{FeCl}_{2}$.
k) $\mathrm{Na}_{2} \mathrm{O}$
l) $\mathrm{HNO}_{3}$.
m) $\mathrm{Ca}_{3} \mathrm{P}_{2}$.
n) $\mathrm{Au}_{2} \mathrm{O}_{3}$.
ก) $\mathrm{Pb}(\mathrm{OH})_{2}$.
o) $\mathrm{COSO}_{3}$.
q) $\mathrm{FeH}_{3}$.
r) $\mathrm{CuClO}_{3}$.
5) $\mathrm{Al}_{2} \mathrm{~S}_{3}$.
t) $\mathrm{H}_{2} \mathrm{SeO}_{4}$
u) $\mathrm{Cl}_{2} \mathrm{O}$.
v) Cul.
x) $\mathrm{Ni}(\mathrm{OH})_{3}$.
y) $\mathrm{PbO}_{2}$. 
ANEXO IV: Vidal, M.C., de la Prada, F., de Luis, J.L. (2007). Física y Química 3º ESO. Madrid: Santillana

\section{ANEXO IV}

\section{Formulación}

\section{ACTIVIDADES}

1. Ee Escribe la contiguración electrónica de los siguięntes: elementos:
a) $\mathrm{He}(Z=2)$. d) $\mathrm{Mg}(Z=12)$
b) $\mathrm{O}(Z=8)$. e) $\mathrm{Cl}(Z=17)$
c) $\mathrm{F}(Z=9)$. i) $\operatorname{Ar}(Z=18)$.

2. Eseribe el ion que formarán lọs siguientes atomos:
a) $\mathrm{Li}$
d) Al
b) $F$
e) $\mathrm{CI}$
c) $\mathrm{Na}$
f) $\mathrm{k}$

\section{Configuración electrónica de los elementos}

En este libro hemos visto las experiencias que llevaron a escablecer los mo delos átómicos. Bohr diseñó un modelo segùn el cual los electrones se encuentran en determinados niveles alrededor del nụcleo del a tomo; cstos niveles tienen distinta energia, por eso se denominan niveles energetricos. El

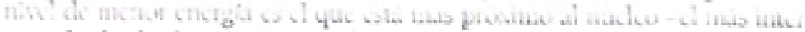
no-; desde ahi, la energia aumenta hasta liegar al más exterior.

En la coneza de lós átomos pueden existir hasta siete niveles energeticos que se desiguan con un número o una letra segun su proximidad al núleo. Los electrones se colocan en los niveles en orden creciente de energia y si. guiendo ciertas normas

- El nivel más interno solo puede albergar 2 elecrromes.

- El scgundo nivel puede albergar, como maximo, 8 electrones.

- El tercer nivel puede albergar, como máximo, 18 electrones; pero cuando este llega a tener 8 electrones, comienza a llenarse el cuartó

Pot ejemplo, elátomo de pobasio tiene 19 eloctrones que se colocarán en los niveles del modo siguiente:

$$
\begin{aligned}
& \text { Nivel } 1 \rightarrow 2 \text { electrones } \\
& \text { Nivel } 2 \rightarrow 8 \text { electrones } \\
& \text { Nivel } 3 \rightarrow 8 \text { electrones } \\
& \text { Nivel } 4 \rightarrow 1 \text { electrón }
\end{aligned}
$$

Se llama configuración electróniça de un elemento al modo en que se organizan los electrónes en un átomo dé esse elemento.

La configuración electrónica del potasio es; $2.8,8,1$.

El ưltimo nivel en la que un atomo tiene electrones se llama nivel de valencia. El K tiene 1 electrón en su nivel de valencia.

Tódos lós elementos del grupo 18 tienen 8 electrones en su ûltimo nivel, con la excepción del He, qué solo tiene 2. Los elementos de este grupo se llaman gises nobles porque son muy estables y no se combinan con ningun otro elemento.

Los demás elementos de la tabla periodica tratarain de alcanzar la configura. ción de los gases nobles, porque asi aumentara su estabilidad. Para ello, ga. narin o perderín los clectrones que sean necesarios y dejarin de ser cspe. cies neutras; se convertiran en jones.

Un ion es un átomo que ha ganado o perdido electrones.

* Cuando un átomo pierde electroncs, adquiere carga positiva y se conviertè en un ion positivo o catión

Por ejemplo, el cloro (Cl) tiene en su úlimo nivel la configuracion: 2,7 para, conseguir la configuración de gas noble se convierte en el ion Cl-,

- Cuando un atomo gana electrones, adquiere carga negativa y se convierte en un lon négativo o aniòn

Por ejemplo, d soxtio (Na) thene en su ultimo nivel la configuración: 1, y part conseguir la configuración de gas noble se convierte en cl ion $\mathrm{Na}^{*}$. 


\section{Formulación inorgánica}

Del mismo modo que utilizamos las palabras para comunicamos, en quimica recurrimos a las fórmulas parà representar los compuestos. Estas fómulas no son arbitrarias, sino que responden a unas reglas establecidas, reglas que han variado a lo largo del tiempo, desde la nomenclatura tradicional, pasando por

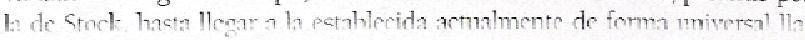
mada sistemática o IUPAC (Unión Internacional de Química Pura y Aplicada). Antes de aprender a formular debemos estudiar lós conceptos dé valencia y número de oxidación:

\section{Valencia}

Es la capacidad que posee un elemento para combinarse con otro. El átomo de hidrógeno sé toma como referencia, ya que tiene valencia 1 . Así se define nuevamente la valencia como el número de átomos de hidrógeno que se puede combinar con un átomo de cualquier otro elemento.

\section{Número de oxidación o de valencia}

Es el número de electrones que un átomo puede captar o ceder (cotal o parcialmente) al formar un compuesto. Es negativo si gana electrones y positivo si los pierde.

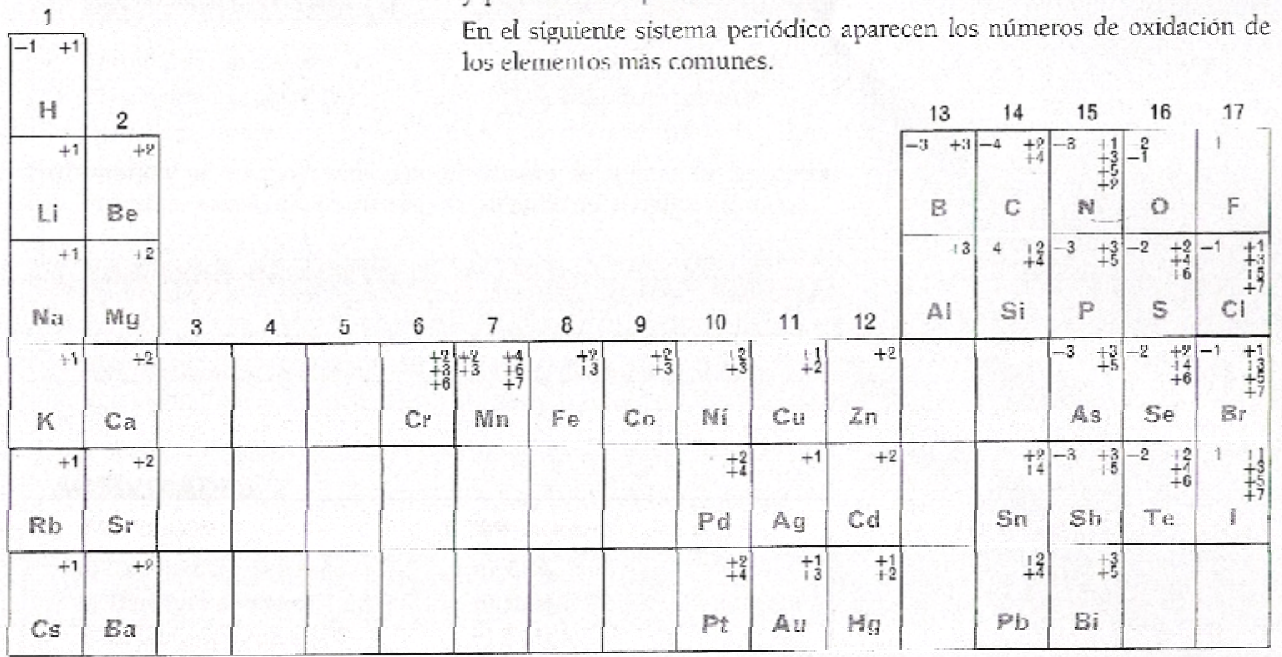

\section{EJERCICIO RESUELTO}

Clorura dé sodio: $\mathrm{NaCl}$ Fluoruro de hidrógeno: HF Sulfuro de hidrógend: $\mathrm{H}_{2}$ Sे Sulfato de cobre (II): CuSO
Como regla general:

En cualquier fórmula química se escriben en primer lugar los elementos situados a la izquierda del sistema periódico estos elementos tienen tendencia a formar iones positivos o cationes, y en segundo lugar los situados a la derecha que son elementos que tienen tendencia a formar iones negativos o aniones.

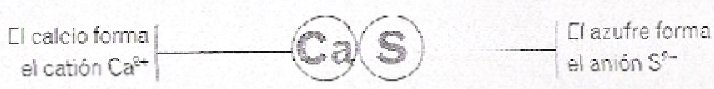


PEROXIDOS

Los peróxidos sor

las combinaciones del grupo peroxo $\mathrm{O}_{2}^{2-}$ con metales

ocon hidiógenos.

El ejemplo máts común

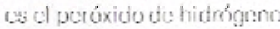

$\left(\mathrm{H}_{2} \mathrm{O}_{2}\right)$ conocido como agua

oxigenada.

\section{Compuestos binarios con oxígeno}

\section{1 óxidos}

Los oxidos son las combinaciones del oxigeno con otro elentento, meial o no metal. En estos compuestos el oxigeno siempre actúa con número de oxidación -2 .

Se formulan anteponiendo al simbolo del oxigeno el del elemento (metal o no metal) e intercambiando las valencias, que se colocan como subíndices, y simplificando siempre que sea posible.

\section{EJERCICIO RESUELTO}

- $\mathrm{Pb}_{2} \mathrm{O}_{2} \rightarrow \mathrm{PbO}_{2}$

- $\mathrm{Ca}_{9} \mathrm{O}_{2} \rightarrow \mathrm{CaO}$

\section{Nomenclatura}

Stock: se nombran con la palabra oxido seguida del nombre del metá o nó metal e indicando entre paréntesis el numero de oxidación, en numcros romanos, solo en caso de tener más de un número.

\section{EJERCICIO RESUELTO}

- $\mathrm{Cl}_{2} \mathrm{O}$ : óxido de clora (V)

- $\mathrm{PbO}_{2}:$ óxido de plomo (IV)

Sistemática: se nombran ndicando el numero de átomos de cada elemento con los prefijos mono-, di-, tri-, tetra-, etc,

\section{EJERCICIO RESUELTO}

- CaO: monóxido de monocalcio.

Cuando no cause confusion, se suprime el pretijo mono-

en este caso sería correcto decir óxido de calcio.

- Clo pentíxicio rde diclom.

\section{ACTIVIDADES}

\section{3. $\rightarrow$ Formula:}

a) Óxido de plorno (II).

4. 불 Nombra:

b) Monoxido de cromo.

a) $\mathrm{Cr}_{2} \mathrm{O}_{3}$

c) Monoxido de cobre.

b) $\mathrm{Ha}_{2} \mathrm{O}$

d) Óxido de platino (IV)

c) $\mathrm{CrO}$

e) Óxido de estaño (1)

d) $\mathrm{ZnO}$

f) Monóxido de estaño.

e) Pto

g) Óxido de mercurio (1).

f) $\mathrm{Ni}_{2} \mathrm{O}_{3}$

h) Óxido de plata (I)

g) $\mathrm{SnO}_{2}$

i) Dióxido de carbono.

i) $\mathrm{SiO}_{2}$ 


\section{Compuestos binarios con el hidrógeno}

\subsection{Hidruros metálicos}

Los hidruros melálicos son las combinaciones del hidrógeno con un mettal. En estos compuestos el hidrógeno siernpre aclúa con nümero de oxidación -1.

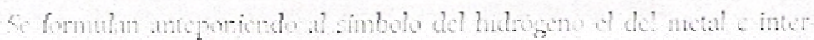
cambiandu lis valencars, que se colocan como subindices.

\section{EJERCICIO RESUELTO}
- $\mathrm{CaH}_{2}$
- $\Gamma$ th
- $\mathrm{PbH}_{4}$
- $\mathrm{CuH}_{2}$

\section{Nomenclatura}

Stock: se nombran con la palabra hidruro seguida del nombre del metal c indicando entre parénlesis el numem de oxidacion, en numeros romanos, solo en caso de tener más de un número de oxidación

\section{EJERCICIO RESUELTO}
- Call ${ }_{2}$ : hidruro de calcio
- Pethis hidruro de olatino (V)
- Hhil hidrurn de plomo (IV)
- $\mathrm{CuH}_{2}$ : hidruro de cobrc (II).

Sistemática: se nombran indicando el número de attomos de hidrogeno

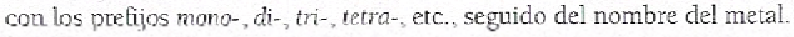

\section{EJERCICIO RESUELTO}
- Catb: dihidruro de calcio
- $\mathrm{PHH}_{4}$ : tetrahidruro de platino.
- PbH: tetrahidiruro de plomo
- $\mathrm{CuH}_{2}$ : dihidrura de cobre

\section{ACTIYIDADES}

\section{Formula}
a) Tetrahidruro de estaño.
b) Dihidrurn de hierro
c) Dihidruro de cobre.
d) Hidruro de hierro (III).
e) Hidruro de plomo (II).
f) Hidruro de calcio.
g) Hidruro de sodio.
h) Trihidruro de oro
D) Dihidruro de plata.
j) Trihidruro de niquel.
k) Hidruro de cobre.

6. 9 Nombra:
a) $\mathrm{CiH}_{3}$
b) $\mathrm{CaH}_{2}$
c) $\mathrm{CuH}_{2}$
d) $\mathrm{CuH}$
e) $\mathrm{LiH}$
f) $\mathrm{CrH}_{0}$
g) $\mathrm{AgH}_{2}$
h) $\mathrm{LiH}$
i) $\mathrm{MgH}_{2}$
j) $\mathrm{FeH}_{2}$
k) $\mathrm{ZnH}_{2}$ 


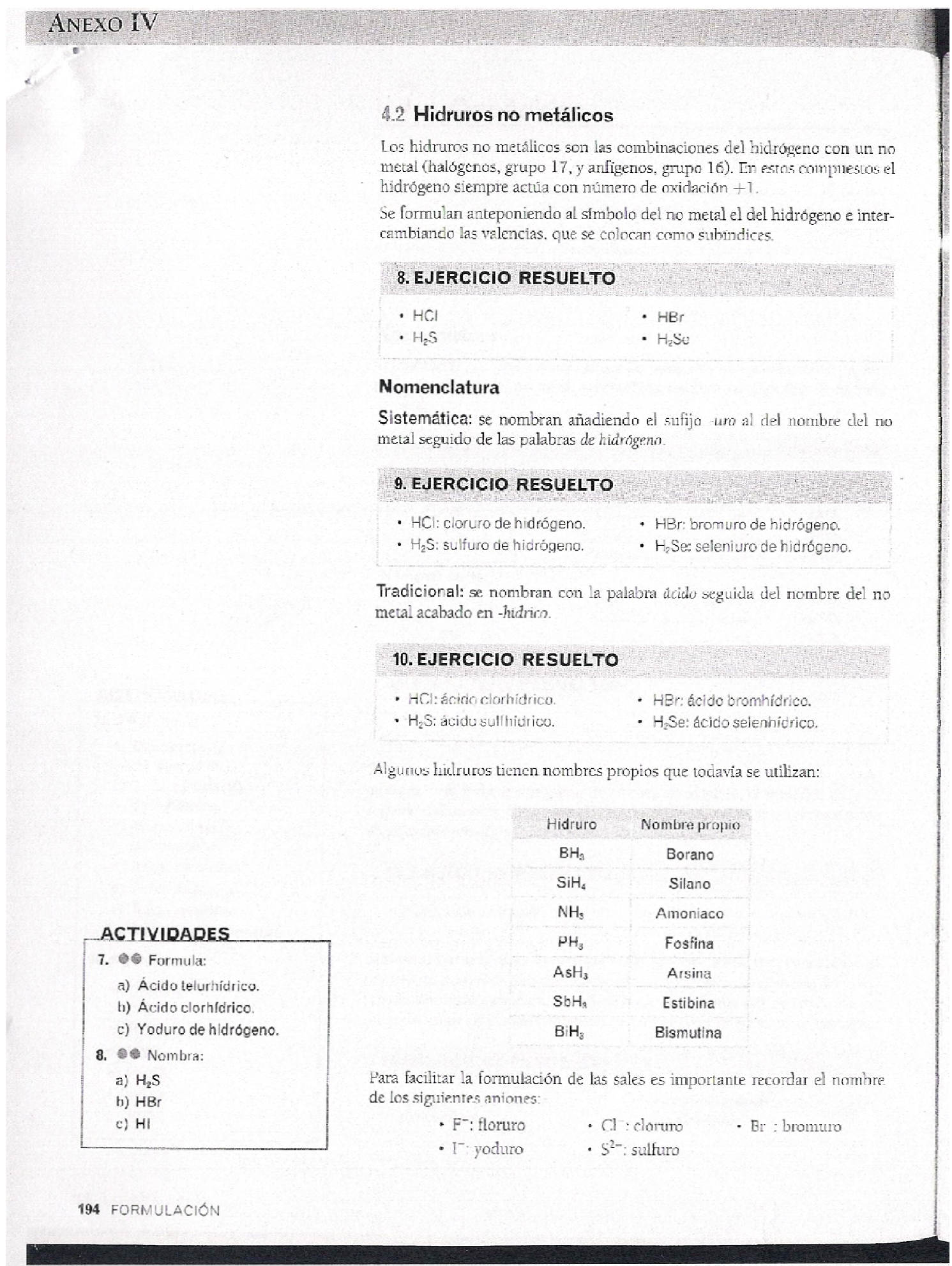




\section{Sales y otros compuestos binarios}

Las sales binarias son la combinación de no metales con un no metal. Si se combinan dos no metales, se considera el positivo aquel que está más a la izquierda en la siguiente lista:

$$
\mathrm{B}, \mathrm{Si}_{1}, \mathrm{C}, \mathrm{Sh}, \mathrm{A}, \mathrm{P}, \mathrm{N}, \mathrm{H}, \mathrm{Te}, \mathrm{Se}, \mathrm{S}, \mathrm{At}, \mathrm{I}, \mathrm{Br}, \mathrm{Cl}, \mathrm{O}, \mathrm{s}
$$

\section{Nomenclatura}

Stock: para nombrar el compuesto se añade el sufijo -uro al nombre del elemento situado a la derecha, seguido del nombre del elemento que aparece a la izquierda e indicando su número de oxidación entre paréntesis y en números romanos, solo en el caso de que tenga más de un número de oxidación.

\section{EJERCICIO RESUELTO}

- $\mathrm{PCl}_{5}$ : cloruro de fóstoro (V). - $\mathrm{FeBr}_{3}$ : bromuro de hierro (iil).

Sistemática: se nombran indicando el número de átomos de cáda elemento con los prefijos mono-, di-, tri-, tetra-, etc, y añadiendo el sufijo -uro al nombre del elemento situado a la derechâ de la formula.

\section{EJERCICIO RESUELTO}

- PCl: pentacloruro de fósforo. - $\mathrm{FeBr}_{3}$ : tribromuro de hierro

\section{Hidróxidos}

Los hidróxidos son los compuestos que resultan de la combinación de los metales con el ion hidróxido $\left(\mathrm{OH}^{-}\right)$. Se formulan con el símbolo del metal seguido del grupo $\mathrm{OH}$ e intercambiando las valencias.

\section{Nomenclatura}

Stock: se antepone la pablabra hidróxido al nombre del metal, indicando su número de oxidación en números romanos y entre paréntesis.

\section{EJERCICIO RESUELTO}

- $\mathrm{NaOH}$ : hidróxido de sodio. $\quad$ Sn(OH) hidróxido de estaño (iV)

Sistemática: se nombran indicando el número de grupos $\mathrm{UH}$ con los prefijos mono-, di-, tri-, tetra-, etc., añadiendo a continuación el nombre del metal.

\section{EJERCICIO RESUELTO}

- NaOH: hidróxido de sodio. - Sn(OH) $\quad$ - tetrahidrớdido de estano.

\section{ACTIVIDADES}

9. $\odot$ Formula:

a) Cloruro de bario.

b) Tetrayoduro de estaño.

c) Cloruro de calcio.

d) Dicloruro de mercurio.

10. 9 농 Nombra:
a) $\mathrm{PbCl}_{2}$
b) $\mathrm{CsCl}$
c) $\mathrm{AIF}_{3}$
d) $\mathrm{Bal}_{2}$

ACTIVIDADES

11. 술 Formula:

a) Dihidróxido de cobre.

b) Dihidróxido de cinc.

c) Hidróxido de platino (II).

d) Hidróxido de cobre (II).

12. 5 e Nombra:
a) $\mathrm{Ni}(\mathrm{OH})_{2}$
b) $\mathrm{Al}(\mathrm{OH})_{3}$
c) $\mathrm{Fe}(\mathrm{OH})_{2}$
d) $\mathrm{CsOH}$ 


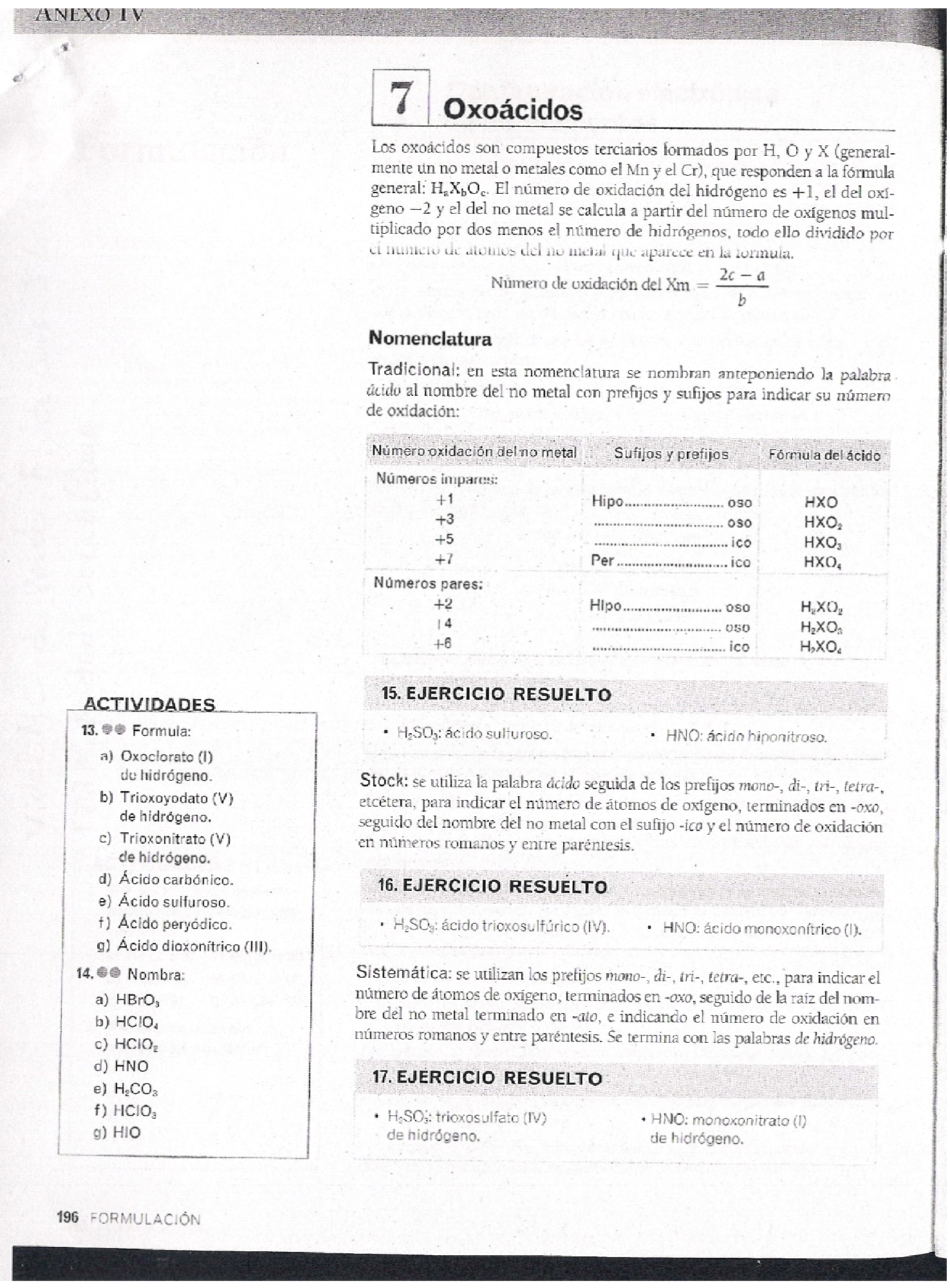




\section{Sales terciarias u oxisales}

Las seles terciarias u oxisales son los compuestos que resultan de cambiar los hidrogenos de los oxoácidos por metales

\section{Nomenclatura}

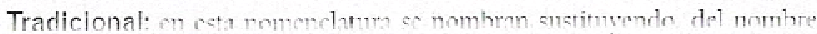
del no mesal $(X)$, los sufijus -oso e-ico por -ito y -ato.

\begin{tabular}{|c|c|c|c|}
\hline $\begin{array}{c}\text { Número oxidación } \\
\text { del no metál }\end{array}$ & $\begin{array}{l}\text { Sufijos y prefijos } \\
\text { en ácidos }\end{array}$ & $\begin{array}{c}\text { Sufijos y prefijos } \\
\text { en sales }\end{array}$ & $\begin{array}{c}\text { Anión procedente } \\
\text { del ácido }\end{array}$ \\
\hline \multicolumn{4}{|l|}{ Números impares: } \\
\hline+1 & Hipo .......... oso & Hipo............... ito & $\mathrm{XO}^{-}$ \\
\hline+3 & ................ oso & ..................... ito & $\mathrm{XO}_{2}^{-}$ \\
\hline+5 & ( & $\ldots$ & $\times 0_{3}^{-}$ \\
\hline+7 & Per............... ico & Per ............... ato & $\mathrm{XO}_{4}$ \\
\hline \multicolumn{4}{|l|}{ Números pares: } \\
\hline+2 & Hipo .......... OSO & Hipo n...ен... ito & $\left(\mathrm{XO}_{2}\right)^{2-}$ \\
\hline+4 & ………......... OSD & 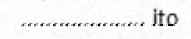 & $\left(\mathrm{XO}_{2}\right)^{2}$ \\
\hline+6 & ico & .................... ato & $\left(\mathrm{XO}_{4}\right)^{2}$ \\
\hline
\end{tabular}

\section{EJERCICIO RESUELTO}

- $\mathrm{ZnSO}_{3}$ : sulfito de cinc

- Cu(NO) $)_{2}$ nitrito de cobre (llt.

- FeSO: sultato de hierro (ll).

- $\mathrm{Pb}\left(\mathrm{NO}_{3}\right)_{4}$ nitrito de plorno (IV)

- $\mathrm{CaCO}_{3}$ : carburalo de calcio.

- AxClo: hipoclorito de plata.

Stock: se nombran igual que en la nomenclatura tradicional, pero se indica la valencia del metal entre paréntesis y con números romanos.

\section{EJERCICIO RESUELTO}

- $\mathrm{ZnSO}$ : sulfito de cinc.

- $\mathrm{Pb}\left(\mathrm{NO}_{2}\right)_{4}$ : nitrito de plomo ( $\left.\mathrm{W}\right)$.

- $\mathrm{Cu}\left(\mathrm{NO}_{2}\right)_{2}$ : nitrito de cobre (II).

- $\mathrm{CaCO}_{3}$ : carbonato de calcio.

- FeSO: sulfato de hierro (II)

Sistemática: se nombran igual que en los ácidos; solo se cambian las palabras de hidrógeno por el nombre del metal con la valencia entre paréntesis y con números romanos.

\section{EJERCICIO RESUELTO}

- $\mathrm{ZnSO}_{3}$ : trioxosulfato (IV) de cinc.

- $\mathrm{Cu}\left(\mathrm{NO}_{2}\right)_{2}$ : dioxonitrato (ili) de cobre (II).

- FeSO- tetraoxosulfato (VI) de hierro (II).

- AuNO: monoxonitrato (l) de oro (1).

- Cacos trioxocarbonato (IV) de calcio.

\section{SALES ACIDAS}

Cuando los oxoácidos tienen más de un hidrógeno pueden

formar aniones que conservan

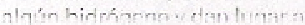

salesacidas

Por ejemplo:

$$
\mathrm{NaHCO}_{3}
$$

Bicarbonalo do sodio Monohidrógenocarbonato de sodio
ACTIVIDADES

\section{5. 룰 Furmula}

a) Hipoclorito de plata.

b) Yodato de niquel (II).

c) Carbonato de sodio.

d) Clorato de níquel (III).

e) Nitrato de cinc.

f) Trioxosultato (IV) de plomo (II).

g) Trioxonitrato (V) de plata.

16. *) Nombra:
a) $\mathrm{CoSO}_{3}$
b) $\mathrm{Al}_{2}\left(\mathrm{SO}_{4}\right)_{3}$
c) $\mathrm{ZnSO}_{3}$
d) $\mathrm{CaCO}_{3}$
e) $\mathrm{Pb}\left(\mathrm{NO}_{2}\right)_{4}$
f) $\mathrm{KClO}$
g) $\mathrm{Na}_{2} \mathrm{SO}_{4}$ 